\title{
Total Syntheses of Strasseriolide A and B, Antimalarial Macrolide Natural Products
}

Leah J. Salituro, Jessica E. Pazienza, and Scott D. Rychnovsky*

Department of Chemistry, University of California at Irvine, 1102 Natural Sciences II, Irvine, California 92697, United States

\section{Supporting Information}

$\underline{\text { Table of Contents }}$

Page

General Experimental

Synthesis of Aldehyde Coupling Partner 3

S3

Synthesis of Vinyl Iodide Coupling Partner 16

S12

Final Macrocyclization

References

S26

NMR Spectra

S27 


\section{General Experimental Details}

Chemical shifts $(\delta)$ were referenced to the residual solvent peak. The ${ }^{1} \mathrm{H}$ NMR spectra were recorded at $500 \mathrm{MHz}$ or $600 \mathrm{MHz}$ using either a Bruker DRX500 (cryoprobe) or a Bruker AVANCE600 (cryoprobe) NMR, respectively. The ${ }^{13} \mathrm{C}$ NMR spectra were recorded at $126 \mathrm{MHz}$ or $151 \mathrm{MHz}$ on the Bruker DRX500 or Bruker AVANCE600 NMR, respectively. All NMR spectra were taken at $25^{\circ} \mathrm{C}$. Chemical shifts $(\delta)$ are reported in parts per million (ppm) and referenced to residual solvent peak at $7.26 \mathrm{ppm}\left({ }^{1} \mathrm{H}\right)$ or $77.16 \mathrm{ppm}\left({ }^{13} \mathrm{C}\right)$ for deuterated chloroform $\left(\mathrm{CDCl}_{3}\right)$, and $3.31 \mathrm{ppm}\left({ }^{1} \mathrm{H}\right)$ or $49.15\left({ }^{13} \mathrm{C}\right)$ for deuterated methanol $\left(\mathrm{CD}_{3} \mathrm{OD}\right)$. The ${ }^{1} \mathrm{H}$ NMR spectral data are presented as follows: chemical shift, multiplicity $(s=$ singlet, $d=$ doublet, $t=$ triplet, $q=$ quartet, quint $=$ quintet, $\mathrm{m}=$ multiplet, $\mathrm{dd}=$ doublet of doublets, $\mathrm{ddd}=$ doublet of doublet of doublets, dddd $=$ doublet of doublet of doublet of doublets, $\mathrm{dt}=$ doublet of triplets, $\mathrm{dq}=$ doublet of quartets, $\mathrm{ddq}$ = doublet of doublet of quartets, app. = apparent), coupling constant(s) in hertz (Hz), and integration. High-resolution mass spectrometry was performed using ESI-TOF and Cl-TOF. An internal standard was used to calibrate the exact mass of each compound. For accuracy, the peak selected for comparison was that which most closely matched the ion intensity of the internal standard.

Unless otherwise stated, synthetic reactions were carried out under an atmosphere of argon. All commercially available reagents were used as received unless stated otherwise. Solvents were purchased as ACS grade or better and as HPLC-grade and passed through a solvent purification system equipped with activated alumina columns prior to use. $C D C l_{3}$ and $C D_{3} O D$ was purchased from Cambridge Isotope Laboratories. (R)-(+)-citronellic acid and $(R, S)$-Josiphos was purchased from Sigma Aldrich. (S)-(-)-citronellal was purchased from TCl Chemicals. Thin layer chromatography (TLC) was carried out using glass plates coated with a $250 \mu$ m layer of $60 \AA$ silica gel. TLC plates were visualized with a UV lamp at $254 \mathrm{~nm}$, or by staining with Hanessian's stain or $\mathrm{KMnO}_{4}$ stain. Liquid chromatography was performed using forced flow (flash chromatography) with an automated purification system on prepacked silica gel $\left(\mathrm{SiO}_{2}\right)$ columns unless otherwise stated. High-resolution mass spectra (HRMS) were recorded on Waters LCT Premier TOF spectrometer with electrospray ionization (ESI) and chemical ionization $(\mathrm{Cl})$ sources. Optical rotations were performed on a JACSO P-1010 spectrometer using a glass 10 $\mathrm{mm}$ cell with the sodium D-line at $589 \mathrm{~nm}$. 
Synthesis of Aldehyde Coupling Partner:

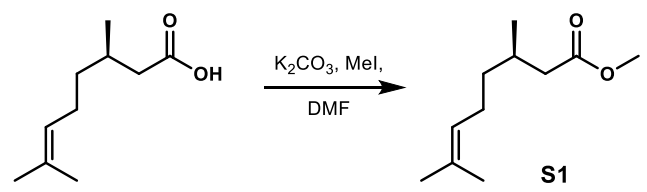

\section{Methyl (R)-3,7-dimethyloct-6-enoate (S1):}

To a solution of $(R)-(+)$-citronellic acid $(6)(4.0 \mathrm{~mL}, 21.7 \mathrm{mmol}, 1.0$ equiv) in DMF (43 mL) at rt was added $\mathrm{K}_{2} \mathrm{CO}_{3}(9.62 \mathrm{~g}, 69.9 \mathrm{mmol}, 3.2$ equiv), followed by iodomethane $(4.7 \mathrm{~mL}, 76.1 \mathrm{mmol}$, 3.5 equiv). After stirring at rt for $16 \mathrm{~h}$, the solution was diluted with $\mathrm{H}_{2} \mathrm{O}(100 \mathrm{~mL})$ and extracted with $\mathrm{Et}_{2} \mathrm{O}(5 \times 100 \mathrm{~mL})$. The combined organic phase was washed with brine, dried with $\mathrm{MgSO}_{4}$, filtered, and concentrated in vacuo. The resulting clear oil was subjected to the next step without further purification. The spectroscopic data matches that of which has been previously reported. ${ }^{1}$

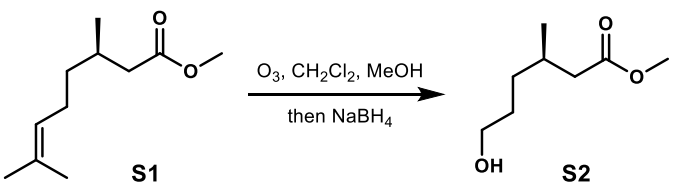

Methyl (R)-6-hydroxy-3-methylhexanoate (S2):

A solution of alkyne $\mathbf{S} 1\left(4.0 \mathrm{~g}, 21.7 \mathrm{mmol}, 1.0\right.$ equiv) in $\mathrm{CH}_{2} \mathrm{Cl}_{2}(80 \mathrm{~mL})$ and $\mathrm{MeOH}(20 \mathrm{~mL})$ at $-78{ }^{\circ} \mathrm{C}$ was treated with $\mathrm{O}_{3}$ until the solution turned blue in color. The flask was purged with $\mathrm{O}_{2}$ until the blue color dissipated, and then $\mathrm{NaBH}_{4}(2.46 \mathrm{~g}, 65.1 \mathrm{mmol}, 3.0$ equiv) was added. The solution was allowed to slowly warm to rt overnight before it was slowly quenched with $\mathrm{H}_{2} \mathrm{O}$ (100 $\mathrm{mL})$. The slurry was extracted with $\mathrm{CH}_{2} \mathrm{Cl}_{2}(4 \times 50 \mathrm{~mL})$ and the combined organic phase was washed with brine, dried with $\mathrm{MgSO}_{4}$, filtered, and concentrated in vacuo. The resulting clear oil was subjected to the next step without further purification.

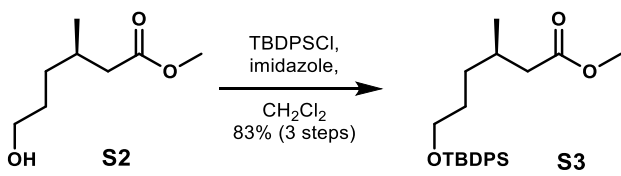

Methyl (R)-6-((tert-butyldiphenylsilyl)oxy)-3-methylhexanoate (S3):

To a solution of alcohol $\mathbf{S 2}$ (3.45 g, $21.7 \mathrm{mmol}, 1.0$ equiv) in $\mathrm{CH}_{2} \mathrm{Cl}_{2}(100 \mathrm{~mL})$ at rt was added imidazole (4.43 g, $65.1 \mathrm{mmol}, 3.0$ equiv) followed by $t$-butyl(chloro)diphenylsilane ( $8.5 \mathrm{~mL}, 32.6$ 
mmol, 1.5 equiv). The reaction was stirred at $\mathrm{rt}$ for $18 \mathrm{~h}$ and then diluted with $\mathrm{H}_{2} \mathrm{O}(100 \mathrm{~mL})$. The organic layer was extracted and additional extractions were performed with $\mathrm{CH}_{2} \mathrm{Cl}_{2}(2 \times 50 \mathrm{~mL})$. The combined organic phase was washed with brine, dried with $\mathrm{MgSO}_{4}$, filtered, and concentrated in vacuo. The resulting residue was purified via flash chromatography ( $2 \% \mathrm{EtOAc}$ in hex) to obtain ester $\mathbf{S} 3$ as a clear oil $(7.22 \mathrm{~g}, 83 \%$ yield over 3 steps).

${ }^{1} \mathrm{H}$ NMR $\left(500 \mathrm{MHz}, \mathrm{CDCl}_{3}\right): \delta 7.67(\mathrm{~d}, J=7.3 \mathrm{~Hz}, 4 \mathrm{H}), 7.46-7.36(\mathrm{~m}, 6 \mathrm{H}), 3.70-3.62(\mathrm{~m}$, $5 \mathrm{H}), 2.30(\mathrm{dd}, J=14.7,5.9 \mathrm{~Hz}, 1 \mathrm{H}), 2.12(\mathrm{dd}, J=14.7,8.2 \mathrm{~Hz}, 1 \mathrm{H}), 2.01-1.91(\mathrm{~m}, 1 \mathrm{H}), 1.65-$ $1.50(\mathrm{~m}, 2 \mathrm{H}$ ), 1.40 (ddd, $J=16.1,11.3,5.5 \mathrm{~Hz}, 1 \mathrm{H}$ ), 1.26 (ddd, $J=18.6,10.4,6.9 \mathrm{~Hz}, 1 \mathrm{H}$ ), 1.06 (s, 9H), $0.93(\mathrm{~d}, J=6.6 \mathrm{~Hz}, 3 \mathrm{H})$.

${ }^{13} \mathrm{C}\left\{{ }^{1} \mathrm{H}\right\}$ NMR $\left(126 \mathrm{MHz}, \mathrm{CDCl}_{3}\right): \delta 173.8,135.7,134.2,129.7,127.7,64.2,51.5,41.8,33.0$, $30.3,30.1,27.0,19.8,19.4$.

HRMS (ESI-TOF) m/z: Calcd for $\mathrm{C}_{24} \mathrm{H}_{35} \mathrm{O}_{3} \mathrm{Si}^{+}[\mathrm{M}+\mathrm{H}]^{+}$399.2355; Found 399.2353.

$$
[\alpha]^{22}{ }_{D}=+4.7\left(c=10.0, \mathrm{CHCl}_{3}\right) \text {. }
$$

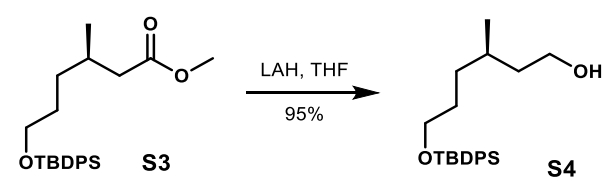

\section{(R)-6-((tert-Butyldiphenylsilyl)oxy)-3-methylhexan-1-ol (S4):}

To a solution of ester $\mathbf{S} 3(7.22 \mathrm{~g}, 18.8 \mathrm{mmol}, 1.0$ equiv) in THF at rt (60 mL) was added lithium aluminum hydride solution (1.0 M in THF; $22 \mathrm{~mL}, 22.6 \mathrm{mmol}, 1.2$ equiv). The reaction mixture was stirred for $15 \mathrm{~h}$ at $\mathrm{rt}$ and then slowly quenched with $\mathrm{H}_{2} \mathrm{O}(100 \mathrm{~mL})$. The slurry was extracted with $\mathrm{Et}_{2} \mathrm{O}(4 \times 100 \mathrm{~mL})$ and the combined organic phase was washed with brine, dried with $\mathrm{MgSO}_{4}$, filtered, and concentrated in vacuo to yield alcohol $\mathbf{S 4}$ as a clear oil $(6.40 \mathrm{~g}, 95 \%)$.

${ }^{1} \mathrm{H}$ NMR $\left(500 \mathrm{MHz}, \mathrm{CDCl}_{3}\right): \delta 7.68(\mathrm{~d}, J=7.8 \mathrm{~Hz}, 4 \mathrm{H}), 7.46-7.34(\mathrm{~m}, 6 \mathrm{H}), 3.72-3.60(\mathrm{~m}$, $4 \mathrm{H}), 1.67-1.50(\mathrm{~m}, 4 \mathrm{H}), 1.43-1.34(\mathrm{~m}, 2 \mathrm{H}), 1.25-1.20,(\mathrm{~m}, 1 \mathrm{H}), 1.06(\mathrm{~s}, 9 \mathrm{H}), 0.89(\mathrm{~d}, J=6.6$ $\mathrm{Hz}, 3 \mathrm{H})$.

${ }^{13} \mathrm{C}\left\{{ }^{1} \mathrm{H}\right\}$ NMR $\left(126 \mathrm{MHz}, \mathrm{CDCl}_{3}\right): \delta 135.7,134.3,129.7,127.7,64.3,61.3,40.0,33.2,30.1$, 29.4, 27.0, 19.8, 19.4 .

HRMS (ESI-TOF) m/z: Calcd for $\mathrm{C}_{23} \mathrm{H}_{34} \mathrm{O}_{2} \mathrm{SiNa}^{+}[\mathrm{M}+\mathrm{Na}]^{+}$393.2226; Found 393.2216.

$[\alpha]^{22}=+3.3\left(c=10.0, \mathrm{CHCl}_{3}\right)$. 


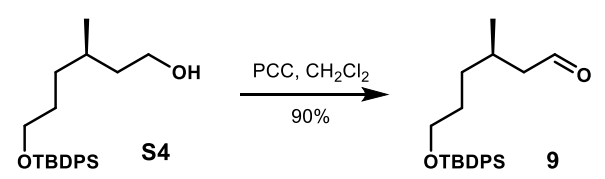

\section{(R)-6-((tert-Butyldiphenylsilyl)oxy)-3-methylhexanal (9):}

To a solution of alcohol S4 (6.40 g, $17.3 \mathrm{mmol}, 1.0$ equiv) in $\mathrm{CH}_{2} \mathrm{Cl}_{2}(80 \mathrm{~mL})$ at rt was added PCC $(6.70 \mathrm{~g}, 31.1 \mathrm{mmol}, 1.8$ equiv). The reaction mixture was stirred at $\mathrm{rt}$ for $2 \mathrm{~h}$, then it was filtered through a silica plug. The cake was washed with additional $\mathrm{CH}_{2} \mathrm{Cl}_{2}(300 \mathrm{~mL})$ and the filtrate was concentrated in vacuo to yield aldehyde 9 as a clear oil $(5.74 \mathrm{~g}, 90 \%)$.

${ }^{1} \mathrm{H}$ NMR $\left(500 \mathrm{MHz}, \mathrm{CDCl}_{3}\right): \delta 9.74(\mathrm{~s}, 1 \mathrm{H}), 7.68(\mathrm{~d}, J=6.8 \mathrm{~Hz}, 4 \mathrm{H}), 7.46-7.37(\mathrm{~m}, 6 \mathrm{H}), 3.67$ (t, $J=6.4 \mathrm{~Hz}, 2 \mathrm{H}$ ), 2.38 (dd, $J=16.1,5.5 \mathrm{~Hz}, 1 \mathrm{H}$ ), 2.22 (ddd, $J=16.1,8.0,2.4 \mathrm{~Hz}, 1 \mathrm{H}$ ), $2.10-$ $2.00(\mathrm{~m}, 1 \mathrm{H}), 1.66-1.50(\mathrm{~m}, 2 \mathrm{H}), 1.46-1.36(\mathrm{~m}, 1 \mathrm{H}), 1.36-1.25(\mathrm{~m}, 1 \mathrm{H}), 1.07(\mathrm{~s}, 9 \mathrm{H}), 0.96(\mathrm{~d}$, $J=6.7 \mathrm{~Hz}, 3 \mathrm{H})$.

${ }^{13} \mathrm{C}\left\{{ }^{1} \mathrm{H}\right\}$ NMR $\left(126 \mathrm{MHz}, \mathrm{CDCl}_{3}\right): \delta 203.1,135.7,134.1,129.7,127.8,64.0,51.1,33.1,30.0$, 28.0, 27.0, 20.1, 19.3 .

HRMS (ESI-TOF) m/z: Calcd for $\mathrm{C}_{23} \mathrm{H}_{32} \mathrm{O}_{2} \mathrm{SiNa}^{+}[\mathrm{M}+\mathrm{Na}]^{+}$391.2069; Found 391.2072.

$[\alpha]^{22}=+6.2\left(c=10.0, \mathrm{CHCl}_{3}\right)$.

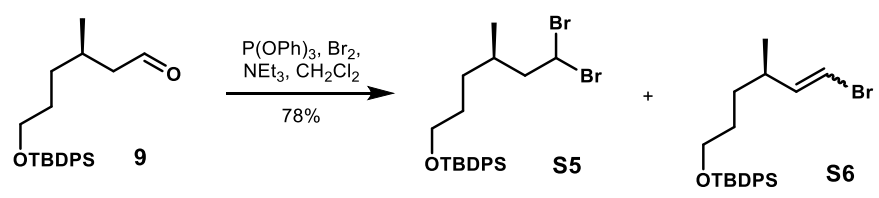

(R)-tert-Butyl((6,6-dibromo-4-methylhexyl)oxy)diphenylsilane (S5):

Dibromide S5 was made according to a modified literature procedure. ${ }^{2}$

To a solution of triphenyl phosphite $\left(6.1 \mathrm{~mL}, 23.4 \mathrm{mmol}, 1.5\right.$ equiv) in $\mathrm{CH}_{2} \mathrm{Cl}_{2}(100 \mathrm{~mL})$ at $78{ }^{\circ} \mathrm{C}$ was added $\mathrm{Br}_{2}\left(1.0 \mathrm{~mL}, 20.3 \mathrm{mmol}, 1.3\right.$ equiv) followed by $\mathrm{NEt}_{3}(6.5 \mathrm{~mL}, 46.8 \mathrm{mmol}, 3.0$ equiv) dropwise. When the foggy vapor disappeared from the head space, a solution of aldehyde 9 (5.74 g, $15.6 \mathrm{mmol}, 1.0$ equiv) in $\mathrm{CH}_{2} \mathrm{Cl}_{2}(50 \mathrm{~mL})$ was added via cannula transfer. The reaction was stirred for $2 \mathrm{~h}$ at $-78{ }^{\circ} \mathrm{C}$, then warmed to rt and stirred for an additional $2 \mathrm{~h}$. The reaction solution was concentrated in vacuo and subjected to flash column chromatography (1\% EtOAc in hex) to obtain dibromide $\mathbf{S 5}$ as an inconsequential and inseparable mixture of geminal dibromo alkane, and cis and trans vinyl bromide diastereomers S6 (10: $0.8: 1.3)$. (6.32 g, 79\%). This mixture is a clear oil. 
To remove excess $\mathrm{P}(\mathrm{OPh})_{3}$, for a simpler purification, the crude oil was stirred with $30 \%$ aq. $\mathrm{H}_{2} \mathrm{O}_{2}$ in THF for 20 min. The solution was diluted with brine and extracted with $\mathrm{Et}_{2} \mathrm{O}$. The combined organic phase was washed with brine, dried with $\mathrm{MgSO}_{4}$, filtered, and concentrated in vacuo. The resulting residue was purified via silica plug (10\% EtOAc in hex) to obtain geminal dibromide S5 as an inconsequential and inseparable mixture of the geminal dibromo alkane and cis and trans vinyl bromide diastereomers $\mathbf{S 6}(10: 0.8: 1.3)$.

${ }^{1} \mathrm{H}$ NMR $\left(500 \mathrm{MHz}, \mathrm{CDCl}_{3}\right): \delta 7.68(\mathrm{dd}, J=7.9,1.4 \mathrm{~Hz}, 4 \mathrm{H}), 7.46-7.36(\mathrm{~m}, 6 \mathrm{H}), 5.70(\mathrm{dd}, J$ $=8.4,5.9 \mathrm{~Hz}, 1 \mathrm{H}$ ), $3.67(\mathrm{t}, J=6.3 \mathrm{~Hz}, 2 \mathrm{H}$ ), 2.41 (ddd, $J=14.3,8.4,5.6 \mathrm{~Hz}, 1 \mathrm{H}$ ), 2.21 (ddd, $J=$ 15.0, 8.0, $5.5 \mathrm{~Hz}, 1 \mathrm{H}), 1.76(\mathrm{dd}, J=13.0,6.3 \mathrm{~Hz}, 1 \mathrm{H}), 1.64-1.51(\mathrm{~m}, 2 \mathrm{H}), 1.45-1.36(\mathrm{~m}, 1 \mathrm{H})$, $1.29-1.20(\mathrm{~m}, 1 \mathrm{H}), 1.07(\mathrm{~s}, 9 \mathrm{H}), 0.90(\mathrm{~d}, J=6.7 \mathrm{~Hz}, 3 \mathrm{H})$.

${ }^{13} \mathrm{C}\left\{{ }^{1} \mathrm{H}\right\}$ NMR $\left(126 \mathrm{MHz}, \mathrm{CDCl}_{3}\right) \delta 135.7,134.2,129.7,127.8,77.4,77.2,76.9,64.0,52.9$, 45.0, 32.4, 32.2, 29.7, 27.0, 19.4, 18.7 .

HRMS (Cl-TOF) m/z: Calcd for $\mathrm{C}_{23} \mathrm{H}_{32} \mathrm{Br}_{2} \mathrm{OSiH}^{+}[\mathrm{M}+\mathrm{H}]^{+}$513.0649; Found 513.0637.

\section{Diagnostic data for}

\section{(R)-tert-butyl((6,6-dibromo-4-methylhexyl)oxy)diphenylsilane (S6):}

${ }^{1} \mathrm{H}$ NMR $\left(500 \mathrm{MHz}, \mathrm{CDCl}_{3}\right) \delta 6.09(\mathrm{~d}, J=6.9 \mathrm{~Hz}, 1 \mathrm{H}, c i s), 6.04(\mathrm{dd}, J=13.5,8.1 \mathrm{~Hz}, 1 \mathrm{H}$, trans), $5.96(\mathrm{~d}, J=13.6 \mathrm{~Hz}, 1 \mathrm{H}$, trans), $5.85(\mathrm{dd}, J=9.2,7.0 \mathrm{~Hz}, 1 \mathrm{H}, c i s), 1.12(\mathrm{~d}, J=6.0 \mathrm{~Hz}, 3 \mathrm{H}$, cis), $1.00(\mathrm{~d}, J=6.7 \mathrm{~Hz}, 3 \mathrm{H}$, trans).

HRMS (Cl-TOF) m/z: Calcd for $\mathrm{C}_{19} \mathrm{H}_{22} \mathrm{BrOSi}^{+}\left[\mathrm{M}-{ }^{t} \text { butyl] }\right]^{+}$373.0623; Found 373.0634.

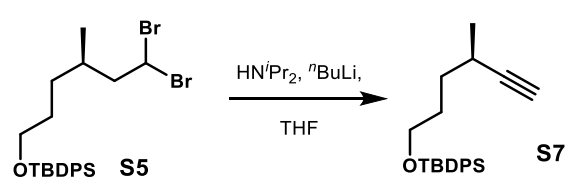

(R)-tert-butyl((4-methylhex-5-yn-1-yl)oxy)diphenylsilane (S7):

To a solution of $\mathrm{HN}^{\prime} \mathrm{Pr}_{2}\left(7.0 \mathrm{~mL}, 49.3 \mathrm{mmol}, 4.0\right.$ equiv) in THF $(20 \mathrm{~mL})$ at $-78{ }^{\circ} \mathrm{C}$ was added ${ }^{n} \mathrm{BuLi}\left(2.5 \mathrm{M}\right.$ in hex.; $17.0 \mathrm{~mL}, 43.2 \mathrm{mmol}, 3.5$ equiv). The solution was warmed to $0{ }^{\circ} \mathrm{C}$ then cooled back to $-78{ }^{\circ} \mathrm{C}$ after which dibromide $\mathbf{S} 5(6.32 \mathrm{~g}, 12.3 \mathrm{mmol}, 1.0$ equiv) in THF (20 mL) was added. The reaction mixture was stirred for $2 \mathrm{~h}$ at $-78{ }^{\circ} \mathrm{C}$, then warmed to it and stirred for an additional $3 \mathrm{~h}$. The reaction was quenched with saturated $\mathrm{NH}_{4} \mathrm{Cl}$ solution $(100 \mathrm{~mL})$. The mixture was extracted with $\mathrm{Et}_{2} \mathrm{O}(3 \times 40 \mathrm{~mL})$, and the combined organic phase was washed with brine, dried with $\mathrm{MgSO}_{4}$, filtered, and concentrated in vacuo. The light orange oil was taken on to the 
next step without further purification. For characterization, an aliquot of the resulting residue was purified through a silica plug (10\% EtOAc in hex) to obtain alkyne $\mathbf{S 7}$ as a clear oil. The spectroscopic data matches that of which has been previously reported. ${ }^{3}$

${ }^{1} \mathrm{H}$ NMR $\left(500 \mathrm{MHz}, \mathrm{CDCl}_{3}\right): \delta 7.68(\mathrm{~d}, J=6.5 \mathrm{~Hz}, 4 \mathrm{H}), 7.46-7.36(\mathrm{~m}, 6 \mathrm{H}), 3.69(\mathrm{t}, J=6.3$ $\mathrm{Hz}, 2 \mathrm{H}), 2.48-2.39(\mathrm{~m}, 1 \mathrm{H}), 2.03(\mathrm{~d}, J=2.3 \mathrm{~Hz}, 1 \mathrm{H}), 1.81-1.72(\mathrm{~m}, 1 \mathrm{H}), 1.72-1.63(\mathrm{~m}, 1 \mathrm{H})$, $1.61-1.49(\mathrm{~m}, 1 \mathrm{H}), 1.18(\mathrm{~d}, J=6.9 \mathrm{~Hz}, 3 \mathrm{H}), 1.06(\mathrm{~s}, 9 \mathrm{H})$.

${ }^{13} \mathrm{C}\left\{{ }^{1} \mathrm{H}\right\}$ NMR $\left(126 \mathrm{MHz}, \mathrm{CDCl}_{3}\right): \delta 135.7,134.2,129.7,127.8,89.2,68.4,63.8,33.2,30.4$, $27.0,25.6,21.1,19.4$.

HRMS (Cl-TOF) m/z: Calcd for $\mathrm{C}_{23} \mathrm{H}_{30} \mathrm{OSiH}^{+}[\mathrm{M}+\mathrm{H}]^{+}$351.2144; Found 351.2137.

$$
[\alpha]^{22}=-10.8\left(c=10.0, \mathrm{CHCl}_{3}\right) \text {. }
$$

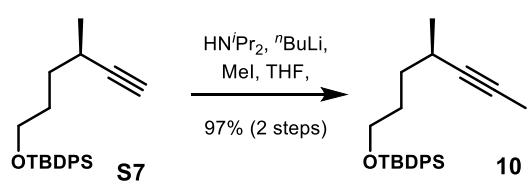

\section{(R)-tert-Butyl((4-methylhept-5-yn-1-yl)oxy)diphenylsilane (10):}

To a solution of $\mathrm{HN}^{\prime} \mathrm{Pr}_{2}(5.2 \mathrm{~mL}, 49.3 \mathrm{mmol}, 3.0$ equiv $)$ in THF $(20 \mathrm{~mL})$ at $-78{ }^{\circ} \mathrm{C}$ was added ${ }^{n} \mathrm{BuLi}$ (2.5 M in hex.; $12.0 \mathrm{~mL}, 30.8 \mathrm{mmol}, 2.5$ equiv). The solution was warmed to $0{ }^{\circ} \mathrm{C}$ then cooled back to $-78{ }^{\circ} \mathrm{C}$ after which alkyne $\mathbf{S 7}(4.31 \mathrm{~g}, 12.3 \mathrm{mmol}, 1.0$ equiv) in THF (40 mL) was added. The solution was warmed to $\mathrm{rt}$ and stirred for $1 \mathrm{~h}$ before it was cooled to $-78{ }^{\circ} \mathrm{C}$ and iodomethane $(5.3 \mathrm{~mL}, 86.1 \mathrm{mmol}, 7.0$ equiv) was added. The reaction was allowed to warm to $\mathrm{rt}$ over $14 \mathrm{~h}$ and then quenched with saturated $\mathrm{NH}_{4} \mathrm{Cl}$ solution $(100 \mathrm{~mL})$. The mixture was extracted with $\mathrm{Et}_{2} \mathrm{O}(3 \times 40 \mathrm{~mL})$, and the combined organic phase was washed with brine, dried with $\mathrm{MgSO}_{4}$, filtered, and concentrated in vacuo. The resulting residue was purified through a plug of silica gel (10\% EtOAc in hex) to obtain alkyne 10 (4.36 g, 97\% over 2 steps) as a clear oil.

${ }^{1} \mathrm{H}$ NMR $\left(500 \mathrm{MHz}, \mathrm{CDCl}_{3}\right): \delta 7.70(\mathrm{~d}, J=6.9 \mathrm{~Hz}, 4 \mathrm{H}), 7.47-7.36(\mathrm{~m}, 6 \mathrm{H}), 3.71(\mathrm{t}, J=6.4$ $\mathrm{Hz}, 2 \mathrm{H}), 2.43-2.34(\mathrm{~m}, 1 \mathrm{H}), 1.80(\mathrm{~d}, J=2.2 \mathrm{~Hz}, 3 \mathrm{H}), 1.78-1.72(\mathrm{~m}, 1 \mathrm{H}), 1.71-1.62(\mathrm{~m}, 1 \mathrm{H})$, 1.52 (ddd, $J=15.4,11.9,5.6 \mathrm{~Hz}, 1 \mathrm{H}), 1.46$ (ddd, $J=13.1,8.8,5.1 \mathrm{~Hz}, 1 \mathrm{H}), 1.15(\mathrm{~d}, J=6.9 \mathrm{~Hz}$, $3 \mathrm{H}), 1.08(\mathrm{~s}, 9 \mathrm{H})$.

${ }^{13} \mathrm{C}\left\{{ }^{1} \mathrm{H}\right\}$ NMR $\left(126 \mathrm{MHz}, \mathrm{CDCl}_{3}\right): \delta 135.7,134.3,129.6,127.7,83.9,75.8,64.0,33.7,30.6$, $27.0,25.8,21.6,19.4,3.64$.

HRMS (Cl-TOF) m/z: Calcd for $\mathrm{C}_{24} \mathrm{H}_{32} \mathrm{OSiH}^{+}[\mathrm{M}+\mathrm{H}]^{+}$365.2301; Found 365.2305. 
$[\alpha]^{22}=-9.1\left(c=10.0, \mathrm{CHCl}_{3}\right)$.

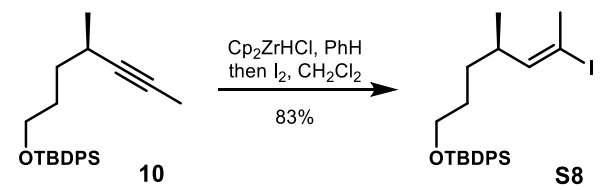

\section{(R,E)-tert-butyl((6-iodo-4-methylhept-5-en-1-yl)oxy)diphenylsilane (S8):}

Schwartz reagent $\left(\mathrm{Cp}_{2} \mathrm{ZrHCl}\right)$ was synthesized according to a known procedure and stored in a nitrogen filled glovebox. ${ }^{4}$

A Schlenk flask containing $\mathrm{Cp}_{2} \mathrm{ZrHCl}(6.16 \mathrm{~g}, 24.0 \mathrm{mmol}, 2.0$ equiv) was removed from the glove box, and a solution of alkyne 10 (4.36 g, $12.0 \mathrm{mmol}, 1.0$ equiv) in benzene (120 mL) was added at rt. After stirring for $17 \mathrm{~h}$ at $\mathrm{rt}$, a saturated solution of $\mathrm{I}_{2}$ in $\mathrm{CH}_{2} \mathrm{Cl}_{2}$ was added to the cloudy yellow mixture until the purple color persisted in the solution. A saturated $\mathrm{Na}_{2} \mathrm{~S}_{2} \mathrm{O}_{3}$ solution was added and the mixture was vigorously stirred until purple color dissipated. The slurry was diluted with brine and extracted with $\mathrm{CH}_{2} \mathrm{Cl}_{2}(4 \times 200 \mathrm{~mL})$. The combined organic phase was dried with $\mathrm{Na}_{2} \mathrm{SO}_{4}$, filtered, and concentrated in vacuo. The resulting residue was purified via flash chromatography $(0 \% \rightarrow 15 \%$ EtOAc in hex) to obtain vinyl iodide $\mathbf{S 8}$ as a yellow-orange oil (4.92 g, $83 \%)$.

${ }^{1} \mathrm{H}$ NMR $\left(500 \mathrm{MHz}, \mathrm{CDCl}_{3}\right): \delta 7.66(\mathrm{~d}, J=6.6 \mathrm{~Hz}, 4 \mathrm{H}), 7.45-7.36(\mathrm{~m}, 6 \mathrm{H}), 5.92$ (dd, $J=9.9$, $1.2 \mathrm{~Hz}, 1 \mathrm{H}), 3.63(\mathrm{t}, J=6.4 \mathrm{~Hz}, 2 \mathrm{H}), 2.39-2.33(\mathrm{~m}, 1 \mathrm{H}), 2.32(\mathrm{~d}, J=1.3 \mathrm{~Hz}, 3 \mathrm{H}), 1.58-1.44(\mathrm{~m}$, 2H), 1.38 (dd, $J=15.8,9.3,5.5 \mathrm{~Hz}, 1 \mathrm{H}), 1.27$ (dd, $J=18.7,9.1,4.4 \mathrm{~Hz}, 1 \mathrm{H}), 1.05$ (s, $9 \mathrm{H}), 0.94$ $(\mathrm{d}, J=6.7 \mathrm{~Hz}, 3 \mathrm{H})$.

${ }^{13} \mathrm{C}\left\{{ }^{1} \mathrm{H}\right\}$ NMR $\left(126 \mathrm{MHz}, \mathrm{CDCl}_{3}\right): \delta 147.5,135.7,134.2,129.7,127.8,92.8,63.9,35.6,33.2$, 30.5, 27.9, 27.0, 20.6, 19.4.

HRMS (Cl-TOF) m/z: Calcd for $\mathrm{C}_{24} \mathrm{H}_{33} \mathrm{OISiH}^{+}[\mathrm{M}+\mathrm{H}]$ 493.1424; Found 493.1447.

$$
[\alpha]^{22} \mathrm{D}=-24.8\left(c=1.0, \mathrm{CHCl}_{3}\right) .
$$
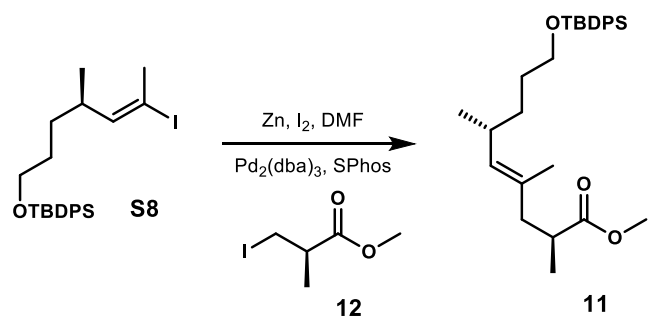

12 


\section{Methyl (2S,6R,E)-9-((tert-butyldiphenylsilyl)oxy)-2,4,6-trimethylnon-4-enoate (11):}

Ester 12 was prepared according to a known literature procedure. ${ }^{5}$

A Schlenk flask containing $\mathrm{Zn}$ dust (<10 micron; $2.60 \mathrm{~g}, 40.0 \mathrm{mmol}, 4.0$ equiv) was heated with a heat gun under vacuum for $10 \mathrm{~min}$. The flask was purged with $\mathrm{Ar}$ and was allowed to cool to rt. To the flask containing the $\mathrm{Zn}$ dust, DMF (Sparged with Ar for $1 \mathrm{~h}, 10 \mathrm{~mL}$ ) and $\mathrm{I}_{2}(380 \mathrm{mg}$ ) was added at rt and was stirred until the purple color dissipated. To this slurry, ester $12(2.96 \mathrm{~g}$, $13.0 \mathrm{mmol}, 1.3$ equiv) in DMF (15 mL) was added, followed by an additional portion of $\mathrm{I}_{2}$ (380 $\mathrm{mg})$. The slurry was stirred for $30 \mathrm{~min}$, after which time the purple color dissipated, then $\operatorname{Pd}_{2}(\mathrm{dba})_{3}$ (916 mg, $1.0 \mathrm{mmol}, 0.10$ equiv) and SPhos ( $821 \mathrm{mg}, 2.0 \mathrm{mmol}, 0.20$ equiv) were added followed by vinyl iodide $\mathbf{S} 8$ (4.92 g, $10.0 \mathrm{mmol}, 1.0$ equiv) in DMF ( $35 \mathrm{~mL})$. The reaction mixture was heated to $57^{\circ} \mathrm{C}$ in an oil bath for $14 \mathrm{~h}$. After cooling to rt, the slurry was diluted with EtOAc $(100 \mathrm{~mL})$ and then filtered through a pad of Celite and the cake washed with additional EtOAc (100 mL). The filtrate was washed with brine $(4 \times 100 \mathrm{~mL})$ and the combined organic phase was dried with $\mathrm{Na}_{2} \mathrm{SO}_{4}$, filtered, and concentrated in vacuo. The resulting residue was purified via flash chromatography (5\% EtOAc in hex) to obtain ester 11 (4.28 g, ca. $9.17 \mathrm{mmol}, 92 \%$ ) as a lightyellow oil, which was isolated with an unknown impurity and subjected to the next step without further purification.

${ }^{1} \mathrm{H}$ NMR $\left(500 \mathrm{MHz}, \mathrm{CDCl}_{3}\right): \delta 7.70(\mathrm{~d}, J=7.9 \mathrm{~Hz}, 4 \mathrm{H}), 7.47-7.38(\mathrm{~m}, 6 \mathrm{H}), 4.95(\mathrm{~d}, J=9.4$ $\mathrm{Hz}, 1 \mathrm{H}), 3.69-3.65(\mathrm{~m}, 5 \mathrm{H}), 2.70-2.60(\mathrm{~m}, 1 \mathrm{H}), 2.41-2.28(\mathrm{~m}, 2 \mathrm{H}), 2.05(\mathrm{dd}, J=13.5,7.8 \mathrm{~Hz}$, $1 \mathrm{H}), 1.59(\mathrm{~s}, 3 \mathrm{H}), 1.57-1.47(\mathrm{~m}, 2 \mathrm{H}), 1.44-1.37(\mathrm{~m}, 1 \mathrm{H}), 1.28-1.24(\mathrm{~m}, 1 \mathrm{H}), 1.13(\mathrm{~d}, J=7.0$ $\mathrm{Hz}, 3 \mathrm{H}), 1.08(\mathrm{~s}, 9 \mathrm{H}), 0.92(\mathrm{~d}, J=6.7 \mathrm{~Hz}, 3 \mathrm{H})$.

${ }^{13} \mathrm{C}\left\{{ }^{1} \mathrm{H}\right\}$ NMR $\left(126 \mathrm{MHz}, \mathrm{CDCl}_{3}\right): \delta 177.1,135.7,134.3,134.1,130.7,129.6,127.7,77.4,77.2$, $76.9,64.2,51.5,44.3,38.1,33.8,32.3,30.7,27.0,21.4,19.3,16.7,16.0$.

HRMS (ESI-TOF) m/z: Calcd for $\mathrm{C}_{29} \mathrm{H}_{42} \mathrm{O}_{3} \mathrm{SiH}^{+}[\mathrm{M}+\mathrm{H}]^{+}$467.2982; Found 467.2978.

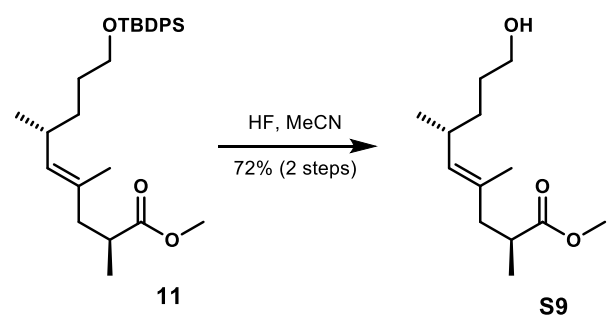

Methyl (2S,6R,E)-9-hydroxy-2,4,6-trimethylnon-4-enoate (S9): 
To a plastic bottle containing ester 11 (4.28 g, ca. $9.17 \mathrm{mmol}, 1.0$ equiv) in $\mathrm{MeCN}$ (37 mL) was added HF (50\% aq. soln; $1.0 \mathrm{~mL})$. After stirring for $1 \mathrm{~h}$, an additional portion of HF (2 mL) was added. After stirring for $3 \mathrm{~h}$, an additional portion of $\mathrm{HF}(5 \mathrm{~mL})$ was added. After stirring for $4.5 \mathrm{~h}$, an additional portion of HF (2 mL) was added. After stirring for $5 \mathrm{~h}$ total, the reaction was slowly quenched with saturated $\mathrm{NaHCO}_{3}$ solution and the mixture was extracted with ether $(3 \mathrm{x}$ $50 \mathrm{~mL}$ ). The combined organic phase was washed with brine, dried with $\mathrm{MgSO}_{4}$, filtered, and concentrated in vacuo. The resulting residue was purified through a silica plug $(30 \% \mathrm{EtOAc}$ in hex then $100 \%$ EtOAc) to obtain alcohol $\mathbf{S 9}(1.51 \mathrm{~g}, 72 \%$ over 2 steps) as a clear oil.

${ }^{1} \mathrm{H}$ NMR $\left(500 \mathrm{MHz}, \mathrm{CDCl}_{3}\right): \delta 4.83(\mathrm{~d}, J=9.5 \mathrm{~Hz}, 1 \mathrm{H}), 3.56(\mathrm{~s}, 3 \mathrm{H}), 3.50(\mathrm{t}, J=6.7 \mathrm{~Hz}, 2 \mathrm{H})$, $2.60-2.50$ (m, 1H), 2.34 (s, br, 1H), $2.28-2.19(\mathrm{~m}, 2 \mathrm{H}), 1.95$ (dd, J = 13.6, $7.4 \mathrm{~Hz}, 1 \mathrm{H}), 1.51$ (s, $3 \mathrm{H}), 1.47-1.36(\mathrm{~m}, 2 \mathrm{H}), 1.28$ (ddd, $J=16.0,9.2,5.5 \mathrm{~Hz}, 1 \mathrm{H}), 1.13$ (ddd, $J=13.4,8.7,5.1 \mathrm{~Hz}$, $1 \mathrm{H}), 1.03(\mathrm{~d}, J=7.1,3 \mathrm{H}), 0.83(\mathrm{~d}, J=6.9,3 \mathrm{H})$.

${ }^{13} \mathrm{C}\left\{{ }^{1} \mathrm{H}\right\}$ NMR $\left(126 \mathrm{MHz}, \mathrm{CDCl}_{3}\right): \delta 177.1,133.7,130.8,62.9,51.4,44.1,38.0,33.7,32.2$, $30.8,21.2,16.6,15.9$.

HRMS (ESI-TOF) $\mathrm{m} / \mathrm{z}$ : Calcd for $\mathrm{C}_{13} \mathrm{H}_{24} \mathrm{O}_{3} \mathrm{Na}^{+}[\mathrm{M}+\mathrm{Na}]^{+}$251.1623; Found 251.1624.

$[\alpha]_{D}^{22}=-6.3\left(c=10.0, \mathrm{CHCl}_{3}\right)$.

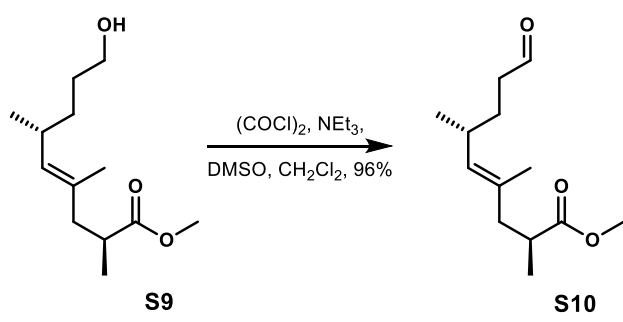

\section{Methyl (2S,6R,E)-2,4,6-trimethyl-9-oxonon-4-enoate (S10):}

To a solution of oxalyl chloride $\left(0.14 \mathrm{~mL}, 1.58 \mathrm{mmol}, 1.2\right.$ equiv) in $\mathrm{CH}_{2} \mathrm{Cl}_{2}(3.6 \mathrm{~mL})$ at $-78{ }^{\circ} \mathrm{C}$ was added DMSO $\left(0.22 \mathrm{~mL}, 3.14 \mathrm{mmol}, 2.4\right.$ equiv). The mixture was stirred at $-78{ }^{\circ} \mathrm{C}$ for $5 \mathrm{~min}$ after which alcohol $\mathbf{S 9}$ (300 mg, $1.31 \mathrm{mmol}, 1.0$ equiv) was added in $\mathrm{CH}_{2} \mathrm{Cl}_{2}$ (3.0 mL) followed by $\mathrm{NEt}_{3}(0.91 \mathrm{~mL}, 6.55 \mathrm{mmol}, 5.0$ equiv). The solution was warmed to rt, stirred for $20 \mathrm{~min}$, then quenched with saturated $\mathrm{NH}_{4} \mathrm{Cl}$ solution. The slurry was extracted with $\mathrm{CH}_{2} \mathrm{Cl}_{2}(3 \times 40 \mathrm{~mL})$, and the combined organic phase was washed with brine, dried with $\mathrm{Na}_{2} \mathrm{SO}_{4}$, filtered, and concentrated in vacuo to obtain aldehyde $\mathbf{S} 10$ (285 $\mathrm{mg}, 96 \%)$ as a light yellow oil.

${ }^{1} \mathrm{H}$ NMR $\left(500 \mathrm{MHz}, \mathrm{CDCl}_{3}\right): \delta 9.72(\mathrm{~s}, 1 \mathrm{H}), 4.86(\mathrm{~d}, J=9.6 \mathrm{~Hz}, 1 \mathrm{H}), 3.63(\mathrm{~s}, 3 \mathrm{H}), 2.66-2.57$ 
(m, 1H), $2.40-2.28(\mathrm{~m}, 4 \mathrm{H}), 2.02$, (dd, $J=13.6,7.3 \mathrm{~Hz}, 1 \mathrm{H}), 1.71-1.62(\mathrm{~m}, 1 \mathrm{H}), 1.56(\mathrm{~s}, 3 \mathrm{H})$, $1.50-1.40(\mathrm{~m}, 1 \mathrm{H}), 1.10(\mathrm{~d}, J=7.0 \mathrm{~Hz}, 1 \mathrm{H}), 0.92(\mathrm{~d}, J=6.7 \mathrm{~Hz}, 1 \mathrm{H})$.

${ }^{13} \mathrm{C}\left\{{ }^{1} \mathrm{H}\right\}$ NMR $\left(126 \mathrm{MHz}, \mathrm{CDCl}_{3}\right): \delta 202.8,177.0,132.5,132.3,51.5,44.2,42.3,38.1,32.1$, $29.7,21.3,16.9,16.2$.

HRMS (ESI-TOF) m/z: Calcd for $\mathrm{C}_{13} \mathrm{H}_{22} \mathrm{O}_{3} \mathrm{Na}^{+}[\mathrm{M}+\mathrm{Na}]^{+}$249.1467; Found 249.1477.

$[\alpha]^{22}=-4.5\left(c=10.0, \mathrm{CHCl}_{3}\right)$.

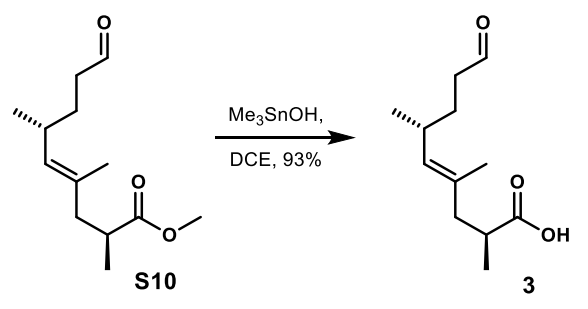

$(2 S, 6 R, E)-2,4,6$-trimethyl-9-oxonon-4-enoic acid (3):

Carboxylic acid 3 was prepared according to a modified literature procedure. ${ }^{6}$

To a solution of aldehyde $\mathbf{S} 10$ (270 mg, $1.19 \mathrm{mmol}, 1.0$ equiv) in dichloroethane $(6.0 \mathrm{~mL})$ was added trimethyltin hydroxide $\left(2.0 \mathrm{~g}, 11.1 \mathrm{mmol}, 9.3\right.$ equiv) and the slurry was heated to $85^{\circ} \mathrm{C}$ in an oil bath for $18.5 \mathrm{~h}$. The solution was cooled to rt, diluted with $\mathrm{CH}_{2} \mathrm{Cl}_{2}(10 \mathrm{~mL})$, and washed with $3 \mathrm{M} \mathrm{HCl}$ solution $(3 \times 10 \mathrm{~mL})$. The organic phase was dried with $\mathrm{Na}_{2} \mathrm{SO}_{4}$, filtered, and concentrated in vacuo to obtain carboxylic acid 3 (236 mg, 93\%) as a gummy, clear oil which was taken on to the next step without further purification.

${ }^{1} \mathrm{H}$ NMR $\left(500 \mathrm{MHz}, \mathrm{CDCl}_{3}\right): \delta 9.73(\mathrm{t}, J=1.6 \mathrm{~Hz}, 1 \mathrm{H}), 4.91(\mathrm{~d}, J=9.6 \mathrm{~Hz}, 1 \mathrm{H}), 2.64-2.60$ $(\mathrm{m}, 1 \mathrm{H}), 2.40-2.32(\mathrm{~m}, 4 \mathrm{H}), 2.06(\mathrm{dd}, J=13.6,7.6 \mathrm{~Hz}, 1 \mathrm{H}), 1.68(\mathrm{ddd}, J=10.0,7.7,4.1 \mathrm{~Hz}, 1 \mathrm{H})$, 1.59 (s, 3H), 1.48 (ddd, $J=11.2,8.2,6.1 \mathrm{~Hz}, 1 \mathrm{H}), 1.13(\mathrm{~d}, J=6.9 \mathrm{~Hz}, 3 \mathrm{H}), 0.93(\mathrm{~d}, J=6.7 \mathrm{~Hz}$, $3 \mathrm{H})$.

${ }^{13} \mathrm{C}\left\{{ }^{1} \mathrm{H}\right\} \operatorname{NMR}\left(126 \mathrm{MHz}, \mathrm{CDCl}_{3}\right): \delta 203.0,182.9,132.8,132.0,43.7,42.2,38.0,32.1,29.7$, $21.2,16.5,16.1$.

HRMS (ESI-TOF) m/z: Calcd for $\mathrm{C}_{12} \mathrm{H}_{19} \mathrm{O}_{3}{ }^{-}[\mathrm{M}+\mathrm{Na}]^{-}$211.1334; Found 211.1335.

$[\alpha]^{22}=-14.0\left(c=10.0, \mathrm{CHCl}_{3}\right)$. 


\section{Synthesis of Vinyl lodide Coupling Partner:}

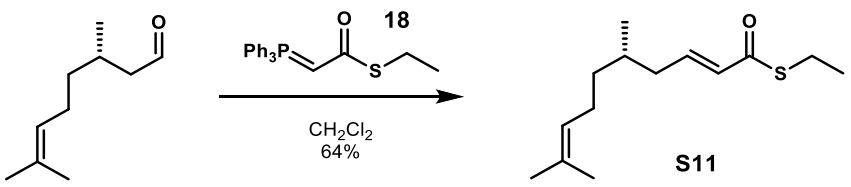

\section{(S)-Ethyl (S,E)-5,9-dimethyldeca-2,8-dienethioate (S11):}

Ylide 18 was prepared using known literature procedures. ${ }^{7}$

To a solution of ylide 18 (12.6 g, $34.7 \mathrm{mmol}, 1.0$ equiv) in $\mathrm{CH}_{2} \mathrm{Cl}_{2}(210 \mathrm{~mL})$ at rt was added (S)-citronellal (8) $\left(6.4 \mathrm{~mL}, 41.6 \mathrm{mmol}, 1.2\right.$ equiv). The solution was heated to $50{ }^{\circ} \mathrm{C}$ in an oil bath and refluxed for $24 \mathrm{~h}$. The reaction mixture was cooled to rt and concentrated in vacuo. The yellow, chalky residue was purified via flash chromatography $\left(40 \% \mathrm{CH}_{2} \mathrm{Cl}_{2}\right.$ in hex) to obtain the thioester S11 as a yellow oil $(6.45 \mathrm{~g}, 64 \%)$.

${ }^{1} \mathrm{H}$ NMR $\left(500 \mathrm{MHz}, \mathrm{CDCl}_{3}\right): \delta 6.83(\mathrm{dt}, J=15.3,7.5 \mathrm{~Hz}, 1 \mathrm{H}), 6.07(\mathrm{dt}, J=15.5,1.4 \mathrm{~Hz}, 1 \mathrm{H})$, 5.05 (tt, $J=7.0,1.5 \mathrm{~Hz}, 1 \mathrm{H}$ ), 2.91 (q, J = 7.4 Hz, 2H), 2.17 (dddd, $J=14.3,7.2,5.7,1.4 \mathrm{~Hz}, 1 \mathrm{H}$ ), $2.07-1.87(\mathrm{~m}, 3 \mathrm{H}), 1.65(\mathrm{~s}, 3 \mathrm{H}), 1.62(\mathrm{dd}, J=13.2,6.6 \mathrm{~Hz}, 1 \mathrm{H}), 1.58(\mathrm{~s}, 3 \mathrm{H}), 1.38-1.28(\mathrm{~m}$, $1 \mathrm{H}), 1.28-1.23(\mathrm{t}, J=7 / 0 \mathrm{~Hz}, 3 \mathrm{H}), 1.20-1.13(\mathrm{~m}, 1 \mathrm{H}), 0.88(\mathrm{~d}, J=6.9 \mathrm{~Hz}, 3 \mathrm{H})$.

${ }^{13} \mathrm{C}\left\{{ }^{1} \mathrm{H}\right\}$ NMR $\left(126 \mathrm{MHz}, \mathrm{CDCl}_{3}\right): \delta 189.9,144.1,131.5,129.9,124.4,39.7,36.8,32.3,25.8$, $25.6,23.1,19.6,17.7,14.9$.

HRMS (Cl-TOF) m/z: Calcd for $\mathrm{C}_{14} \mathrm{H}_{24} \mathrm{OS}^{+}[\mathrm{M}]^{+} 240.1548$; Found 240.1556.

$$
[\alpha]^{22}=+3.8\left(c=10.0, \mathrm{CHCl}_{3}\right) \text {. }
$$

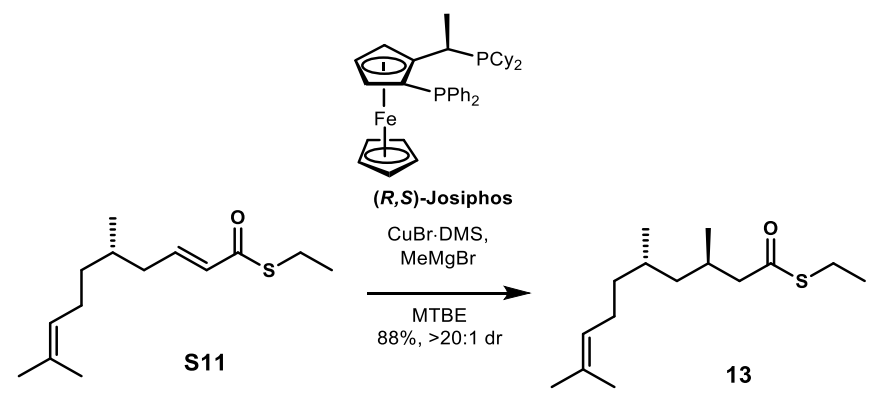

S-Ethyl (3R,5S)-3,5,9-trimethyldec-8-enethioate (13):

Thioester 13 was synthesized according to a modified literature procedure. ${ }^{8}$

A solution of CuBr-DMS (160 mg, $0.780 \mathrm{mmol}, 0.03$ equiv) and (R,S)-Josiphos (666 mg, 1.04 mmol. 0.04 equiv) in MTBE (240 mL) was stirred at it for 30 min. The mixture was cooled to - 
$78^{\circ} \mathrm{C}$ and $\mathrm{MeMgBr}^{9}$ (3.0 M solution in $\mathrm{Et}_{2} \mathrm{O} ; 15.5 \mathrm{~mL}, 46.6 \mathrm{mmol}, 1.8$ equiv) was added dropwise. After stirring for $15 \mathrm{~min}$, a solution of thioester $\mathbf{S 1 1}(6.23 \mathrm{~g}, 25.9 \mathrm{mmol}, 1.0$ equiv) in MTBE (20 $\mathrm{mL}$ ) was added via a syringe pump over $4 \mathrm{~h}$. The reaction mixture was stirred at $-78{ }^{\circ} \mathrm{C}$ for $16 \mathrm{~h}$. After full consumption of the starting material, indicated by TLC, the reaction was quenched by the addition of $\mathrm{MeOH}$ and allowed to warm to rt. $\mathrm{A}$ saturated $\mathrm{NH}_{4} \mathrm{Cl}$ solution was then added, the phases were separated, and the aqueous layer extracted with $\mathrm{Et}_{2} \mathrm{O}(3 \times 50 \mathrm{~mL})$. The combined organic phases were dried over $\mathrm{MgSO}_{4}$, concentrated in vacuo and purified by column flash chromatography ( $40 \% \mathrm{CH}_{2} \mathrm{Cl}_{2}$ in hex) to afford thioester 13 as a clear, colorless oil $(5.84 \mathrm{~g}, 88 \%$, $>20: 1 \mathrm{dr}$ ) containing $7 \mathrm{~mol} \%$ of unreacted cis-alkene starting material, formed through isomerization of the olefin under the reaction conditions.

${ }^{1} \mathrm{H}$ NMR $\left(600 \mathrm{MHz}, \mathrm{CDCl}_{3}\right) \delta 5.08$ (tt, $\left.J=7.2,1.2 \mathrm{~Hz}, 1 \mathrm{H}\right), 2.86$ (q, $J=7.4 \mathrm{~Hz}, 2 \mathrm{H}$ ), 2.47 (dd, $J=14.4,6.1 \mathrm{~Hz}, 1 \mathrm{H}), 2.34(\mathrm{dd}, J=14.4,8.0 \mathrm{~Hz}, 1 \mathrm{H}), 2.16-2.06(\mathrm{~m}, 1 \mathrm{H}), 2.02-1.87(\mathrm{~m}, 2 \mathrm{H})$, $1.67(\mathrm{~s}, 3 \mathrm{H}), 1.59(\mathrm{~s}, 3 \mathrm{H}), 1.51-1.43(\mathrm{~m}, 1 \mathrm{H}), 1.31-1.25(\mathrm{~m}, 1 \mathrm{H}), 1.24(\mathrm{t}, J=7.4 \mathrm{~Hz}, 3 \mathrm{H}), 1.18$ $-1.05(\mathrm{~m}, 3 \mathrm{H}), 0.89$ (d, $J=6.6 \mathrm{~Hz}, 3 \mathrm{H}), 0.85(\mathrm{~d}, J=6.6 \mathrm{~Hz}, 3 \mathrm{H})$.

${ }^{13} \mathrm{C}\left\{{ }^{1} \mathrm{H}\right\}$ NMR $\left(151 \mathrm{MHz}, \mathrm{CDCl}_{3}\right) \delta 199.3,131.2,125.0,52.3,44.2,37.8,29.8,28.7,25.8,25.6$, 23.4, 19.4, 19.4, 17.8, 15.0.

HRMS (Cl-TOF) m/z: Calcd for $\mathrm{C}_{15} \mathrm{H}_{28} \mathrm{OSH}^{+}[\mathrm{M}+\mathrm{H}]^{+}$257.1939; Found 257.1949.

$[\alpha]^{22}=+22.3\left(c=10.0, \mathrm{CHCl}_{3}\right)$.

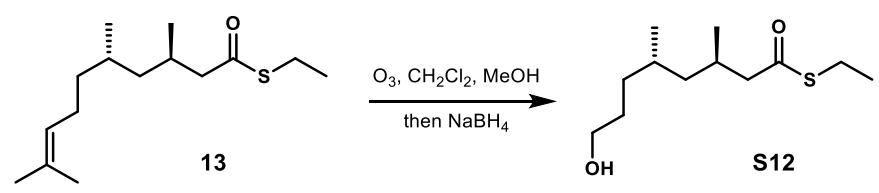

\section{S-Ethyl (3R,5S)-8-hydroxy-3,5-dimethyloctanethioate (S12):}

A solution of thioester 13 (3.64 g, $14.2 \mathrm{mmol}, 1.0$ equiv) in $\mathrm{CH}_{2} \mathrm{Cl}_{2}$ (53 mL) and $\mathrm{MeOH}(18 \mathrm{~mL})$ at $-78{ }^{\circ} \mathrm{C}$ was treated with $\mathrm{O}_{3}$ until the solution turned blue in color. The flask was purged with $\mathrm{O}_{2}$ until the blue color dissipated, and then $\mathrm{NaBH}_{4}(1.61 \mathrm{~g}, 42.6 \mathrm{mmol}, 3.0$ equiv) was added. The solution slowly warmed to rt overnight before it was slowly quenched with $\mathrm{H}_{2} \mathrm{O}(100 \mathrm{~mL})$ and stirred vigorously until bubbling ceased. The slurry was extracted with $\mathrm{CH}_{2} \mathrm{Cl}_{2}(4 \times 50 \mathrm{~mL})$ and the combined organic phase was washed with brine, dried with $\mathrm{Na}_{2} \mathrm{SO}_{4}$, filtered, and concentrated in vacuo. The resulting cloudy oil was subjected to the next step without further purification. An aliquot was reserved for purification by flash chromatography (50\% EtOAc in hex) to obtain alcohol S12. 
${ }^{1} \mathrm{H}$ NMR $\left(500 \mathrm{MHz}, \mathrm{CDCl}_{3}\right) \delta 3.50(\mathrm{t}, J=6.7 \mathrm{~Hz}, 2 \mathrm{H}), 2.78(\mathrm{q}, J=7.4 \mathrm{~Hz}, 2 \mathrm{H}), 2.52(\mathrm{~s}, 1 \mathrm{H})$, 2.39 (dd, $J=14.4,6.2 \mathrm{~Hz}, 1 \mathrm{H}$ ), $2.27(\mathrm{dd}, J=14.5,7.8 \mathrm{~Hz}, 1 \mathrm{H}), 2.02(\mathrm{td}, J=14.3,6.6 \mathrm{~Hz}, 1 \mathrm{H}$ ), $1.57-1.33(\mathrm{~m}, 3 \mathrm{H}), 1.23$ (ddt, $J=13.2,10.9,5.5 \mathrm{~Hz}, 1 \mathrm{H}), 1.15$ (t, $J=7.5 \mathrm{~Hz}, 3 \mathrm{H}), 1.12-1.00$ (m, 3H), $0.82(\mathrm{~d}, J=6.7 \mathrm{~Hz}, 3 \mathrm{H}), 0.78(\mathrm{~d}, J=6.7 \mathrm{~Hz}, 3 \mathrm{H})$.

${ }^{13} \mathrm{C}\left\{{ }^{1} \mathrm{H}\right\}$ NMR $\left(126 \mathrm{MHz}, \mathrm{CDCl}_{3}\right) \delta 199.5,63.3,52.2,44.3,33.6,30.3,30.0,28.7,23.4,19.4$, 19.4, 14.9.

HRMS (Cl-TOF) m/z: Calcd for $\mathrm{C}_{12} \mathrm{H}_{24} \mathrm{O}_{2} \mathrm{SH}^{+}[\mathrm{M}+\mathrm{H}]^{+}$233.1575; Found 233.1577.

$$
[\alpha]^{22}=+13.9\left(c=1.0, \mathrm{CHCl}_{3}\right) \text {. }
$$

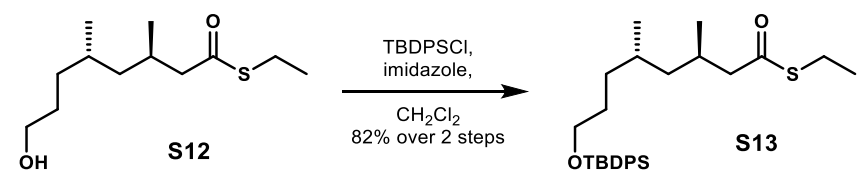

\section{S-Ethyl (3R,5S)-8-((tert-butyldiphenylsilyl)oxy)-3,5-dimethyloctanethioate (S13):}

To a flask containing $\mathbf{S} 12\left(2.00 \mathrm{~g}, 8.61 \mathrm{mmol}, 1.0\right.$ equiv) in $\mathrm{CH}_{2} \mathrm{Cl}_{2}(43 \mathrm{~mL})$ was added imidazole ( $878 \mathrm{mg}, 12.9 \mathrm{mmol}, 1.5$ equiv) followed by $t$-butyl(chloro)diphenylsilane (3.36 mL, 12.9 mmol, 1.5 equiv) dropwise at rt. The reaction mixture was stirred for $18 \mathrm{~h}$ at $\mathrm{rt}$ and then quenched with a saturated $\mathrm{NH}_{4} \mathrm{Cl}$ solution $(15 \mathrm{~mL}$ ). The organic layer was extracted, and additional extractions were performed with $\mathrm{CH}_{2} \mathrm{Cl}_{2}(2 \times 50 \mathrm{~mL})$. The organic phases were combined, washed with brine, dried with $\mathrm{MgSO}_{4}$, filtered, and concentrated in vacuo. The crude oil was purified via flash chromatography $\left(0 \rightarrow 40 \% \mathrm{CH}_{2} \mathrm{Cl}_{2}\right.$ in hex) to obtain thioester $\mathbf{S} 13$ as a clear, colorless oil (3.34 $\mathrm{g}, 82 \%$ yield over two steps).

${ }^{1} \mathrm{H}$ NMR $\left(600 \mathrm{MHz}, \mathrm{CDCl}_{3}\right) \delta 7.70(\mathrm{~d}, J=6.6 \mathrm{~Hz}, 4 \mathrm{H}), 7.46-7.37(\mathrm{~m}, 6 \mathrm{H}), 3.67(\mathrm{t}, J=6.6 \mathrm{~Hz}$, 2H), 2.89 (q, J = 7.4 Hz, 2H), 2.49 (dd, $J=14.5,6.2 \mathrm{~Hz}, 1 \mathrm{H}), 2.38(\mathrm{dd}, J=14.4,7.9 \mathrm{~Hz}, 1 \mathrm{H}), 2.13$ (td, $J=14.3,6.6 \mathrm{~Hz}, 1 \mathrm{H}), 1.63-1.45(\mathrm{~m}, 3 \mathrm{H}), 1.37-1.30(\mathrm{~m}, 1 \mathrm{H}), 1.27(\mathrm{t}, J=7.4 \mathrm{~Hz}, 3 \mathrm{H}), 1.24$ $-1.17(\mathrm{~m}, 1 \mathrm{H}), 1.13$ (ddd, $J=10.4,9.1,5.0 \mathrm{~Hz}, 2 \mathrm{H}), 1.08(\mathrm{~s}, 9 \mathrm{H}), 0.91(\mathrm{~d}, J=6.6 \mathrm{~Hz}, 3 \mathrm{H}), 0.86$ (d, $J=6.6 \mathrm{~Hz}, 3 \mathrm{H})$.

${ }^{13} \mathrm{C}\left\{{ }^{1} \mathrm{H}\right\}$ NMR $\left(151 \mathrm{MHz}, \mathrm{CDCl}_{3}\right) \delta 199.3,135.7,134.3,129.6,127.7,64.4,52.3,44.2,33.8$, $30.1,29.9,28.7,27.0,23.4,19.4(2 X), 19.3,15.0$.

HRMS (ESI-TOF) m/z: Calcd for $\mathrm{C}_{28} \mathrm{H}_{42} \mathrm{O}_{2} \mathrm{SSiNa}^{+}[\mathrm{M}+\mathrm{Na}]^{+}$493.2573; Found 493.2565.

$$
[\alpha]^{22}{ }_{D}=+8.9\left(c=10.0, \mathrm{CHCl}_{3}\right) \text {. }
$$




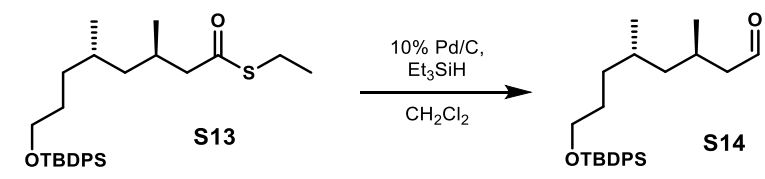

(3R,5S)-8-((tert-Butyldiphenylsilyl)oxy)-3,5-dimethyloctanal (S14):

To a solution of thioester $\mathbf{S} 13$ (942 mg, $2.00 \mathrm{~mol}, 1.0$ equiv) and $\mathrm{Et}_{3} \mathrm{SiH}$ (0.96 mL, $6 \mathrm{mmol}, 2.0$ equiv) in $\mathrm{CH}_{2} \mathrm{Cl}_{2}(4 \mathrm{~mL})$ at $\mathrm{rt}$ open to air was added 10\% $\mathrm{Pd} / \mathrm{C}$ (106 mg divided, $0.1 \mathrm{mmol}, 5 \mathrm{~mol} \%$ ) in three portions, each added after bubbling ceased. After full consumption of the starting material, indicated by TLC, (15-30 $\mathrm{min}$; up to $5 \mathrm{~mol} \%$ catalyst), the slurry was filtered over Celite and the cake was rinsed thoroughly with $\mathrm{CH}_{2} \mathrm{Cl}_{2}$. The filtrate was concentrated in vacuo to obtain aldehyde S14. The resulting yellow oil was carried forward without further purification. An analytical aliquot was reserved for purification by flash chromatography $\left(0 \rightarrow 40 \% \mathrm{CH}_{2} \mathrm{Cl}_{2}\right.$ in hex).

${ }^{1} \mathrm{H}$ NMR $\left(600 \mathrm{MHz}, \mathrm{CDCl}_{3}\right) \delta 9.74(\mathrm{t}, \mathrm{J}=2.3 \mathrm{~Hz}, 1 \mathrm{H}), 7.70-7.65(\mathrm{~m}, 4 \mathrm{H}), 7.39(\mathrm{dt}, \mathrm{J}=14.2$, $7.1 \mathrm{~Hz}, 6 \mathrm{H}$ ), $3.64(\mathrm{t}, J=6.5 \mathrm{~Hz}, 2 \mathrm{H}), 2.34$ (ddd, $J=16.0,5.8,2.1 \mathrm{~Hz}, 1 \mathrm{H}), 2.23$ (ddd, $J=16.0$, 7.7, $2.6 \mathrm{~Hz}, 1 \mathrm{H}), 2.14(\mathrm{td}, J=14.0,6.5 \mathrm{~Hz}, 1 \mathrm{H}), 1.63-1.43(\mathrm{~m}, 5 \mathrm{H}), 1.31$ (dtd, $J=15.9,10.7,5.4$ $\mathrm{Hz}, 1 \mathrm{H}$ ), 1.17 (tdd, $J=13.3,8.3,4.7 \mathrm{~Hz}, 2 \mathrm{H}), 1.08$ (dd, $J=9.0,5.0 \mathrm{~Hz}, 1 \mathrm{H}), 1.05(\mathrm{~s}, 9 \mathrm{H}), 0.91$ (d, $J=6.6 \mathrm{~Hz}, 3 \mathrm{H}), 0.85(\mathrm{~d}, J=6.6 \mathrm{~Hz}, 3 \mathrm{H})$.

${ }^{13} \mathrm{C}\left\{{ }^{1} \mathrm{H}\right\}$ NMR $\left(151 \mathrm{MHz}, \mathrm{CDCl}_{3}\right) \delta 203.2,135.7,134.3,129.7,127.7,64.3,51.9,44.60,33.7$, $30.1,29.9,27.02,25.8,19.8,19.4,19.4$.

HRMS (ESI-TOF) m/z: Calcd for $\mathrm{C}_{26} \mathrm{H}_{38} \mathrm{O}_{2} \mathrm{SiNa}^{+}[\mathrm{M}+\mathrm{Na}]^{+}$433.2593; Found 433.2559.

$[\alpha]^{22}=+15.6\left(c=10.0, \mathrm{CHCl}_{3}\right)$.

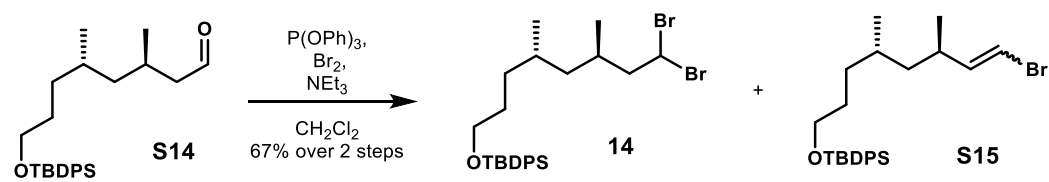

tert-Butyl(((4S,6R)-8,8-dibromo-4,6-dimethyloctyl)oxy)diphenylsilane (14):

Dibromide 14 was made according to a modified literature procedure. ${ }^{2}$

To a solution of triphenyl phosphite $\left(0.99 \mathrm{~mL}, 2.78\right.$ mmol, 1.5 equiv) in $\mathrm{CH}_{2} \mathrm{Cl}_{2}(15 \mathrm{~mL})$ at $78{ }^{\circ} \mathrm{C}$, was added bromine $(0.17 \mathrm{~mL}, 3.28 \mathrm{mmol}, 1.3$ equiv) dropwise while stirring vigorously to maintain a colorless solution. Then $\mathrm{NEt}_{3}(1.05 \mathrm{~mL}, 7.56 \mathrm{mmol}, 3.0$ equiv) was added, followed by aldehyde $\mathbf{S} 14$ (1.03 g, $2.52 \mathrm{mmol}, 1.0$ equiv) in $\mathrm{CH}_{2} \mathrm{Cl}_{2}(10 \mathrm{~mL})$. The faint orange solution was stirred at $-78{ }^{\circ} \mathrm{C}$ for $2 \mathrm{~h}$ and then warmed to rt and stirred for another $4 \mathrm{~h}$ The crude mixture was 
concentrated in vacuo and purified by flash chromatography $(0 \rightarrow 10 \%$ EtOAc in hex $)$ to afford dibromide 14 (1.68 g, 67\% yield over 2 steps) as a yellow oil in a 10:1:1 inconsequential mixture of geminal di-bromo alkane and cis and trans vinyl bromide diastereomers S15.

${ }^{1} \mathrm{H}$ NMR $\left(500 \mathrm{MHz}, \mathrm{CDCl}_{3}\right) \delta 7.75$ (ddd, $\left.J=8.0,1.5,0.4 \mathrm{~Hz}, 4 \mathrm{H}\right), 7.52-7.43(\mathrm{~m}, 6 \mathrm{H}), 5.79$ (dd, $J=8.2,6.1 \mathrm{~Hz}, 1 \mathrm{H}), 3.72(\mathrm{t}, \mathrm{J}=6.5 \mathrm{~Hz}, 2 \mathrm{H}), 2.44$ (ddd, $J=14.2,8.2,5.8 \mathrm{~Hz}, 1 \mathrm{H}$ ), 2.30, (ddd, $J=14.5,8.0,6.5 \mathrm{~Hz}), 1.89(\mathrm{tt}, J=12.9,6.4 \mathrm{~Hz}, 1 \mathrm{H}), 1.70-1.51(\mathrm{~m}, 4 \mathrm{H}), 1.40(\mathrm{~m}, 1 \mathrm{H}), 1.32-$ $1.23(\mathrm{~m}, 1 \mathrm{H}), 1.22-1.16(\mathrm{~m}, 1 \mathrm{H}), 1.13(\mathrm{~s}, 9 \mathrm{H}), 0.93(\mathrm{~d}, J=6.6 \mathrm{~Hz}, 3 \mathrm{H}), 0.92(\mathrm{~d}, J=6.5 \mathrm{~Hz}, 3 \mathrm{H})$.

${ }^{13} \mathrm{C}\left\{{ }^{1} \mathrm{H}\right\}$ NMR $\left(126 \mathrm{MHz}, \mathrm{CDCl}_{3}\right) \delta 135.7,134.3,129.7,129.6,129.6,127.64,64.4,53.7,45.1$, $43.7,33.8,30.2,30.1,29.8,27.1,19.5,19.4,18.6$.

HRMS (Cl-TOF) m/z: Calcd for $\mathrm{C}_{26} \mathrm{H}_{38} \mathrm{Br}_{2} \mathrm{OSiH}^{+}[\mathrm{M}+\mathrm{H}]^{+}$555.1119; Found 555.1132.

\section{Diagnostic data for}

\section{(((4S,6R)-8-bromo-4,6-dimethyloct-7-en-1-yl)oxy)(tert-butyl)diphenylsilane (S15):}

${ }^{1} \mathrm{H}$ NMR $\left(500 \mathrm{MHz}, \mathrm{CDCl}_{3}\right) \delta 6.13(\mathrm{dd}, J=13.5,8.2 \mathrm{~Hz}, 1 \mathrm{H}$, trans), $6.12(\mathrm{dd}, J=6.9,0.8 \mathrm{~Hz}$, $1 \mathrm{H}$, cis), 6.04 (dd, $J=13.5,0.7 \mathrm{~Hz}$, trans), 5.92, (dd, $J=9.2,6.9 \mathrm{~Hz}, 1 \mathrm{H} \mathrm{cis}$ ), 1.04 (d, $J=3.0 \mathrm{~Hz}$, $3 \mathrm{H}), 1.02(\mathrm{~d}, \mathrm{~J}=3.0 \mathrm{~Hz}, 3 \mathrm{H})$

${ }^{13} \mathrm{C}\left\{{ }^{1} \mathrm{H}\right\}$ NMR $\left(126 \mathrm{MHz}, \mathrm{CDCl}_{3}\right) \delta 144.34,141.28,129.5,127.62,105.85,103.05$

HRMS (Cl-TOF) m/z: Calcd for $\mathrm{C}_{26} \mathrm{H}_{37} \mathrm{BrOSiH}^{+}[\mathrm{M}+\mathrm{H}]^{+}$475.1859; Found 478.1838.

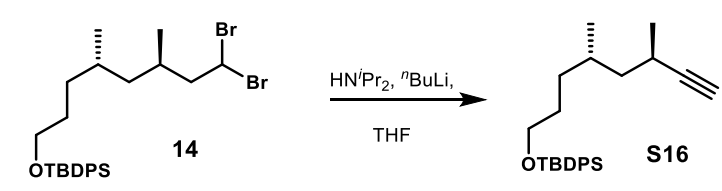

tert-Butyl(((4S,6R)-4,6-dimethyloct-7-yn-1-yl)oxy)diphenylsilane (S16):

To a solution of $\mathrm{HN}^{\mathrm{P}} \mathrm{Pr}_{2}(0.99 \mathrm{~mL}, 7.00 \mathrm{mmol}, 7.0$ equiv $)$ in $\mathrm{THF}(50 \mathrm{~mL})$ at $-78^{\circ} \mathrm{C}$ was added ${ }^{n} \mathrm{BuLi}\left(2.5 \mathrm{M}\right.$ in hex; $2.4 \mathrm{~mL}, 6.00 \mathrm{mmol}, 6.0$ equiv). The solution was stirred at $-78{ }^{\circ} \mathrm{C}$ for $5 \mathrm{~min}$, warmed to rt and stirred for $5 \mathrm{~min}$, and then cooled back to $-78{ }^{\circ} \mathrm{C}$. A solution of geminal di-bromo 14 (558 mg, $1.00 \mathrm{mmol}, 1.0$ equiv) in THF (5 mL) was added. The resulting yellow mixture was stirred at $-78{ }^{\circ} \mathrm{C}$ for $14 \mathrm{~h}$, after which it was quenched with saturated $\mathrm{NH}_{4} \mathrm{Cl}$ solution $(5 \mathrm{~mL})$. The mixture was extracted with $\mathrm{Et}_{2} \mathrm{O}(3 \times 5 \mathrm{~mL})$, then the combined organic phases were washed with brine, dried with $\mathrm{Na}_{2} \mathrm{SO}_{4}$, filtered, and concentrated in vacuo. The resulting red/orange oil was subject to the next step without further purification. An analytical aliquot was reserved for purification by flash chromatography $(0 \rightarrow 5 \%$ EtOAc in hex) to obtain alkyne S16. 
${ }^{1} \mathrm{H}$ NMR $\left(600 \mathrm{MHz}, \mathrm{CDCl}_{3}\right) \delta 7.73-7.70(\mathrm{~m}, 4 \mathrm{H}), 7.48-7.38(\mathrm{~m}, 6 \mathrm{H}), 6.69(\mathrm{t}, J=6.6 \mathrm{~Hz}$, 2H), 2.51 (ddd, $J=15.4,6.9,2.2 \mathrm{~Hz}, 1 \mathrm{H}$ ), $2.03(\mathrm{~d}, J=2.3 \mathrm{~Hz}, 1 \mathrm{H}), 1.72-1.60(\mathrm{~m}, 2 \mathrm{H}), 1.59-$ $1.53(\mathrm{~m}, 1 \mathrm{H}), 1.50-1.32(\mathrm{~m}, 3 \mathrm{H}), 1.18(\mathrm{~d}, J=6.9 \mathrm{~Hz}, 3 \mathrm{H}), 1.09(\mathrm{~s}, 9 \mathrm{H}), 0.91(\mathrm{~d}, J=6.7 \mathrm{~Hz}, 3 \mathrm{H})$.

${ }^{13} \mathrm{C}\left\{{ }^{1} \mathrm{H}\right\}$ NMR $\left(151 \mathrm{MHz}, \mathrm{CDCl}_{3}\right) \delta 135.7,134.3,129.7,127.7,89.6,68.1,64.4,44.4,32.3$, $30.5,29.9,27.0,23.5,21.2,20.0,19.4$.

HRMS (Cl-TOF) m/z: Calcd for $\mathrm{C}_{26} \mathrm{H}_{36} \mathrm{OSiH}^{+}[\mathrm{M}+\mathrm{H}]^{+}$393.2614; Found 393.2630.

$[\alpha]^{22}=-21.6\left(c=1.0, \mathrm{CHCl}_{3}\right)$.
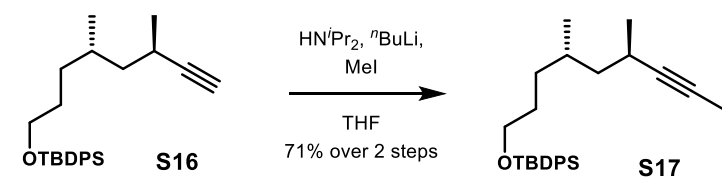

\section{tert-Butyl(((4S,6R)-4,6-dimethylnon-7-yn-1-yl)oxy)diphenylsilane (S17):}

To a solution of $\mathrm{HN}^{\prime} \mathrm{Pr}_{2}\left(0.42 \mathrm{~mL}, 3.00 \mathrm{mmol}, 3.0\right.$ equiv) in $\mathrm{THF}(5 \mathrm{~mL})$ at $-78{ }^{\circ} \mathrm{C}$ was added ${ }^{n} B u L i\left(2.5 \mathrm{M}\right.$ in hex; $0.8 \mathrm{~mL}, 2.00 \mathrm{mmol}, 2.0$ equiv). The solution was stirred at $-78{ }^{\circ} \mathrm{C}$ for $5 \mathrm{~min}$, warmed to it and stirred for $5 \mathrm{~min}$, and then cooled back to $-78^{\circ} \mathrm{C}$. A solution of terminal alkyne S16 (393 mg, $1.00 \mathrm{mmol}, 1.0$ equiv) in THF ( $5 \mathrm{~mL}$ ) was added. The resulting yellow mixture was stirred at $0{ }^{\circ} \mathrm{C}$ for $1 \mathrm{~h}$ and then cooled to $-78{ }^{\circ} \mathrm{C}$ and $\mathrm{Mel}(0.43 \mathrm{~mL}, 7.00 \mathrm{mmol}, 1.0$ equiv) was added in one portion. The solution was allowed to warm to rt overnight. The brick red solution was quenched with saturated $\mathrm{NH}_{4} \mathrm{Cl}$ solution $(5 \mathrm{~mL})$. The mixture was extracted with $\mathrm{Et}_{2} \mathrm{O}(3 \times 5 \mathrm{~mL})$, then the combined organic phases were washed with brine, dried with $\mathrm{Na}_{2} \mathrm{SO}_{4}$, filtered, and concentrated in vacuo. The crude oil was purified by flash chromatography ( $0 \rightarrow 5 \%$ EtOAc in hex) to afford internal alkyne $\mathbf{S} 17$ ( $289 \mathrm{mg}, 71 \%$ over 2 steps) as a colorless oil.

${ }^{1} \mathrm{H}$ NMR $\left(600 \mathrm{MHz}, \mathrm{CDCl}_{3}\right) \delta 7.72(\mathrm{~d}, \mathrm{~J}=6.9 \mathrm{~Hz}, 4 \mathrm{H}), 7.50-7.36(\mathrm{~m}, 6 \mathrm{H}), 3.69(\mathrm{t}, \mathrm{J}=6.6 \mathrm{~Hz}$, 2H), 2.45 (qt, J = 6.6, $2.4 \mathrm{~Hz}, 1 \mathrm{H}), 1.80(\mathrm{~d}, J=2.3 \mathrm{~Hz}, 3 \mathrm{H}), 1.69-1.60(\mathrm{~m}, 2 \mathrm{H}), 1.59-1.53(\mathrm{~m}$, $1 \mathrm{H}), 1.48-1.42(\mathrm{~m}, 1 \mathrm{H}), 1.38-1.27(\mathrm{~m}, 2 \mathrm{H}), 1.21-1.15(\mathrm{~m}, 1 \mathrm{H}), 1.13(\mathrm{~d}, J=6.8 \mathrm{~Hz}, 3 \mathrm{H}), 1.10$ $(\mathrm{s}, 9 \mathrm{H}), 0.90(\mathrm{~d}, J=6.7 \mathrm{~Hz}, 3 \mathrm{H})$.

${ }^{13} \mathrm{C}\left\{{ }^{1} \mathrm{H}\right\}$ NMR $\left(151 \mathrm{MHz}, \mathrm{CDCl}_{3}\right) \delta 135.7,134.3,129.6,127.7,89.6,84.4,75.3,64.5,44.9$, $32.4,30.5,29.9,27.0,23.6,21.6,20.0,19.4,3.6$.

HRMS (Cl-TOF) m/z: Calcd for $\mathrm{C}_{27} \mathrm{H}_{38} \mathrm{OSiH}^{+}[\mathrm{M}+\mathrm{H}]^{+}$407.2770; Found 407.2777.

$[\alpha]^{22}=-10.1\left(c=1.0, \mathrm{CHCl}_{3}\right)$. 


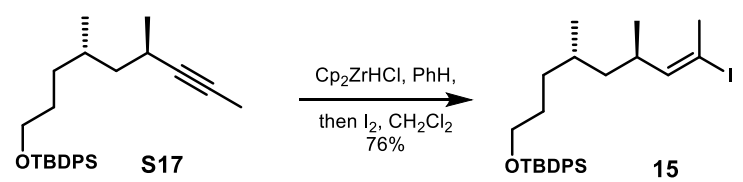

tert-Butyl(((4S,6R,E)-8-iodo-4,6-dimethylnon-7-en-1-yl)oxy)diphenylsilane (15):

$\mathrm{Cp}_{2} \mathrm{ZrHCl}$ was synthesized according to a known procedure and stored in a nitrogen filled glovebox. ${ }^{4}$

To a Schlenk flask containing $\mathrm{Cp}_{2} \mathrm{ZrHCl}(457 \mathrm{mg}, 1.77 \mathrm{mmol}, 2.5$ equiv) in benzene $(2 \mathrm{~mL})$ at rt was added a solution of alkyne $\mathbf{S} 17(289 \mathrm{mg}, 0.710 \mathrm{mmol}, 1.0$ equiv) in benzene (2 $\mathrm{mL})$. The vial containing $\mathbf{S 1 7}$ was rinsed with benzene $(2 \mathrm{~mL})$. The mixture was stirred at rt overnight. The following morning, a saturated solution of $\mathrm{I}_{2}$ in $\mathrm{CH}_{2} \mathrm{Cl}_{2}$ was added while stirring vigorously until the brown color persisted in the solution. Saturated $\mathrm{Na}_{2} \mathrm{~S}_{2} \mathrm{O}_{3}$ solution was added until the brown color dissipated to leave a cloudy white mixture. The aqueous layer was extracted with $\mathrm{Et}_{2} \mathrm{O}(6 \times 10$ $\mathrm{mL}$ ). The combined organic layers were washed with brine, dried with $\mathrm{Na}_{\mathrm{s}} \mathrm{SO}_{4}$, filtered, and concentrated in vacuo. The resulting oil was purified by flash chromatography $(0 \rightarrow 10 \%$ EtOAc in hex) to afford vinyl iodide 15 (287 $\mathrm{mg}, 76 \%)$ as a colorless oil.

${ }^{1} \mathrm{H}$ NMR $\left(500 \mathrm{MHz}, \mathrm{CDCl}_{3}\right) \delta 7.78-7.64(\mathrm{~m}, 4 \mathrm{H}), 7.55-7.33(\mathrm{~m}, 6 \mathrm{H}), 5.97(\mathrm{dd}, J=9.8,1.5$ $\mathrm{Hz}, 1 \mathrm{H}), 3.68(\mathrm{t}, J=6.6 \mathrm{~Hz}, 2 \mathrm{H}), 2.53-2.44(\mathrm{~m}, 1 \mathrm{H}), 2.36(\mathrm{~d}, J=1.5 \mathrm{~Hz}, 3 \mathrm{H}), 1.66-1.57(\mathrm{~m}$, $1 \mathrm{H}), 1.56-1.48(\mathrm{~m}, 1 \mathrm{H}), 1.46-1.34(\mathrm{~m}, 2 \mathrm{H}), 1.24-1.18(\mathrm{~m}, 1 \mathrm{H}), 1.16-1.11(\mathrm{~m}, 1 \mathrm{H}), 1.09(\mathrm{~s}$, $3 \mathrm{H}), 0.94(\mathrm{~d}, J=6.6 \mathrm{~Hz}, 3 \mathrm{H}), 0.87(\mathrm{~d}, J=6.6 \mathrm{~Hz}, 3 \mathrm{H})$.

${ }^{13} \mathrm{C}\left\{{ }^{1} \mathrm{H}\right\}$ NMR $\left(126 \mathrm{MHz}, \mathrm{CDCl}_{3}\right) \delta 147.9,135.7,134.2,134.2,129.7,129.6,127.7,127.7$, $92.2,44.5,33.4,32.9,30.3,30.0,27.8,27.1,20.4,20.1,19.3$.

HRMS (Cl-TOF) m/z: Calcd for $\mathrm{C}_{27} \mathrm{H}_{39} \mathrm{IOSiH}^{+}[\mathrm{M}+\mathrm{H}]^{+}$535.1893; Found 535.1887.

$[\alpha]^{22}=-20.2\left(c=10.0, \mathrm{CHCl}_{3}\right)$.

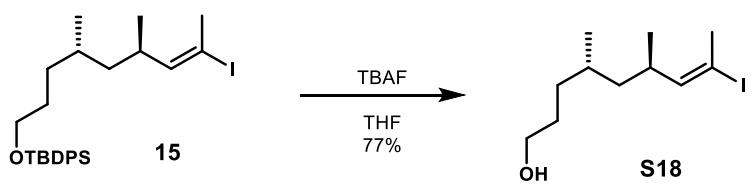

(4S,6R,E)-8-iodo-4,6-dimethylnon-7-en-1-ol (S115):

To a solution of vinyl iodide 15 (144 $\mathrm{mg}, 0.270 \mathrm{mmol}, 1.0$ equiv) in THF (2 mL) at rt was added TBAF (1.0 M in THF; $0.54 \mathrm{~mL}, 0.540 \mathrm{mmol}, 2.0$ equiv) dropwise. Upon addition of TBAF, the solution darkened from faint yellow to green and finally magenta. The solution was stirred 
overnight, after which it was quenched with saturated $\mathrm{NaHCO}_{3}(2 \mathrm{~mL})$ solution and extracted with $\mathrm{Et}_{2} \mathrm{O}(2 \times 2 \mathrm{~mL})$. The combined organic phase was washed with brine, dried with $\mathrm{MgSO}_{4}$, filtered, and concentrated in vacuo. The crude oil was purified by flash chromatography $(0 \rightarrow 10 \% \mathrm{MeOH}$ in $\mathrm{CH}_{2} \mathrm{Cl}_{2}$ ) to afford alcohol $\mathbf{S 1 8}(128 \mathrm{mg}, 62 \%)$ as a colorless oil.

${ }^{1} \mathrm{H}$ NMR $\left(500 \mathrm{MHz}, \mathrm{CDCl}_{3}\right) \delta 5.91(\mathrm{dd}, J=9.8,1.3 \mathrm{~Hz}, 1 \mathrm{H}), 3.58(\mathrm{td}, J=6.7,1.8 \mathrm{~Hz}, 2 \mathrm{H}), 2.51$ $-2.40(\mathrm{~m}, 1 \mathrm{H}), 2.35(\mathrm{~d}, J=1.6 \mathrm{~Hz}, 3 \mathrm{H}), 1.77(\mathrm{~s}, 1 \mathrm{H}), 1.63-1.53(\mathrm{~m}, 1 \mathrm{H}), 1.51-1.29(\mathrm{~m}, 2 \mathrm{H})$, $1.20(\mathrm{dt}, J=13.6,6.8 \mathrm{~Hz}, 1 \mathrm{H}), 1.13-1.04(\mathrm{~m}, 2 \mathrm{H}), 0.90(\mathrm{~d}, J=6.7 \mathrm{~Hz}, 3 \mathrm{H}), 0.85(\mathrm{~d}, J=6.7 \mathrm{~Hz}$, $3 \mathrm{H})$.

${ }^{13} \mathrm{C}\left\{{ }^{1} \mathrm{H}\right\} \operatorname{NMR}\left(126 \mathrm{MHz}, \mathrm{CDCl}_{3}\right) \delta 147.8,92.2,63.3,44.4,33.3,32.7,30.3,30.1,27.7,20.3$, 20.0 .

HRMS (ESI-TOF) m/z: Calcd for $\mathrm{C}_{11} \mathrm{H}_{21} \mathrm{OINa}^{+}[\mathrm{M}+\mathrm{Na}]^{+} 319.0535$; Found 319.0538.

$$
[\alpha]^{22}=-47.7\left(c=1.0, \mathrm{CHCl}_{3}\right) \text {. }
$$
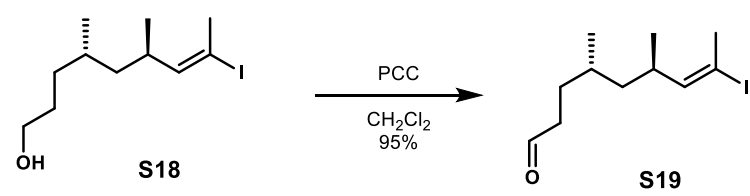

\section{$(4 S, 6 R, E)-8-i o d o-4,6-d i m e t h y l n o n-7-e n a l ~(S 19):$}

To a solution of alcohol $\mathbf{S 1 8}$ (61 mg, $0.207 \mathrm{mmol}, 1.0$ equiv) in $\mathrm{CH}_{2} \mathrm{Cl}_{2}(1 \mathrm{~mL})$ was added PCC (67 mg, $0.310 \mathrm{mmol}, 1.5$ equiv) in one portion. The mixture was stirred at $\mathrm{rt}$ for $2 \mathrm{~h}$. The crude mixture was run through a pad of silica and the cake was washed thoroughly with $\mathrm{CH}_{2} \mathrm{Cl}_{2}$. The resulting aldehyde $\mathbf{S 1 9}$ was taken on to the next step without further purification (57 $\mathrm{mg}, 95 \%$ ) as a clear, colorless oil.

${ }^{1} \mathrm{H}$ NMR $\left(500 \mathrm{MHz}, \mathrm{CDCl}_{3}\right) \delta 9.77(\mathrm{~s}, 1 \mathrm{H}), 5.94(\mathrm{dd}, J=9.8,1.3 \mathrm{~Hz}, 1 \mathrm{H}), 2.53-2.39(\mathrm{~m}, 3 \mathrm{H})$, $2.38(\mathrm{~d}, J=1.4 \mathrm{~Hz}, 3 \mathrm{H}), 1.71-1.63(\mathrm{~m}, 1 \mathrm{H}), 1.49-1.42(\mathrm{~m}, 1 \mathrm{H}), 1.38$ (ddd, $J=11.5,8.1,2.8$ $\mathrm{Hz}, 1 \mathrm{H}), 1.21(\mathrm{dd}, J=13.6,6.8 \mathrm{~Hz}, 1 \mathrm{H}), 1.18-1.11(\mathrm{~m}, 1 \mathrm{H}), 0.93(\mathrm{~d}, J=6.6 \mathrm{~Hz}, 3 \mathrm{H}), 0.88(\mathrm{~d}, J$ $=6.5 \mathrm{~Hz}, 3 \mathrm{H})$.

${ }^{13} \mathrm{C}\left\{{ }^{1} \mathrm{H}\right\}$ NMR $\left(126 \mathrm{MHz}, \mathrm{CDCl}_{3}\right) \delta 202.8,147.5,92.5,44.2,41.7,33.3,30.2,28.7,27.8,20.3$, 19.7 .

HRMS (Cl-TOF) m/z: Calcd for $\mathrm{C}_{11} \mathrm{H}_{19} \mathrm{IO}^{+}[\mathrm{M}]^{+}$294.0481; Found 294.0484.

$$
[\alpha]^{22}=-47.7\left(c=1.0, \mathrm{CHCl}_{3}\right) \text {. }
$$




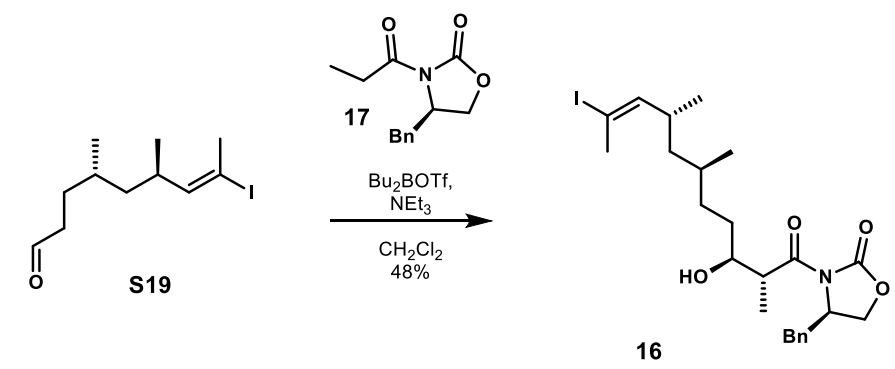

(R)-4-benzyl-3-((2R,3S,6S,8R,E)-3-hydroxy-10-iodo-2,6,8-trimethylundec-9enoyl)oxazolidin-2-one (16):

Oxazolidinone 17 was made according to a known literature procedure. ${ }^{10}$

To a solution of acylated oxazolidinone 17 (49 mg, $0.211 \mathrm{mmol}, 1.1$ equiv) in $\mathrm{CH}_{2} \mathrm{Cl}_{2}(0.5 \mathrm{~mL})$ at $0{ }^{\circ} \mathrm{C}$ was added freshly distilled $\mathrm{Bu}_{2} \mathrm{BOTf}^{11}\left(0.07 \mathrm{~mL}, 0.250 \mathrm{mmol}, 1.3\right.$ equiv), then $\mathrm{Et}_{3} \mathrm{~N}(0.04$ $\mathrm{mL}, 0.269 \mathrm{mmol}, 1.4$ equiv). The solution turned light green. The reaction was cooled to $-78{ }^{\circ} \mathrm{C}$ and a solution of aldehyde $\mathbf{S} 19$ (57 mg, $0.192 \mathrm{mmol}, 1.0$ equiv) in $\mathrm{CH}_{2} \mathrm{Cl}_{2}(1.0 \mathrm{~mL})$ was added. The mixture was stirred for $30 \mathrm{~min}$ at $-78{ }^{\circ} \mathrm{C}$ and then warmed to $0{ }^{\circ} \mathrm{C}$ and stirred for another 1.5 h. While stirring at $0{ }^{\circ} \mathrm{C}$, a solution of phosphate buffer $(1 \mathrm{~mL}, \mathrm{pH} 7)$ and $\mathrm{MeOH}(2 \mathrm{~mL})$ was added, followed by a solution of $30 \%$ aq. $\mathrm{H}_{2} \mathrm{O}_{2}(2 \mathrm{~mL})$ and $\mathrm{MeOH}(1 \mathrm{~mL})$. The mixture was stirred for 30 min. The aqueous layer was diluted with $\mathrm{H}_{2} \mathrm{O}(1 \mathrm{~mL})$ and the organic layer extracted thoroughly with $\mathrm{Et}_{2} \mathrm{O}(5 \times 10 \mathrm{~mL})$. The combined organic phase was washed with brine, dried with $\mathrm{Na}_{2} \mathrm{SO}_{4}$, filtered, and concentrated in vacuo. The crude mixture was purified by flash chromatography $(0 \rightarrow 50 \%$ EtOAc in hex) to afford aldol adduct 16 as a colorless oil ( $49 \mathrm{mg}, 48 \%)$.

${ }^{1} \mathrm{H}$ NMR $\left(600 \mathrm{MHz}, \mathrm{CDCl}_{3}\right) \delta 7.32(\mathrm{t}, J=7.4 \mathrm{~Hz}, 2 \mathrm{H}), 7.29-7.24(\mathrm{~m}, 1 \mathrm{H}), 7.18(\mathrm{~d}, J=7.3 \mathrm{~Hz}$, $2 \mathrm{H}), 5.91(\mathrm{dd}, J=9.8,1.2 \mathrm{~Hz}, 1 \mathrm{H}), 4.72-4.67(\mathrm{~m}, 1 \mathrm{H}), 4.24-4.16(\mathrm{~m}, 2 \mathrm{H}), 3.88(\mathrm{dd}, J=4.9$, $3.1 \mathrm{~Hz}, 1 \mathrm{H}$ ), 3.74 (qd, J = 7.0, $2.7 \mathrm{~Hz}, 1 \mathrm{H}), 3.23$ (dd, $J=13.4,3.1 \mathrm{~Hz}, 1 \mathrm{H}), 2.90(\mathrm{~s}, 1 \mathrm{H}), 2.78$ (dd, $J=13.4,9.4 \mathrm{~Hz}, 1 \mathrm{H}), 2.52-2.43(\mathrm{~m}, 1 \mathrm{H}), 2.36(\mathrm{~d}, J=1.2 \mathrm{~Hz}, 3 \mathrm{H}), 1.61-1.52(\mathrm{~m}, 1 \mathrm{H}), 1.45-$ $1.38(\mathrm{~m}, 1 \mathrm{H}), 1.37-1.29(\mathrm{~m}, 2 \mathrm{H}), 1.24(\mathrm{~d}, J=7.1 \mathrm{~Hz}, 3 \mathrm{H}), 1.22-1.16(\mathrm{~m}, 2 \mathrm{H}), 1.13-1.07(\mathrm{~m}$, $1 \mathrm{H}), 0.90(\mathrm{~d}, J=6.6 \mathrm{~Hz}, 3 \mathrm{H}), 0.85(\mathrm{~d}, J=6.6 \mathrm{~Hz}, 3 \mathrm{H})$.

${ }^{13} \mathrm{C}\left\{{ }^{1} \mathrm{H}\right\}$ NMR $\left(151 \mathrm{MHz}, \mathrm{CDCl}_{3}\right) \delta 177.67,153.1,147.8,135.1,129.5,129.1,127.5,92.3$, $71.7,66.3,55.2,44.4,42.2,37.87,33.3,32.8,31.2,30.3,27.8,20.4,20.0,10.5$.

HRMS (TOF-ESI) m/z: Calcd for $\mathrm{C}_{24} \mathrm{H}_{34} \mathrm{INO}_{4} \mathrm{Na}^{+}[\mathrm{M}+\mathrm{Na}]^{+}$550.1430; Found 550.1436.

$[\alpha]^{22}=-46.8\left(c=1.0, \mathrm{CHCl}_{3}\right)$. 


\section{Final Macrocyclization:}

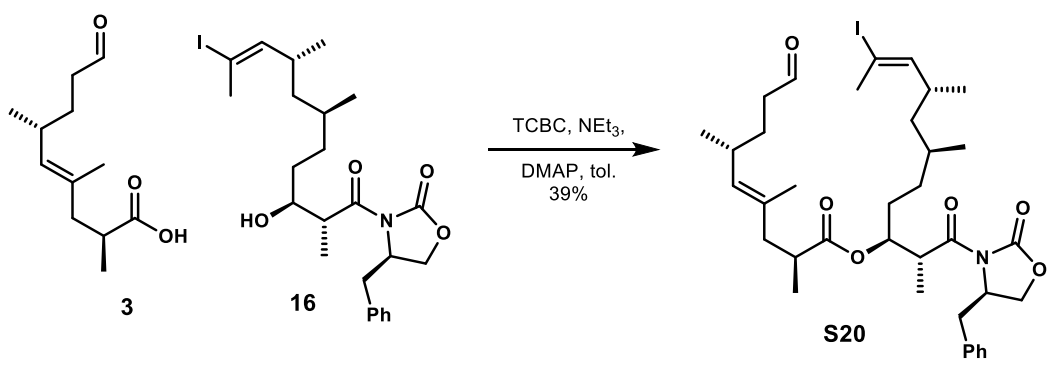

$(2 R, 3 S, 6 S, 8 R, E)-1-((R)-4-B e n z y l-2-0 x o o x a z o l i d i n-3-y l)-10$-iodo-2,6,8-trimethyl-1-oxoundec9-en-3-yl (2S,6R,E)-2,4,6-trimethyl-9-oxonon-4-enoate (S20):

To a solution of carboxylic acid $3(24 \mathrm{mg}, 0.112 \mathrm{mmol}, 1.2$ equiv) in toluene $(0.5 \mathrm{~mL})$ was added 2,4,6-trichlorobenzoyl chloride ( $20 \mu \mathrm{L}, 0.139 \mathrm{mmol}, 1.5$ equiv) followed by $\mathrm{NEt}_{3}(20 \mu \mathrm{L}$, $0.139 \mathrm{mmol}, 1.5$ equiv). The solution was stirred for $30 \mathrm{~min}$ then alcohol 16 (49 $\mathrm{mg}, 0.0929 \mathrm{mmol}$, 1.0 equiv) in toluene $(0.5 \mathrm{~mL}$ ) was added followed by DMAP (34 mg, $0.279 \mathrm{mmol}, 3.0$ equiv). After stirring for $20 \mathrm{~min}$ at $\mathrm{rt}$, the reaction was quenched with saturated $\mathrm{NH}_{4} \mathrm{Cl}$ solution and then extracted with $\mathrm{Et}_{2} \mathrm{O}(2 \times 5 \mathrm{~mL})$. The combined organic phase was washed with brine, dried with $\mathrm{MgSO}_{4}$, filtered, and concentrated in vacuo. The resulting residue was purified via flash chromatography $(0 \rightarrow 35 \%$ EtOAc in hex) to obtain ester $\mathbf{S 2 0}$ as a clear oil $(26 \mathbf{m g}, 39 \%)$.

${ }^{1} \mathrm{H}$ NMR $\left(500 \mathrm{MHz}, \mathrm{CDCl}_{3}\right): \delta 9.75(\mathrm{~s}, 1 \mathrm{H}), 7.32(\mathrm{t}, J=7.3 \mathrm{~Hz}, 2 \mathrm{H}), 7.29-7.26(\mathrm{~m}, 1 \mathrm{H}), 7.19$ $(\mathrm{d}, J=7.3 \mathrm{~Hz}, 2 \mathrm{H}), 5.93(\mathrm{~d}, J=9.8 \mathrm{~Hz}, 1 \mathrm{H}), 5.18(\mathrm{dt}, J=8.4,4.3 \mathrm{~Hz}, 1 \mathrm{H}), 4.92(\mathrm{~d}, J=9.5 \mathrm{~Hz}$, $1 \mathrm{H}), 4.57-4.49(\mathrm{~m}, 1 \mathrm{H}), 4.28(\mathrm{t}, J=8.3 \mathrm{~Hz}, 1 \mathrm{H}), 4.15(\mathrm{dd}, J=8.9,2.0 \mathrm{~Hz}, 1 \mathrm{H}), 4.00-3.92(\mathrm{~m}$, 1H), $3.26(\mathrm{dd}, J=13.4,2.9 \mathrm{~Hz}, 1 \mathrm{H}), 2.77(\mathrm{dd}, J=13.4,9.7 \mathrm{~Hz}, 1 \mathrm{H}), 2.61-2.53(\mathrm{~m}, 1 \mathrm{H}), 2.52-$ $2.41(\mathrm{~m}, 2 \mathrm{H}), 2.41-2.32(\mathrm{~m}, 6 \mathrm{H}), 1.98(\mathrm{dd}, J=13.6,9.3 \mathrm{~Hz}, 1 \mathrm{H}), 1.73-1.62(\mathrm{~m}, 2 \mathrm{H}), 1.60-$ $1.55(\mathrm{~m}, 4 \mathrm{H}), 1.56-1.46(\mathrm{~m}, 2 \mathrm{H}), 1.45-1.36(\mathrm{~m}, 1 \mathrm{H}), 1.29(\mathrm{ddd}, J=12.8,9.9,3.3 \mathrm{~Hz}, 1 \mathrm{H}), 1.22$ $-1.15(\mathrm{~m}, 4 \mathrm{H}), 1.12-1.06(\mathrm{~m}, 4 \mathrm{H}), 0.95(\mathrm{~d}, J=6.6 \mathrm{~Hz}, 3 \mathrm{H}), 0.91(\mathrm{~d}, J=6.6 \mathrm{~Hz}, 3 \mathrm{H}), 0.86(\mathrm{~d}, J$ $=6.6 \mathrm{~Hz}, 3 \mathrm{H})$.

${ }^{13} \mathrm{C}\left\{{ }^{1} \mathrm{H}\right\}$ NMR $\left(126 \mathrm{MHz}, \mathrm{CDCl}_{3}\right) \delta 202.8,176.3,174.1,153.8,147.8,135.6,132.9,132.1$, 129.6, 129.1, 127.4, 92.4, 73.4, 66.5, 56.0, 44.4, 43.8, 42.3, 41.5, 38.1, 38.0, 33.4, 32.7, 32.2, $30.4,30.0,29.8,27.8,21.3,20.2,19.8,16.5,16.0,10.1$.

HRMS (ESI-TOF) m/z: Calcd for $\mathrm{C}_{36} \mathrm{H}_{52} \mathrm{NO}_{6} \mathrm{INa}^{+}[\mathrm{M}+\mathrm{Na}]^{+}$744.2737; Found 744.2716.

$$
[\alpha]^{22} \mathrm{D}=-37.3\left(c=1.0, \mathrm{CHCl}_{3}\right) \text {. }
$$



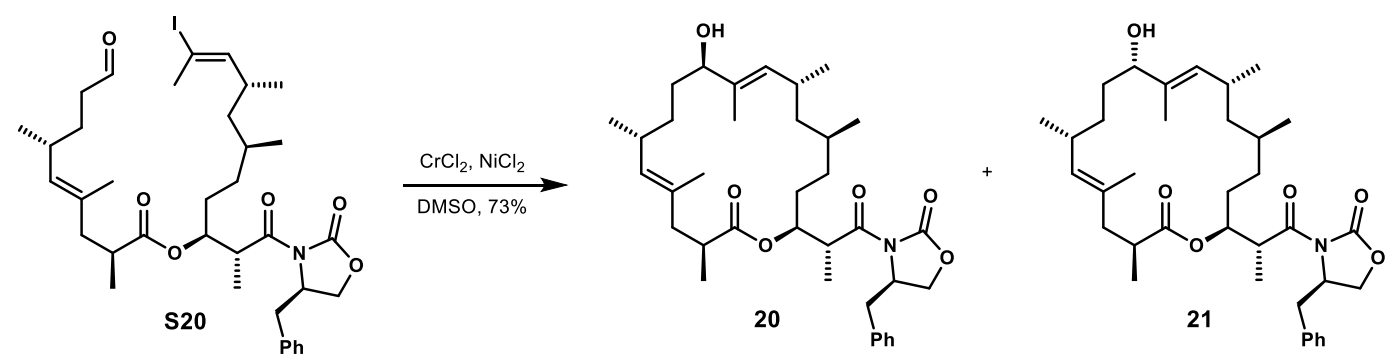

$(R)-4-$ benzyl-3-((R)-2-((2S,5S,7R,8E,10R,13R,14E,17S)-10-hydroxy-5,7,9,13,15,17hexamethyl-18-oxooxacyclooctadeca-8,14-dien-2-yl)propanoyl)oxazolidin-2-one (20) and $(R)-4-b e n z y l-3-((R)-2-((2 S, 5 S, 7 R, 8 E, 10 S, 13 R, 14 E, 17 S)-10$-hydroxy-5,7,9,13,15,17hexamethyl-18-oxooxacyclooctadeca-8,14-dien-2-yl)propanoyl)oxazolidin-2-one (21):

To a solution of aldehyde $\mathbf{S 2 0}$ (32 $\mathrm{mg}, 0.0444 \mathrm{mmol}, 1.0$ equiv) in DMSO (4.4 mL), in a nitrogen filled glovebox, was added $\mathrm{CrCl}_{2}$ (109 $\mathrm{mg}, 0.888 \mathrm{mmol}, 20$ equiv) followed by $\mathrm{NiCl}_{2}$ (1 $\mathrm{mg}, 0.00772 \mathrm{mmol}, 0.17$ equiv). The solution was stirred overnight at it in the glovebox, and removed the next morning. The black solution was diluted with $\mathrm{H}_{2} \mathrm{O}(15 \mathrm{~mL})$ and extracted with EtOAc $(5 \times 5 \mathrm{~mL})$. The combined organic phase was washed with $\mathrm{H}_{2} \mathrm{O}(5 \times 5 \mathrm{~mL})$ and brine, dried with $\mathrm{Na}_{2} \mathrm{SO}_{4}$, filtered, and concentrated in vacuo. The resulting residue was purified via flash chromatography (20\% EtOAc in hex) to obtain the diastereomers as a mixture 1.1:1 dr of $\alpha: \beta$ face alcohol $(19 \mathrm{mg}, 73 \%)$. The diastereomers were separated via a second flash column $(20 \% \rightarrow$ $30 \%$ EtOAc in hex; $500 \mathrm{wt} \%$ of silica to mixture mass) to obtain $\beta$-face alcohol 20 (6 mg, 23\%) as a white amorphous solid and $\alpha$-face alcohol 21 (8 $\mathrm{mg}, 31 \%)$ white amorphous solid.

\section{$\beta$-face alcohol 20:}

$$
\mathbf{R}_{\mathbf{f}}=0.34 \text { (7/3; hex/EtOAc) }
$$

${ }^{1} \mathrm{H}$ NMR $\left(600 \mathrm{MHz}, \mathrm{CDCl}_{3}\right): \delta 7.32(\mathrm{t}, J=7.3 \mathrm{~Hz}, 2 \mathrm{H}), 7.29-7.26(\mathrm{~m}, 1 \mathrm{H}), 7.20(\mathrm{~d}, J=7.0$ $\mathrm{Hz}, 2 \mathrm{H}), 5.25-5.21(\mathrm{~m}, 1 \mathrm{H}), 5.12(\mathrm{~d}, J=8.8 \mathrm{~Hz}, 1 \mathrm{H}), 4.71(\mathrm{~d}, J=8.8 \mathrm{~Hz}, 1 \mathrm{H}), 4.59-4.54(\mathrm{~m}$, $1 \mathrm{H}), 4.26(\mathrm{t}, J=8.3 \mathrm{~Hz}, 1 \mathrm{H}), 4.17(\mathrm{dd}, J=9.0,2.3 \mathrm{~Hz}, 1 \mathrm{H}), 4.03-3.98(\mathrm{~m}, 1 \mathrm{H}), 3.86-3.82(\mathrm{~m}$, 1H), 3.26 (dd, $J=13.4,3.2 \mathrm{~Hz}, 1 \mathrm{H}$ ), 2.77 (dd, $J=13.4,9.7 \mathrm{~Hz}, 1 \mathrm{H}$ ), 2.64 (ddd, $J=11.6,7.0,3.1$ $\mathrm{Hz}, 1 \mathrm{H}$ ), 2.52 (dt, $J=14.8,7.4 \mathrm{~Hz}, 1 \mathrm{H}), 2.27(\mathrm{dd}, J=14.3,11.8 \mathrm{~Hz}, 1 \mathrm{H}), 2.23-2.17(\mathrm{~m}, 1 \mathrm{H}), 2.02$ $(\mathrm{dd}, J=14.5,2.4 \mathrm{~Hz}, 1 \mathrm{H}), 1.68-1.62(\mathrm{~m}, 1 \mathrm{H}), 1.60-1.58(\mathrm{~m}, 6 \mathrm{H}), 1.43-1.35(\mathrm{~m}, 2 \mathrm{H}), 1.35-$ $1.29(\mathrm{~m}, 2 \mathrm{H}), 1.27-1.25(\mathrm{~m}, 2 \mathrm{H}), 1.27-1.22(\mathrm{~m}, 5 \mathrm{H}), 1.21(\mathrm{~d}, J=6.9 \mathrm{~Hz}, 3 \mathrm{H}), 1.11-1.03(\mathrm{~m}$, $1 \mathrm{H}), 0.95(\mathrm{~d}, J=6.7 \mathrm{~Hz}, 3 \mathrm{H}), 0.90-0.85(\mathrm{~m}, 5 \mathrm{H}), 0.83(\mathrm{~d}, J=6.7 \mathrm{~Hz}, 3 \mathrm{H})$.

${ }^{13} \mathrm{C}\left\{{ }^{1} \mathrm{H}\right\}$ NMR $\left(151 \mathrm{MHz}, \mathrm{CDCl}_{3}\right) \delta 174.8,174.2,153.6,135.6,135.5,134.8,133.2,132.1$, 129.6, 129.1, 127.5, 79.5, 73.3, 66.5, 55.9, 45.2, 43.8, 40.6, 38.0, 33.6, 32.7, 32.0, 30.5, 30.4, $29.9,29.6,29.4,21.8,21.6,21.3,18.7,17.0,11.4,10.0$. 
HRMS (ESI-TOF) m/z: Calcd for $\mathrm{C}_{36} \mathrm{H}_{53} \mathrm{NO}_{6} \mathrm{Na}^{+}[\mathrm{M}+\mathrm{Na}]^{+}$618.3771; Found 618.3763.

$[\alpha]^{22}=-12.4\left(c=0.59, \mathrm{CHCl}_{3}\right)$.

\section{a-face alcohol 21:}

$\mathbf{R}_{\mathbf{f}}=0.40(7 / 3 ;$ hex/EtOAc)

${ }^{1} \mathrm{H}$ NMR $\left(600 \mathrm{MHz}, \mathrm{CDCl}_{3}\right) \delta 7.32(\mathrm{t}, J=7.3 \mathrm{~Hz}, 2 \mathrm{H}), 7.27(\mathrm{~d}, J=7.3 \mathrm{~Hz}, 1 \mathrm{H}), 7.20$ (d, J = 7.1 $\mathrm{Hz}, 2 \mathrm{H}), 5.28-5.25(\mathrm{~m}, 2 \mathrm{H}), 4.70(\mathrm{~d}, J=8.9 \mathrm{~Hz}, 1 \mathrm{H}), 4.61-4.56(\mathrm{~m}, 1 \mathrm{H}), 4.26(\mathrm{t}, J=8.3 \mathrm{~Hz}$, $1 \mathrm{H}), 4.17(\mathrm{dd}, \mathrm{J}=9.0,2.2 \mathrm{~Hz}, 1 \mathrm{H}), 4.13-4.11(\mathrm{~m}, 1 \mathrm{H}), 4.04-3.99(\mathrm{~m}, 1 \mathrm{H}), 3.26(\mathrm{dd}, \mathrm{J}=13.4$, 3.1 Hz, 1H), 2.77 (dd, $J=13.4,9.7 \mathrm{~Hz}, 1 \mathrm{H}), 2.65$ (ddd, $J=11.7,7.0,3.1 \mathrm{~Hz}, 1 \mathrm{H}), 2.60-2.53(\mathrm{~m}$, $1 \mathrm{H}), 2.28-2.22(\mathrm{~m}, 1 \mathrm{H}), 2.21-2.14(\mathrm{~m}, 1 \mathrm{H}), 2.02(\mathrm{dd}, J=13.9,2.2 \mathrm{~Hz}, 1 \mathrm{H}), 1.77-1.65(\mathrm{~m}$, $2 \mathrm{H}), 1.59(\mathrm{~s}, 3 \mathrm{H}), 1.54(\mathrm{~s}, 3 \mathrm{H}), 1.48-1.40(\mathrm{~m}, 2 \mathrm{H}), 1.36-1.30(\mathrm{~m}, 3 \mathrm{H}), 1.29-1.26(\mathrm{~m}, 2 \mathrm{H}), 1.24$ $(\mathrm{d}, J=6.9 \mathrm{~Hz}, 3 \mathrm{H}), 1.21(\mathrm{~d}, J=6.9 \mathrm{~Hz}, 3 \mathrm{H}), 1.11-1.04(\mathrm{~m}, 2 \mathrm{H}), 0.95(\mathrm{~d}, J=6.7 \mathrm{~Hz}, 3 \mathrm{H}), 0.86$ (d, $J=6.8 \mathrm{~Hz}, 3 \mathrm{H}), 0.84(\mathrm{~d}, J=6.7 \mathrm{~Hz}, 3 \mathrm{H})$.

${ }^{13} \mathrm{C}\left\{{ }^{1} \mathrm{H}\right\}$ NMR $\left(151 \mathrm{MHz}, \mathrm{CDCl}_{3}\right) \delta 174.7,174.3,153.6,135.5,135.4,133.6,132.1,129.8$, $129.6,129.1,127.5,74.5,73.4,66.5,55.9,45.5,44.3,40.8,40.7,38.0,32.4,30.5,30.3,30.3$, $29.7,29.5,29.3,22.0,22.0,21.3,18.7,16.8,15.1,11.8$.

HRMS (ESI-TOF) m/z: Calcd for $\mathrm{C}_{36} \mathrm{H}_{53} \mathrm{NO}_{6} \mathrm{Na}^{+}[\mathrm{M}+\mathrm{Na}]^{+}$618.3771; Found 618.3780.

$[\alpha]^{22}=-19.8\left(c=1.0, \mathrm{CHCl}_{3}\right)$.
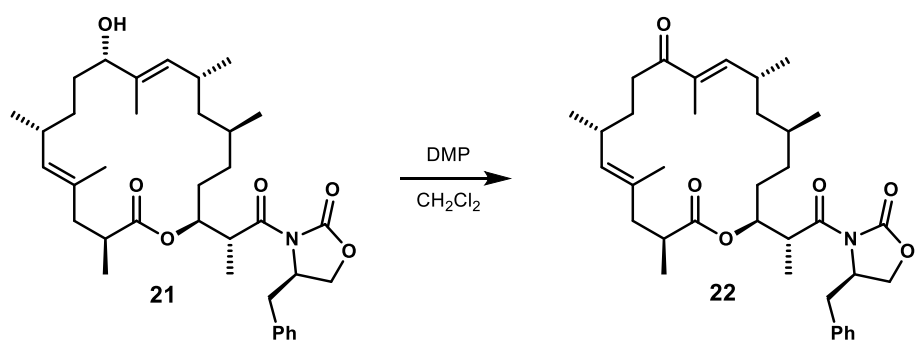

(3S,5E,7R,11E,13R,15S,18S)-18-((R)-1-((R)-4-benzyl-2-oxooxazolidin-3-yl)-1-oxopropan-2yl)-3,5,7,11,13,15-hexamethyloxacyclooctadeca-5,11-diene-2,10-dione (22):

To a solution of alcohol 21 (4.9 mg, 0.008224 mmol, 1.0 equiv) in $\mathrm{CH}_{2} \mathrm{Cl}_{2}(0.10 \mathrm{~mL}$ ) at rt was added Martin's reagent ( $5 \mathrm{mg}, 0.0126,1.5$ equiv) and the solution was stirred for $1.5 \mathrm{~h}$ at rt. The slurry was concentrated in vacuo and to the resulting residue was added $\mathrm{Et}_{2} \mathrm{O}(3 \mathrm{~mL})$. The solution was stirred for $5 \mathrm{~min}$ and then filtered and concentrated in vacuo. The resulting residue was taken 
on to the next step without further purification.

HRMS (ESI-TOF) m/z: Calcd for $\mathrm{C}_{36} \mathrm{H}_{51} \mathrm{NO}_{6} \mathrm{Na}^{+}[\mathrm{M}+\mathrm{Na}]^{+}$616.3614; Found 616.3602 .
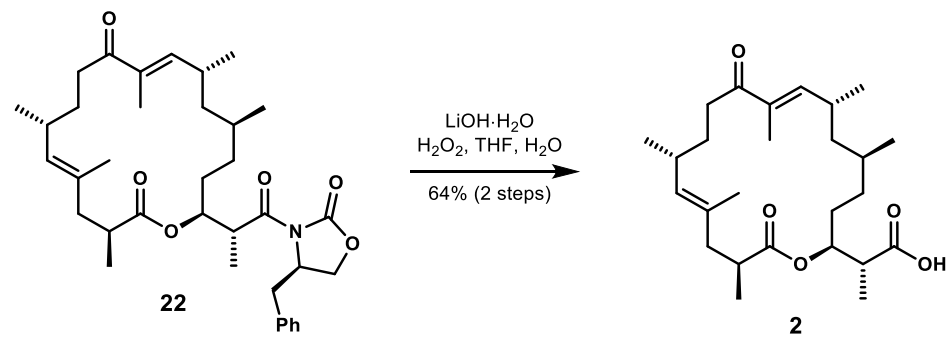

\section{Strasseriolide A (2):}

To a solution of ketone $22\left(4.9 \mathrm{mg}, 0.008224 \mathrm{mmol}, 1.0\right.$ equiv) in THF $(0.1 \mathrm{~mL})$ and $\mathrm{H}_{2} \mathrm{O}(0.5$ $\mathrm{mL}$ ) was added $\mathrm{LiOH} \cdot \mathrm{H}_{2} \mathrm{O}(2.0 \mathrm{mg})$ followed by $30 \%$ aq. $\mathrm{H}_{2} \mathrm{O}_{2}$ solution $(10 \mu \mathrm{L})$. The solution was stirred for $40 \mathrm{~min}$ and then quenched with $3 \mathrm{M} \mathrm{HCl}$. The solution was extracted with EtOAc $(3 \times 3$ $\mathrm{mL}$ ). The combined organic phase was washed with brine, dried with $\mathrm{Na}_{2} \mathrm{SO}_{4}$, filtered, and concentrated in vacuo. The resulting residue was purified via prep TLC (50/50/1 EtOAc/hex/AcOH) to obtain strasseriolide $\mathbf{A}(2.3 \mathrm{mg}, 64 \%$ over 2 steps $)$ as a white amorphous solid.

${ }^{1} \mathrm{H}$ NMR $\left(600 \mathrm{MHz}, \mathrm{CD}_{3} \mathrm{OD}\right) \delta 6.60(\mathrm{~d}, J=9.2 \mathrm{~Hz}, 1 \mathrm{H}), 5.14-5.09(\mathrm{~m}, 1 \mathrm{H}), 4.79(\mathrm{~d}, J=9.3$ $\mathrm{Hz}, 1 \mathrm{H}$ ), 2.99 (ddd, $J=15.5,7.7,4.5 \mathrm{~Hz}, 1 \mathrm{H}), 2.86-2.77(\mathrm{~m}, 1 \mathrm{H}), 2.72-2.66(\mathrm{~m}, 1 \mathrm{H}), 2.58$ (ddd, $J=10.5,7.1,3.2 \mathrm{~Hz}, 1 \mathrm{H}), 2.43-2.34(\mathrm{~m}, 2 \mathrm{H}), 2.20(\mathrm{dd}, J=14.2,11.6 \mathrm{~Hz}, 1 \mathrm{H}), 2.00(\mathrm{dd}, J=$ 14.4, 2.7 Hz, 1H), $1.87-1.79(\mathrm{~m}, 2 \mathrm{H}), 1.75(\mathrm{~s}, 3 \mathrm{H}), 1.71-1.66(\mathrm{~m}, 1 \mathrm{H}), 1.61-1.55(\mathrm{~m}, 3 \mathrm{H})$, $1.53(\mathrm{~s}, 3 \mathrm{H}), 1.51-1.40(\mathrm{~m}, 3 \mathrm{H}), 1.18(\mathrm{~d}, J=6.9 \mathrm{~Hz}, 3 \mathrm{H}), 1.13-1.07(\mathrm{~m}, 4 \mathrm{H}), 1.03(\mathrm{~d}, J=6.8$ $\mathrm{Hz}, 3 \mathrm{H}), 0.94(\mathrm{~d}, J=6.6 \mathrm{~Hz}, 3 \mathrm{H}), 0.90(\mathrm{~d}, J=6.8 \mathrm{~Hz}, 3 \mathrm{H})$.

${ }^{13} \mathrm{C}\left\{{ }^{1} \mathrm{H}\right\}$ NMR $\left(151 \mathrm{MHz}, \mathrm{CDCl}_{3}\right) \delta 205.5,177.5176 .9,150.8,137.1,134.2,133.0,75.5,45.9$, 44.6, 43.1, 42.7, 36.9, 34.9, 33.1, 31.9, 31.7, 30.7, 30.3, 22.2, 21.3, 20.9, 19.2, 17.8, 13.8, 11.8. $\left({ }^{13} \mathrm{C}\right.$ NMR contains $\sim 10$ equiv of acetic acid- $\mathrm{D}_{4}$ to resolve carboxylic acid peak).

HRMS (ESI-TOF) m/z: Calcd for $\mathrm{C}_{26} \mathrm{H}_{42} \mathrm{O}_{5} \mathrm{Na}^{+}[\mathrm{M}+\mathrm{Na}]^{+}$457.2930; Found 457.2919.

$[\alpha]^{22} \mathrm{D}=+6.3\left(c=1.0, \mathrm{CH}_{3} \mathrm{OH}\right)$. Literature reported: $[\alpha]^{25}=+8.0\left(c=0.45, \mathrm{CH}_{3} \mathrm{OH}\right) \cdot{ }^{12}$ 


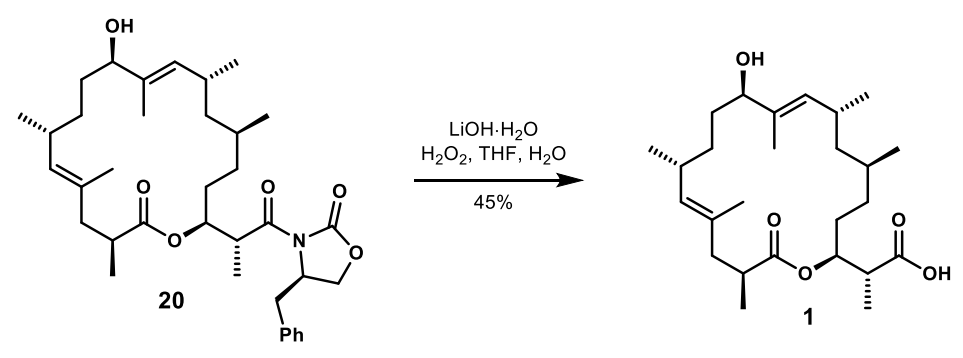

\section{Strasseriolide B (1):}

To a solution of alcohol 20 (2.6 mg, $0.00436 \mathrm{mmol}, 1.0$ equiv) in THF $(0.10 \mathrm{~mL})$ and $\mathrm{H}_{2} \mathrm{O}(0.05$ $\mathrm{mL}$ ) was added $\mathrm{LiOH} \cdot \mathrm{H}_{2} \mathrm{O}\left(1.0 \mathrm{mg}, 0.0175 \mathrm{mmol}, 4.0\right.$ equiv) followed by $30 \%$ aq. $\mathrm{H}_{2} \mathrm{O}_{2}$ solution $(10 \mu \mathrm{L})$. The solution was stirred for $1 \mathrm{~h}$ and then quenched with $3 \mathrm{M} \mathrm{HCl}$. The solution was extracted with EtOAc $(3 \times 3 \mathrm{~mL})$. The combined organic phase was washed with brine, dried with $\mathrm{Na}_{2} \mathrm{SO}_{4}$, filtered, and concentrated in vacuo. The resulting residue was combined with $1.0 \mathrm{mg}$ of crude material from a previous reaction and was purified via prep TLC (50/50/1 EtOAc/hex/AcOH) to obtain strasseriolide B (1.3 mg, 45\%) as a white amorphous solid.

${ }^{1} \mathrm{H}$ NMR $\left(600 \mathrm{MHz}, \mathrm{CD}_{3} \mathrm{OD}\right) \delta 5.17-5.13(\mathrm{~m}, 1 \mathrm{H}), 5.06(\mathrm{~d}, J=9.1 \mathrm{~Hz}, 1 \mathrm{H}), 4.73(\mathrm{~d}, J=8.8$ $\mathrm{Hz}, 1 \mathrm{H}), 3.77(\mathrm{dd}, J=10.2,4.7 \mathrm{~Hz}, 1 \mathrm{H}), 2.75-2.69(\mathrm{~m}, 1 \mathrm{H}), 2.65-2.55(\mathrm{~m}, 2 \mathrm{H}), 2.28-2.20(\mathrm{~m}$, 2H), $2.09(\mathrm{dd}, J=13.7,2.8 \mathrm{~Hz}, 1 \mathrm{H}), 1.79-1.73(\mathrm{~m}, 1 \mathrm{H}), 1.62(\mathrm{~d}, J=0.9 \mathrm{~Hz}, 3 \mathrm{H}), 1.59(\mathrm{~d}, J=1.2$ $\mathrm{Hz}, 3 \mathrm{H}), 1.54-1.48(\mathrm{~m}, 3 \mathrm{H}), 1.44-1.40(\mathrm{~m}, 1 \mathrm{H}), 1.33-1.30(\mathrm{~m}, 3 \mathrm{H}), 1.20(\mathrm{~d}, J=6.9 \mathrm{~Hz}, 4 \mathrm{H})$, $1.13(\mathrm{~d}, J=6.9 \mathrm{~Hz}, 4 \mathrm{H}), 1.08-1.05(\mathrm{~m}, 1 \mathrm{H}), 0.97(\mathrm{~d}, J=6.7 \mathrm{~Hz}, 3 \mathrm{H}), 0.92-0.90(\mathrm{~m}, 1 \mathrm{H}), 0.89$ (d, $J=6.7 \mathrm{~Hz}, 3 \mathrm{H}), 0.85(\mathrm{~d}, J=6.7 \mathrm{~Hz}, 3 \mathrm{H})$.

${ }^{13} \mathrm{C}\left\{{ }^{1} \mathrm{H}\right\}$ NMR $\left(151 \mathrm{MHz}, \mathrm{CD}_{3} \mathrm{OD}\right) \delta$ 177.6, 176.6, 136.9, 135.9, 134.9, 133.7, 80.4, 75.5, 48.2, 45.2, 42.9, 42.5, 35.0, 34.1, 33.3, 31.8, 30.8, 30.7, 30.4, 22.4, 22.3, 21.8, 18.9, 17.2, 14.1, 10.6.

HRMS (ESI-TOF) m/z: Calcd for $\mathrm{C}_{26} \mathrm{H}_{42} \mathrm{O}_{5} \mathrm{Na}^{+}[\mathrm{M}+\mathrm{Na}]^{+}$459.3087; Found 459.3078 .

$[\alpha]^{22} \mathrm{D}=+20.0\left(c=0.25, \mathrm{CH}_{3} \mathrm{OH}\right)$. Literature reported: $[\alpha]^{25} \mathrm{D}=+32.6\left(c=0.25, \mathrm{CH}_{3} \mathrm{OH}\right) .{ }^{12}$ 


\section{References}

1. Comeau, C.; Reis, B.; Stadelmann, T.; Tremblay, J.; Poulet, S.; Fröhlich, U.; Côté, J.; Boudreault, P.; Derbali, R. M.; Sarret, P.; Grandbois, M.; Leclair, G.; Riniker, S.; Marsault, É. Modulation of the Passive Permeability of Semipeptidic Macrocycles: N- and C-Methylations Fine-Tune Conformation and Properties. J. Med. Chem. 2021, 64, 5365-5383.

2. Lu, H.; Martinez, M. D.; Shenvi, R. A. An eight-step gram-scale synthesis of (-)-jiadifenolide. Nat. Chem. 2015, 7, 604-607.

3. Schultz, E. E.; Sarpong, R. Application of In Situ-Generated Rh-Bound Trimethylenemethane Variants to the Synthesis of 3,4-Fused Pyrroles. J. Am. Chem. Soc. 2013, 135, 4696-4699.

4. Buchwald, S. L.; LaMaire, S. J.; Nielsen, R. B.; Watson, B. T.; King, S. M. Schwartz Reagent. Org. Synth. 1993, 71, 77.

5. Featherston, A. L.; Miller, S. L. Synthesis and evaluation of phenylalanine-derived trifluoromethyl ketones for peptide-based oxidation catalysis. Bioorg. Med. Chem. 2016, 24 , 4871-4874.

6. Nicolaou, K. C.; Estrada, A. A.; Zak, M.; Lee, S. H.; Safina, B. S. A Mild and Selective Method for the Hydrolysis of Esters with Trimethyltin Hydroxide. Angew. Chem. Int. Ed. 2005, 44, 1378-1382.

7. Keck, G. E.; Boden, E. P.; Mabury, S. A. A useful Wittig Reagent for the Stereoselective Synthesis of Trans $\alpha, \beta$-Unsaturated Thiol Esters. J. Org. Chem. 1985, 50, 709-710.

8. Des Mazery, R.; Pullez, M.; López, F.; Harutyunyan, S. R.; Minnaard, A. J.; Feringa, B. L. An Iterative Catalytic Route to Enantiopure Deoxypropionate Subunits: Asymmetric Conjugate Addition of Grignard Reagents to $\alpha, \beta$-Unsaturated Thioesters. J. Am. Chem. Soc. 2005, 127, 9966-9967.

9. Hess, S. N.; Mo, X.; Wirtz, C.; Fürstner, A. Total Synthesis of Limaol. J. Am. Chem. Soc. 2021, 143, 2464-2469.

10. Evans, D. A.; Kaldor, S. W.; Jones, T. K.; Clardy, J.; Stout, T. J. Total synthesis of the macrolide antibiotic cytovaricin. J. Am. Chem. Soc. 1990, 112, 7001-7031.

11. (a) Inoue, T.; Mukaiyama, T. Regio- and Stereoselective Cross-aldol Reactions via Dialkylboryl Triflates. Bull. Chem. Soc. Jpn. 1980, 53, 174-178. (b) Evans, D. A.; Nelson, J. V.; Vogel, E.; Taber, T. R. Stereoselective Aldol Condensations via Boron Enolates. J. Am. Chem. Soc. 1981, 103, 3099-3111.

12. Annang, F.; Pérez-Moreno, G.; González-Menéndez, V.; Lacret, R.; Pérez-Victoria, I.; Martín, J.; Cantizani, J.; Pedro, N. de; Choquesillo-Lazarte, D.; Ruiz-Pérez, L. M.; GonzálezPacanowska, D.; Genilloud, O.; Vicente, F.; Reyes, F. Strasseriolides A-D, A Family of Antiplasmodial Macrolides Isolated from the Fungus Strasseria Geniculata CF-247251. Org. Lett. 2020, 22, 6709-6713. 


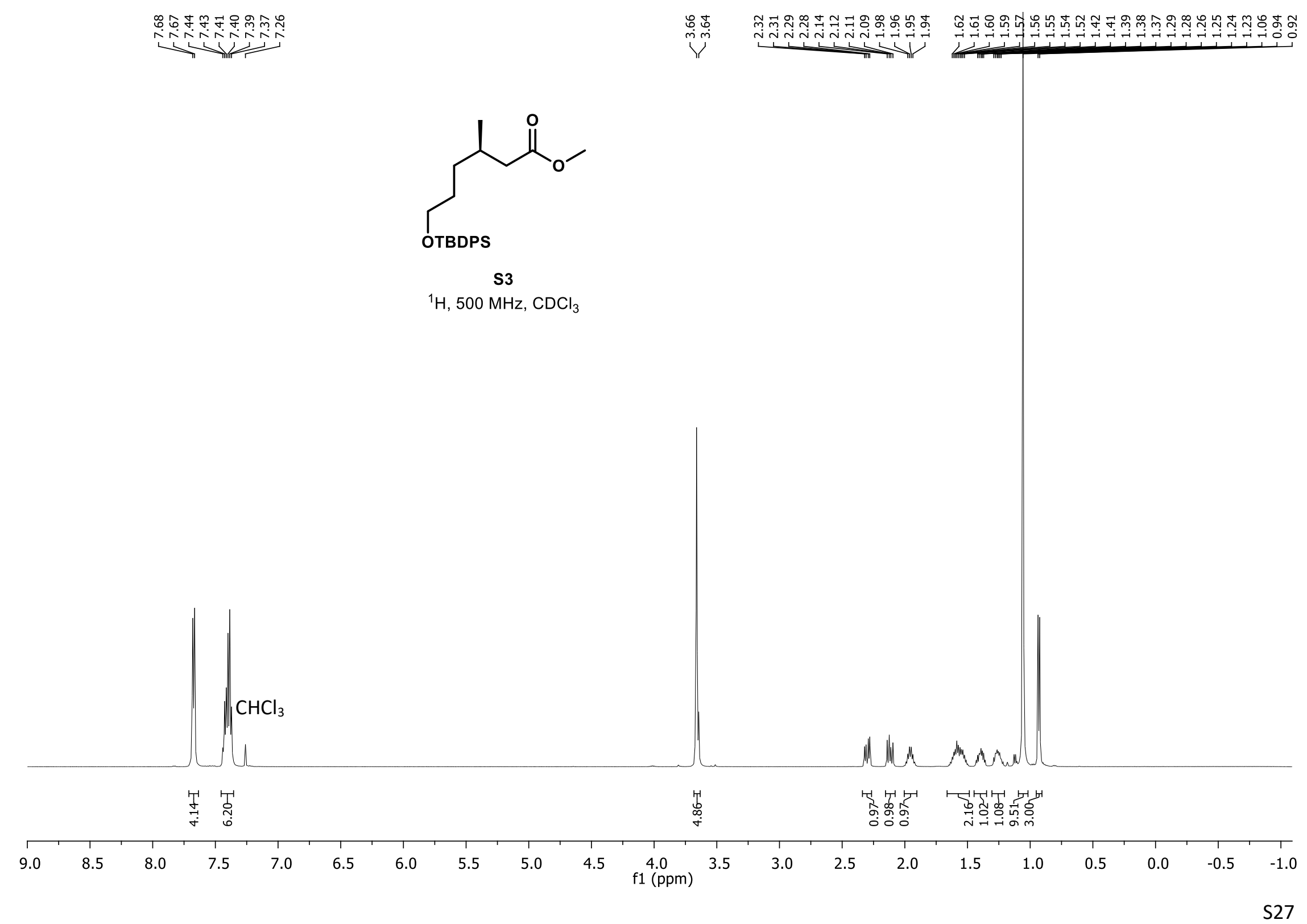




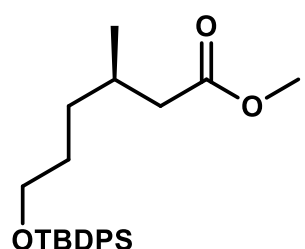

S3

${ }^{13} \mathrm{C}, 126 \mathrm{MHz}, \mathrm{CDCl}_{3}$

倸

$\mathrm{CDCl}_{3}$

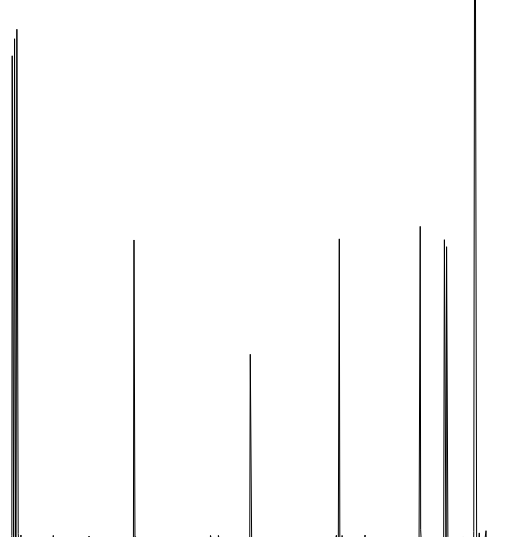

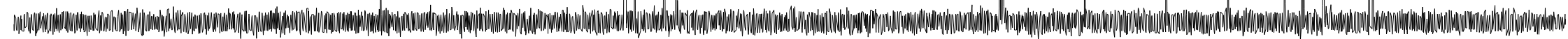

230

$220 \quad 210 \quad 200 \quad 190 \quad 180$

170

160

140

130

$120 \begin{aligned} & 110 \\ & \mathrm{f} 1(\mathrm{ppm})\end{aligned}$

100

90

80

60

50

40

30

20

10 


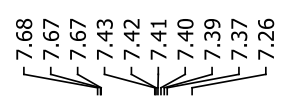

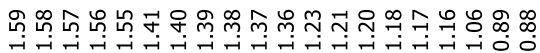

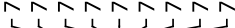

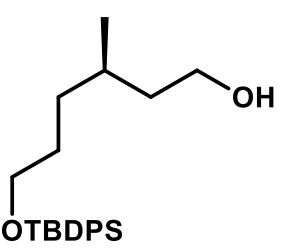

S4

${ }^{1} \mathrm{H}, 500 \mathrm{MHz}, \mathrm{CDCl}_{3}$

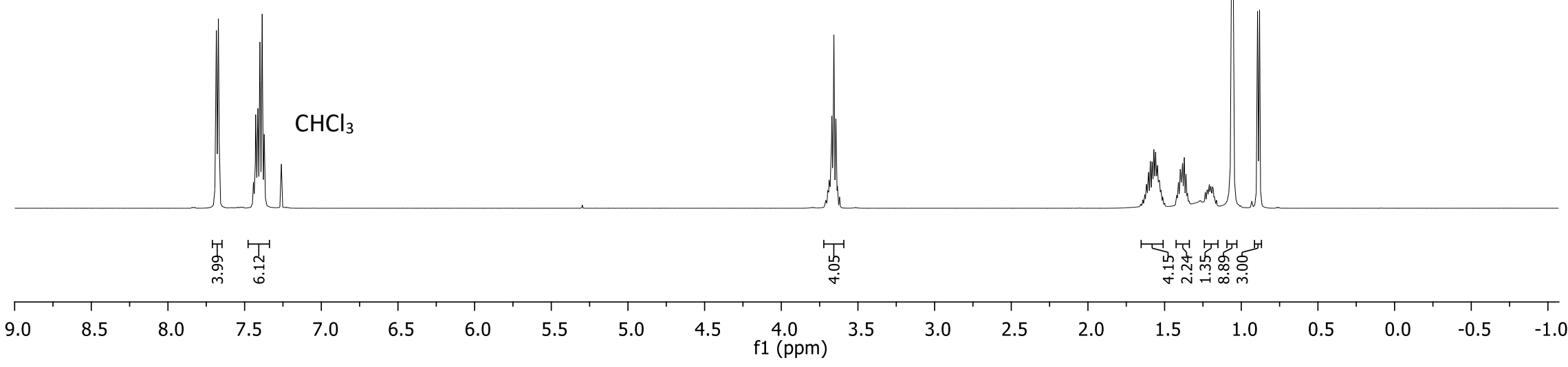



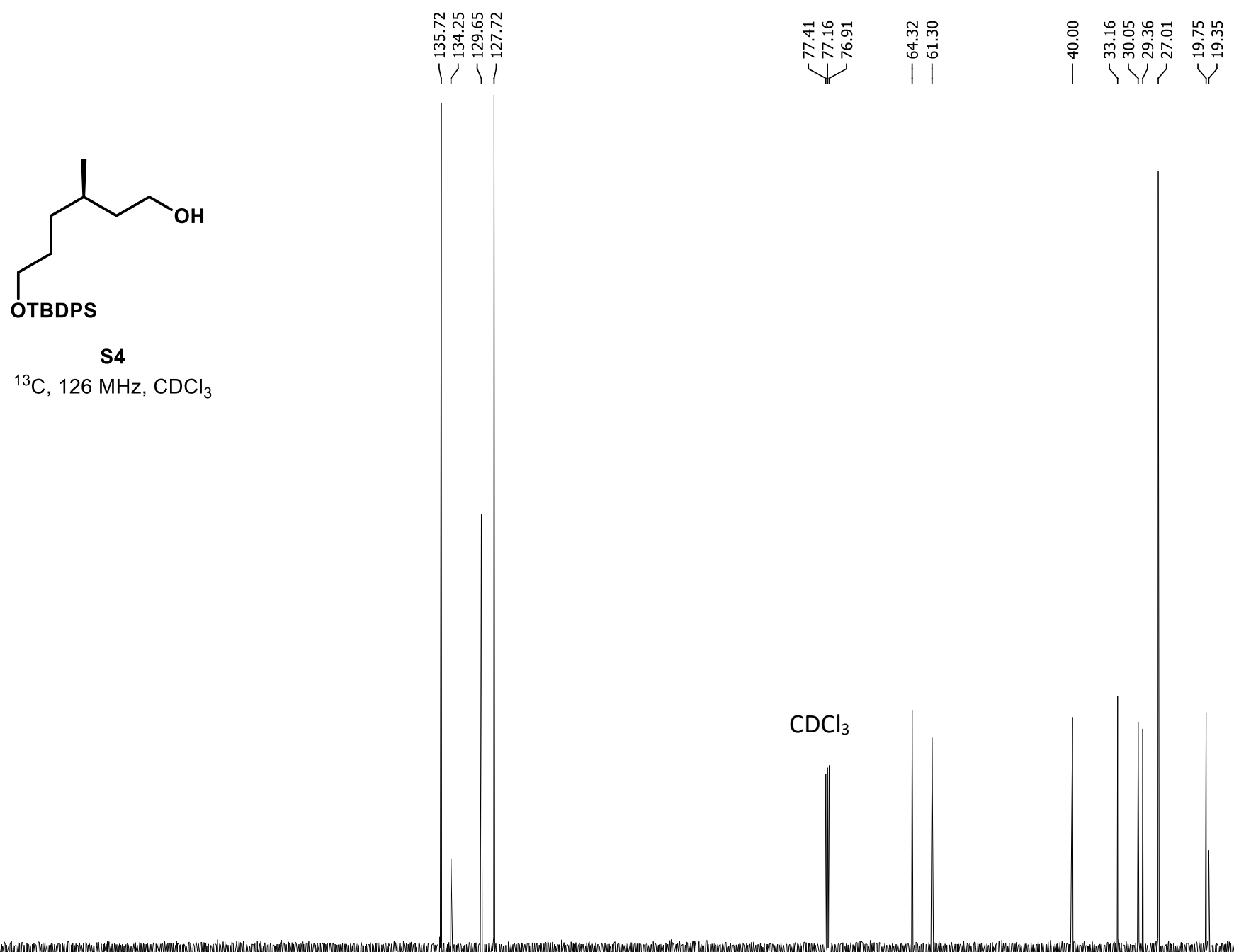

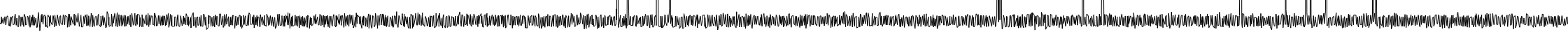

${ }^{13} \mathrm{C}, 126 \mathrm{MHz}, \mathrm{CDCl}_{3}$

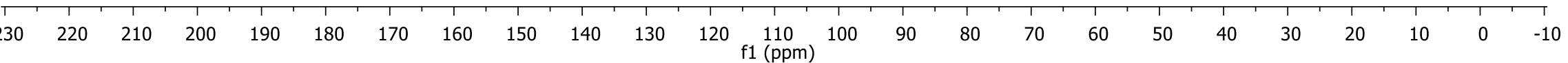




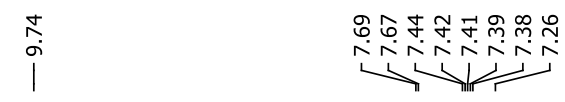

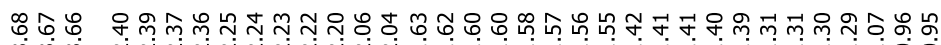

nNnNnNnNa

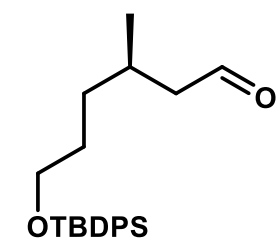

${ }^{1} \mathrm{H}, 500 \mathrm{MHz}, \mathrm{CDCl}_{3}$

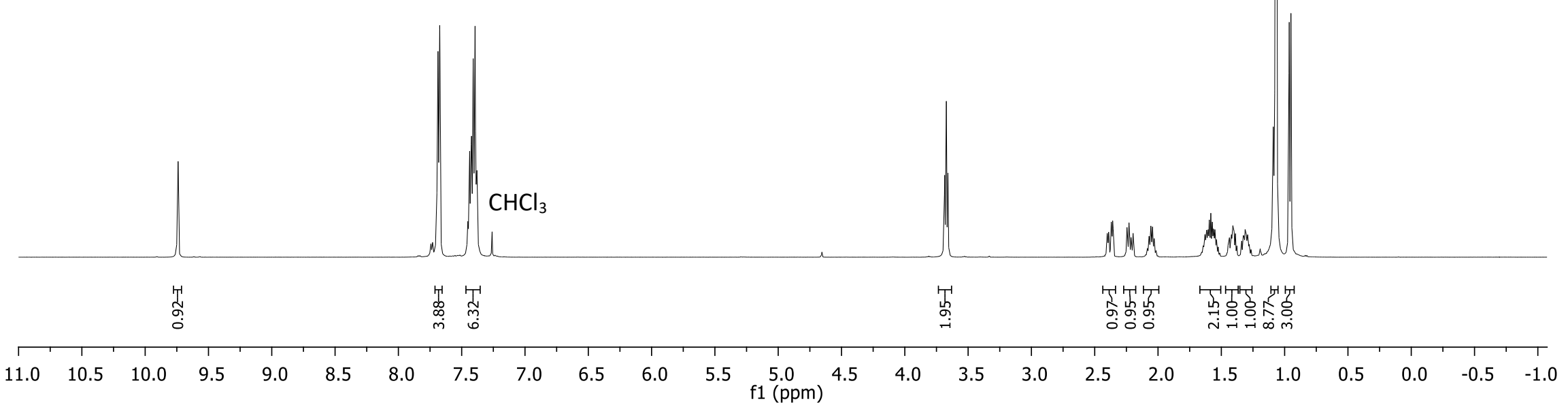



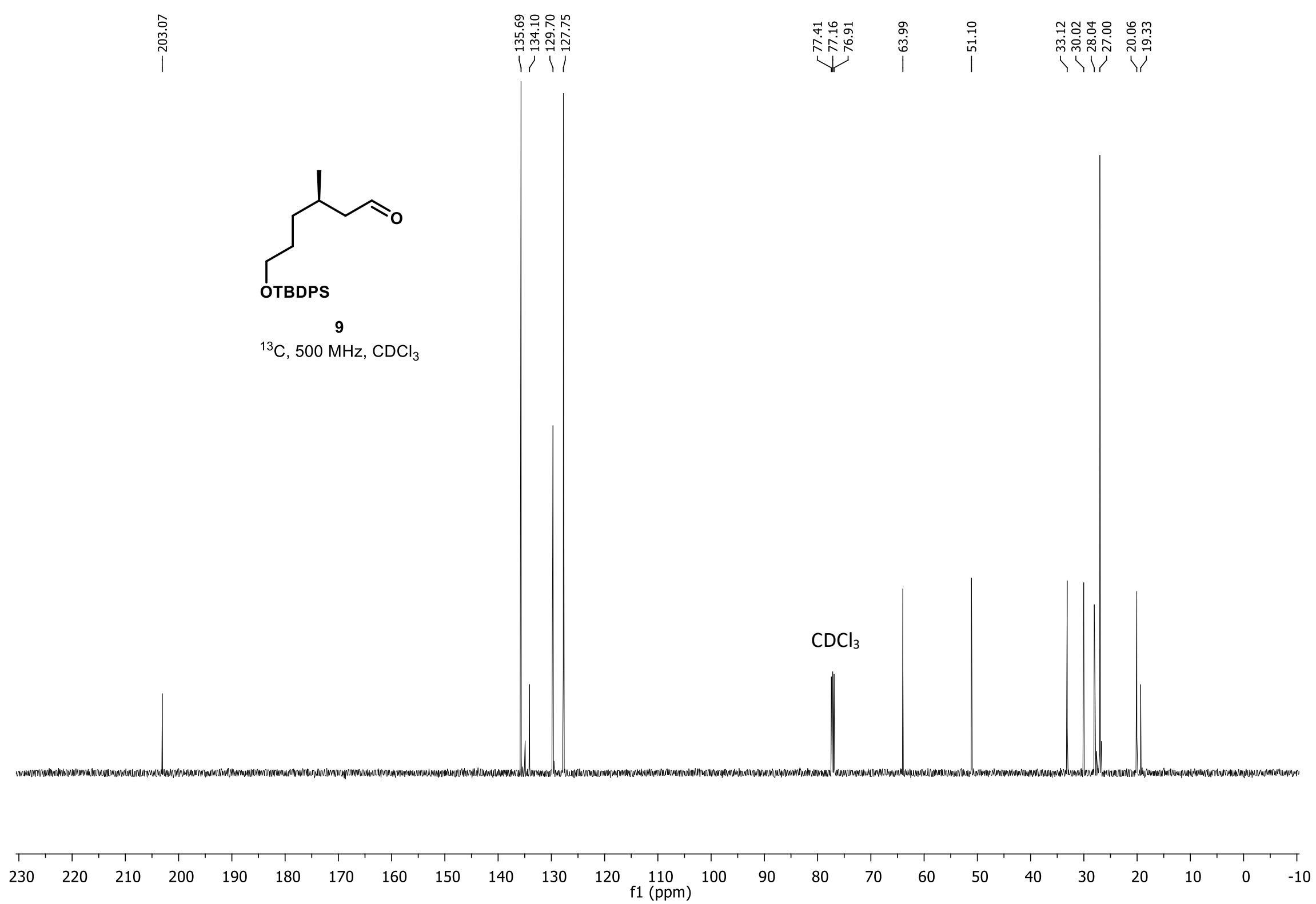

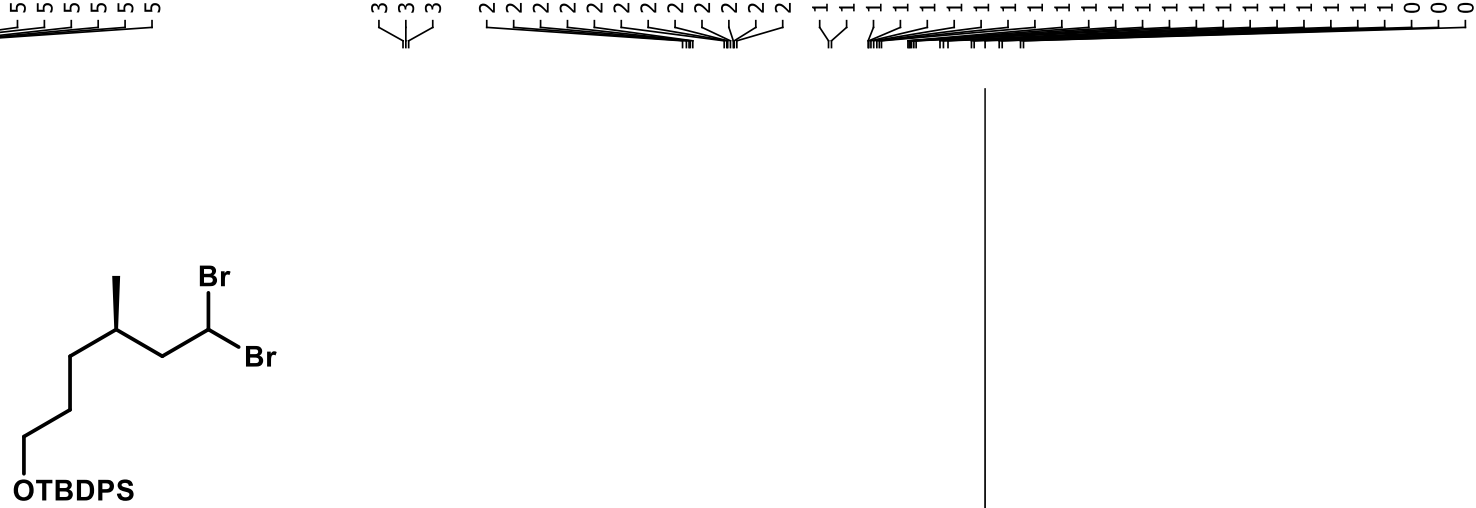

S5

${ }^{1} \mathrm{H}, 500 \mathrm{MHz}, \mathrm{CDCl}_{3}$
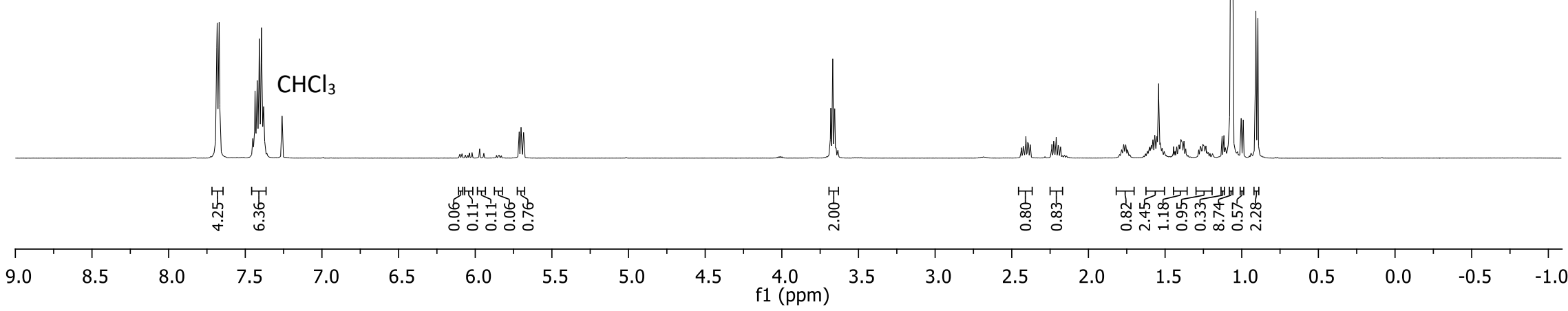


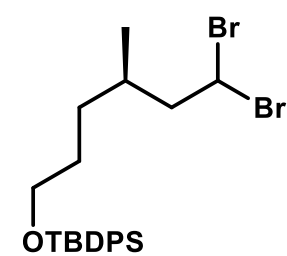

s5

${ }^{13} \mathrm{C}, 126 \mathrm{MHz}, \mathrm{CDCl}_{3}$

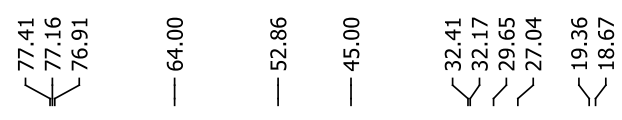

$\mathrm{CDCl}_{3}$

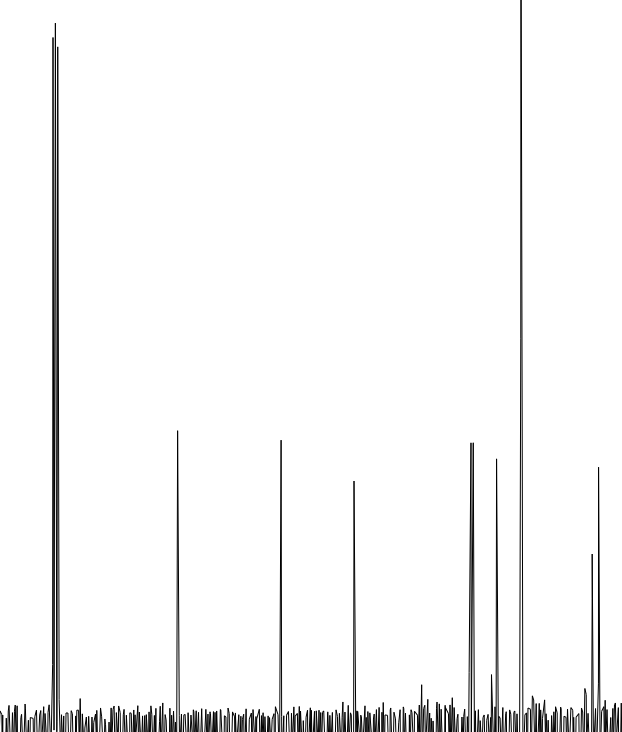

$\begin{array}{lllllllllllllllllllllllll}230 & 220 & 210 & 200 & 190 & 180 & 170 & 160 & 150 & 140 & 130 & 120 & \begin{array}{l}110 \\ \mathrm{f} 1(\mathrm{ppm})\end{array} & 100 & 90 & 80 & 70 & 60 & 50 & 40 & 30 & 20 & 10 & 0 & -10\end{array}$ 


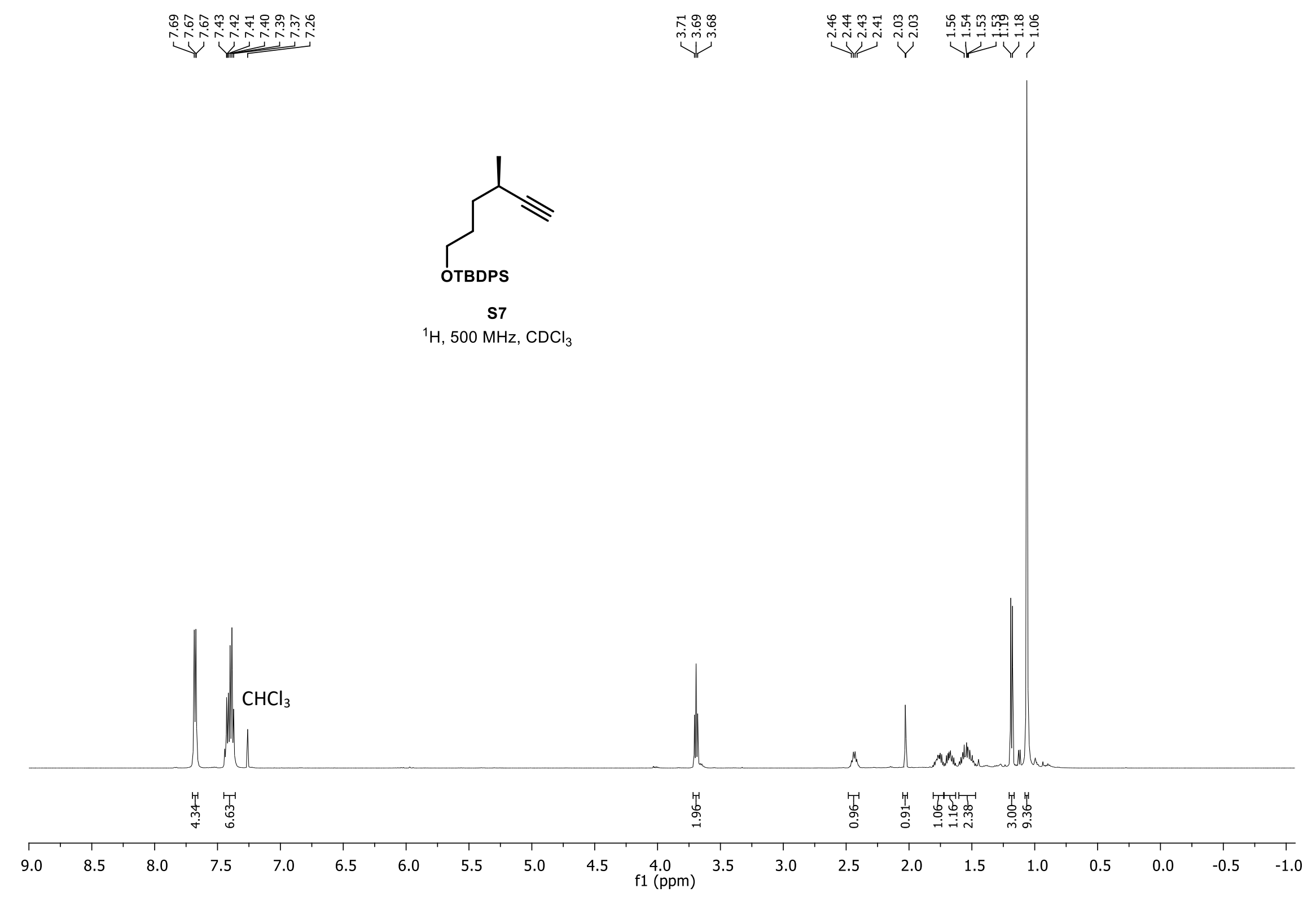




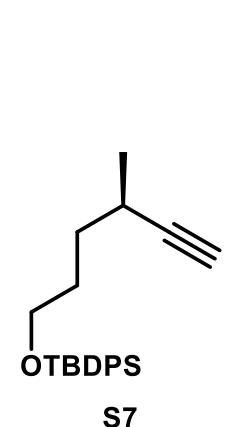

S7

${ }^{13} \mathrm{C}, 126 \mathrm{MHz}, \mathrm{CDCl}_{3}$

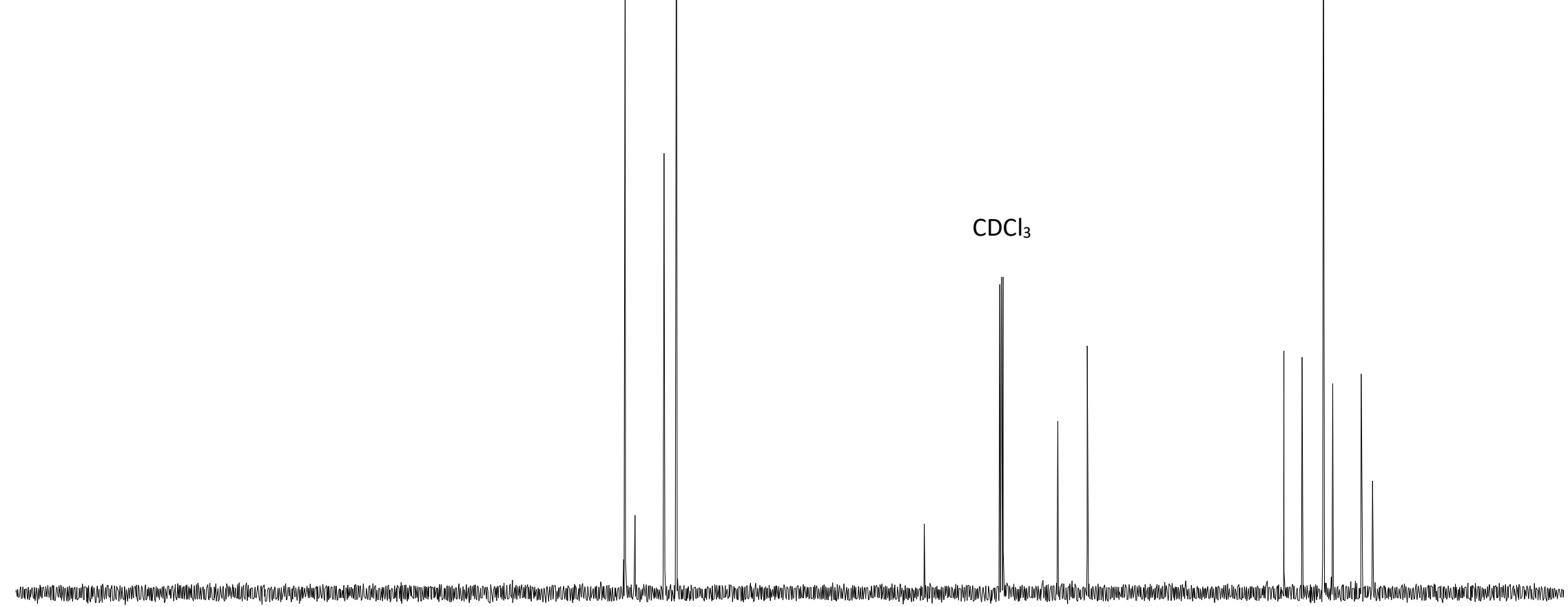

$\begin{array}{lllllllllllllllllllllllll}230 & 220 & 210 & 200 & 190 & 180 & 170 & 160 & 150 & 140 & 130 & 120 & \begin{array}{l}110 \\ \mathrm{f} 1(\mathrm{ppm})\end{array} & 100 & 90 & 80 & 70 & 60 & 50 & 40 & 30 & 20 & 10 & 0 & -10\end{array}$ 


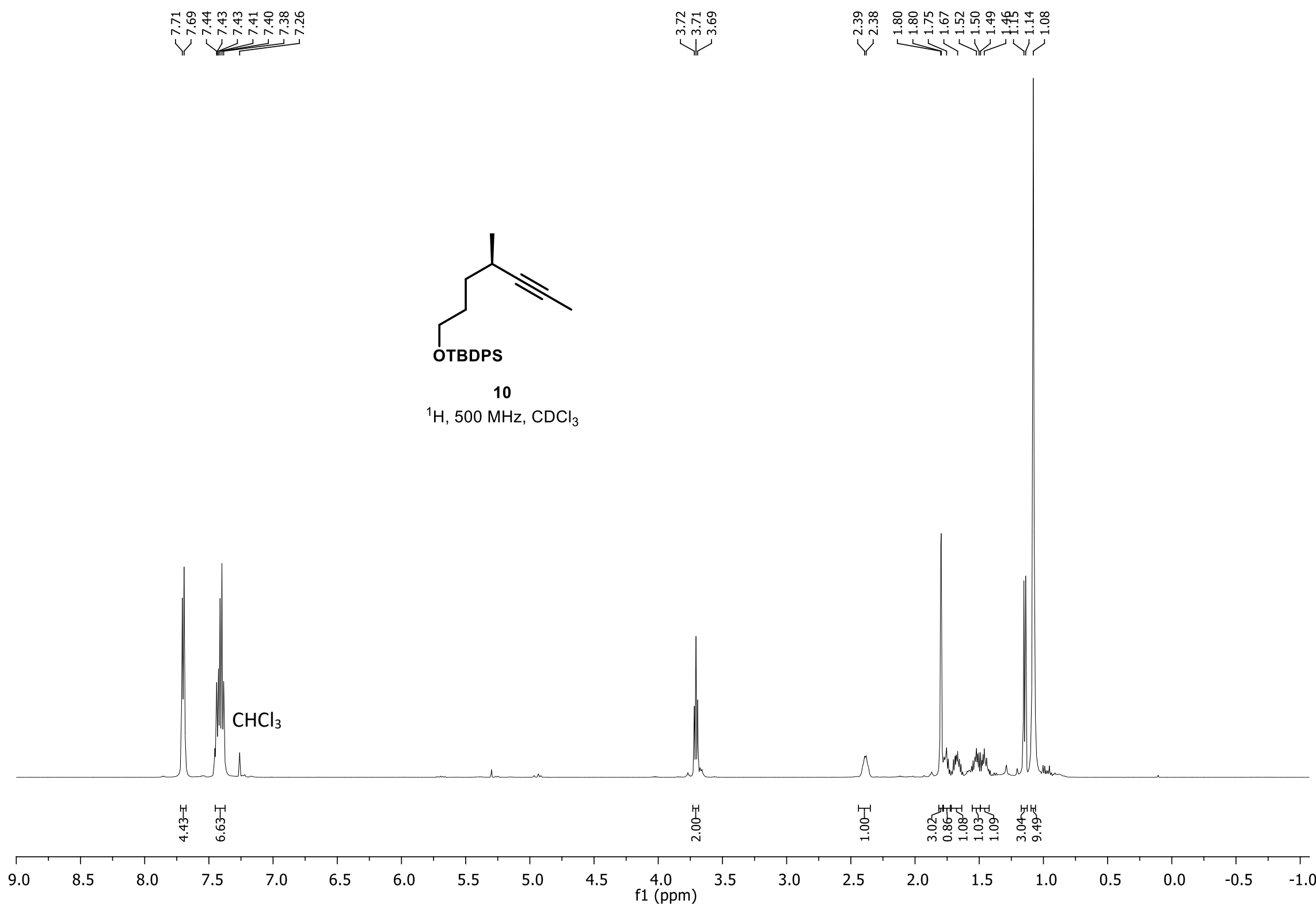

ஸึ่ 


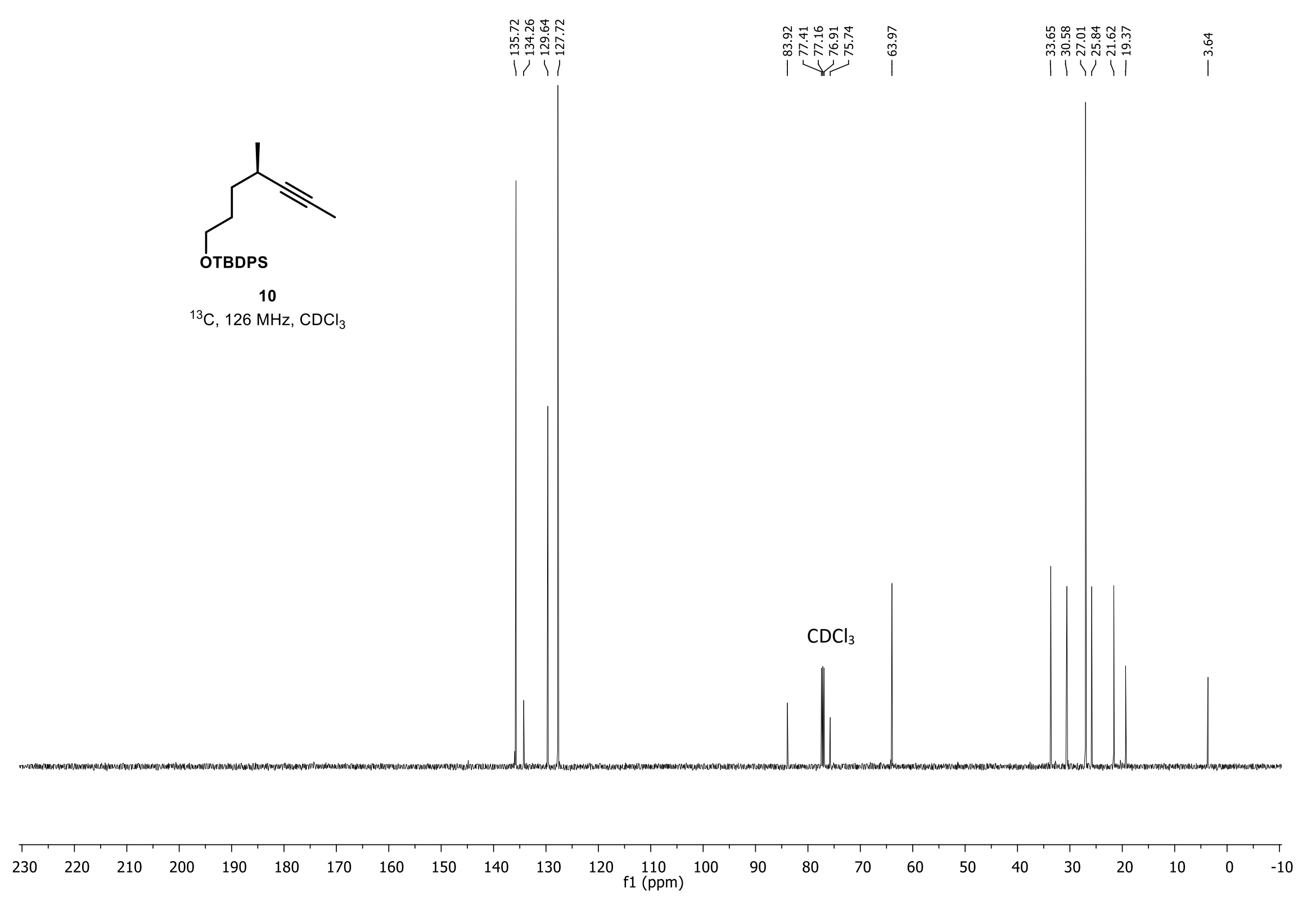




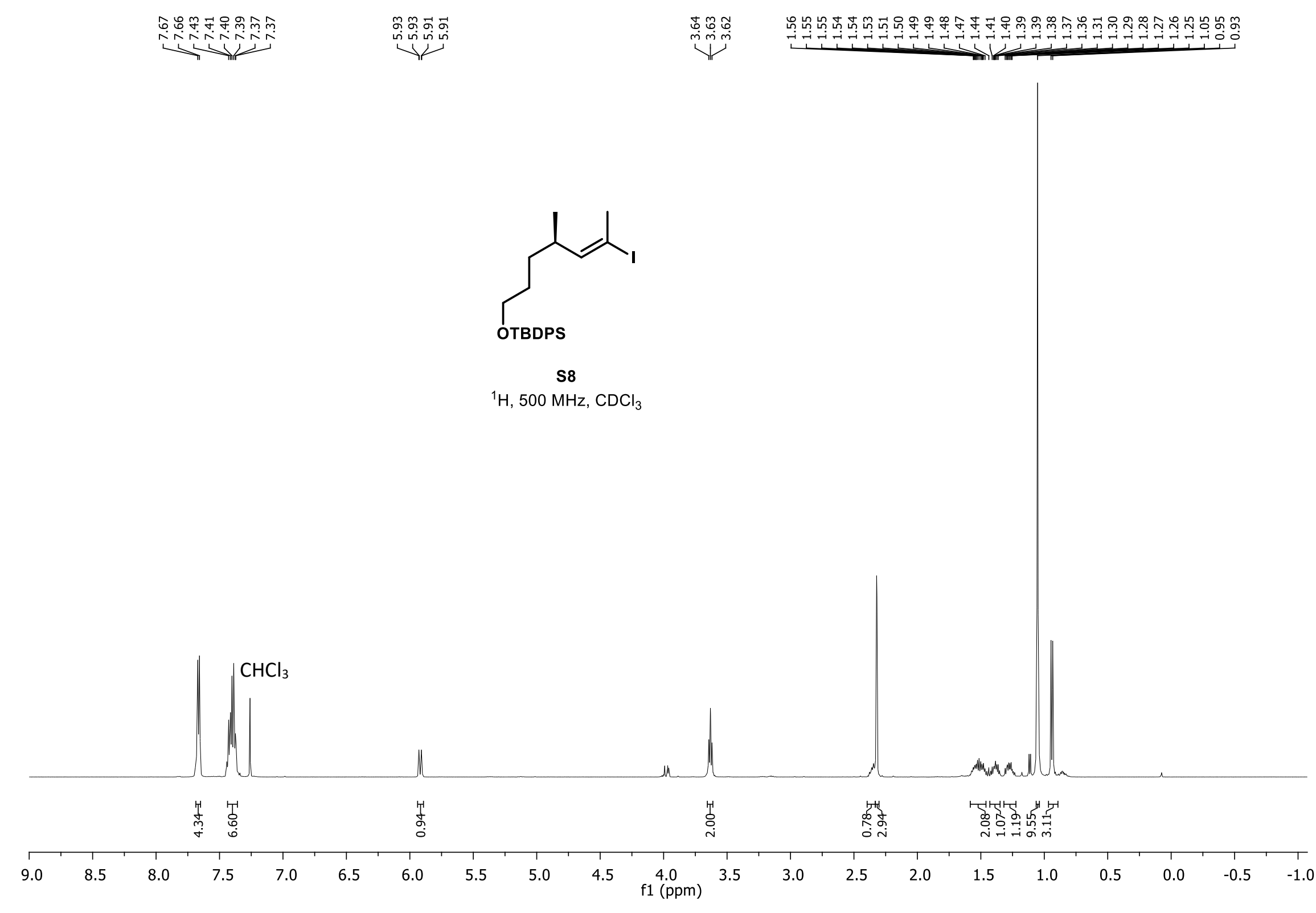




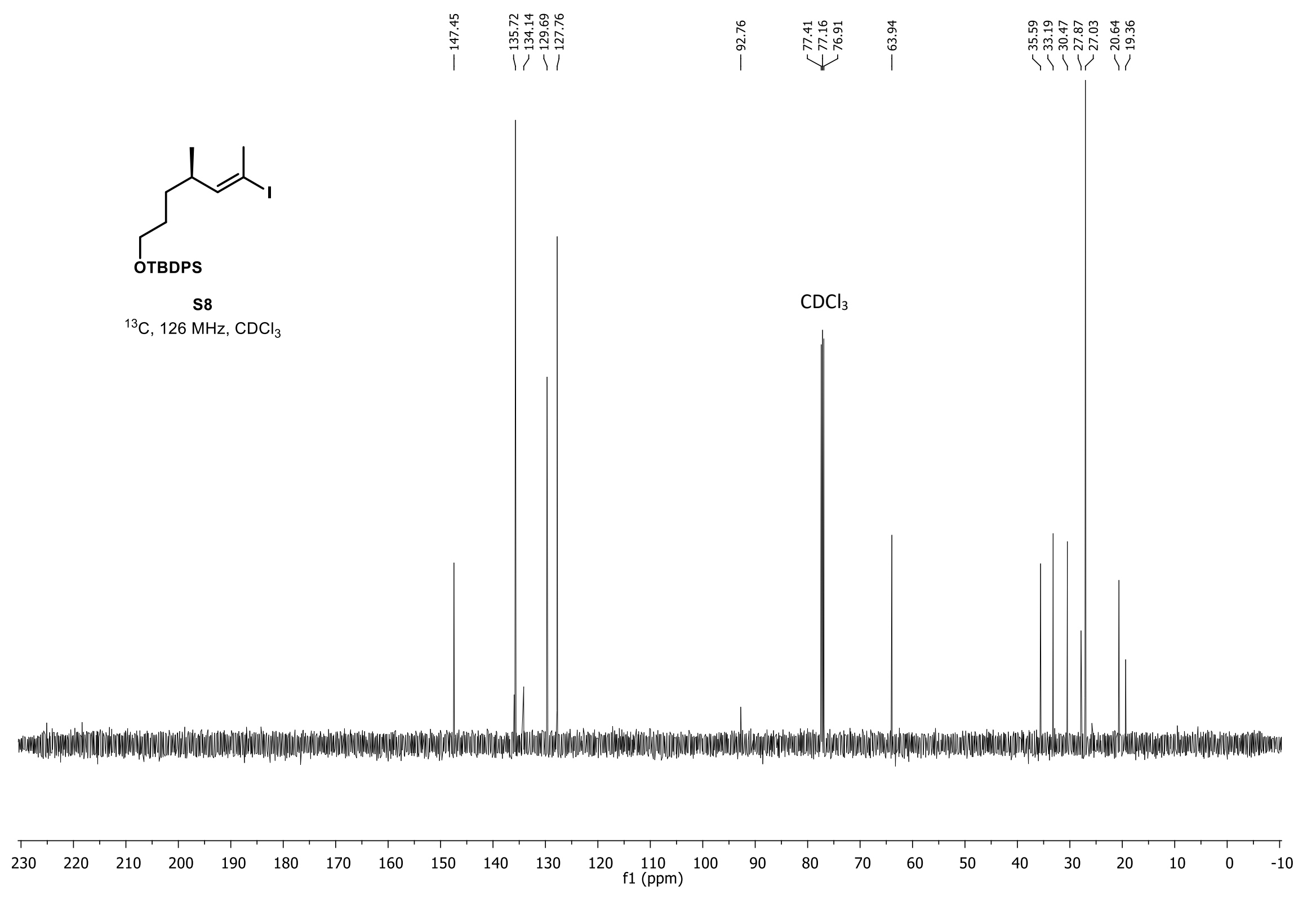




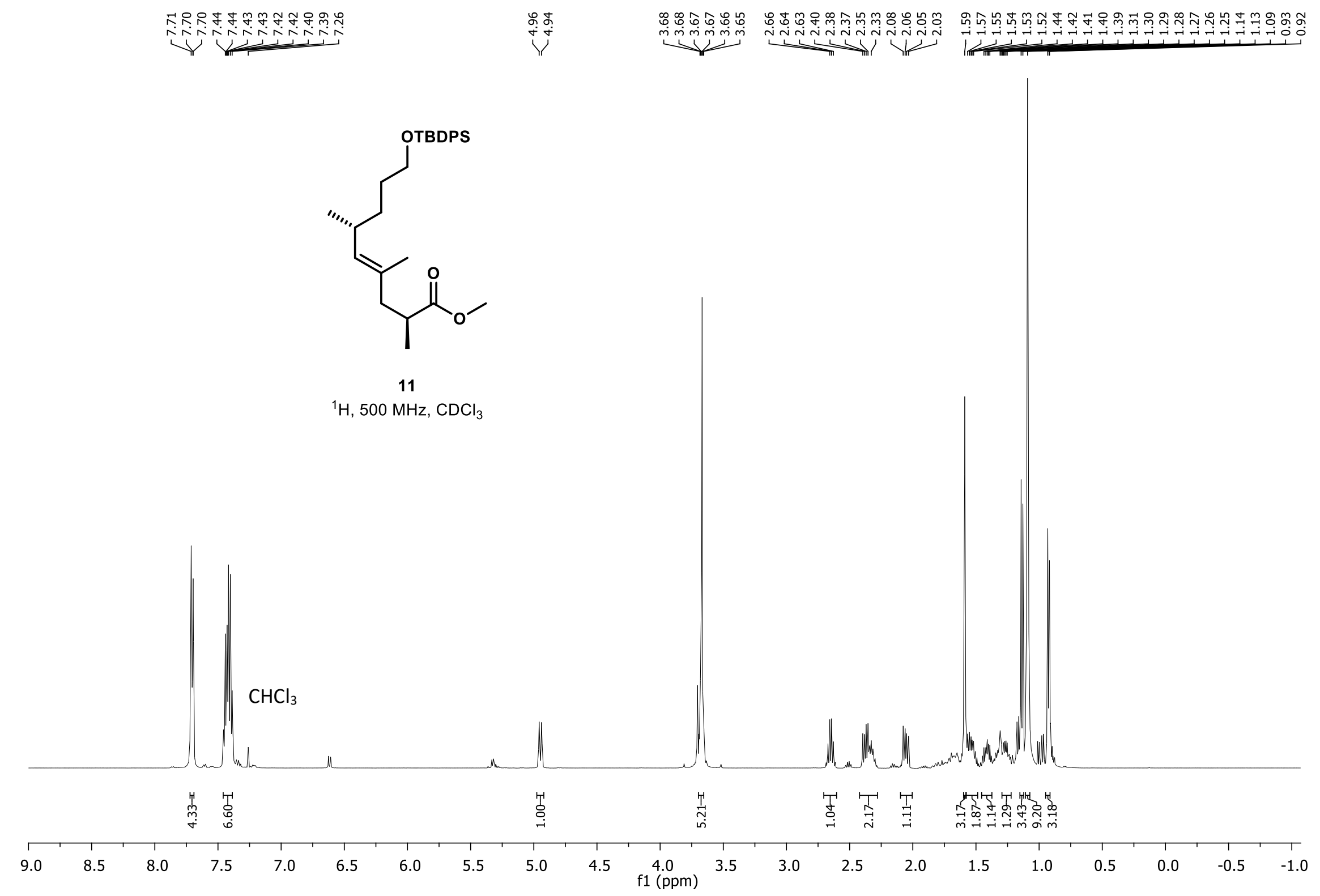



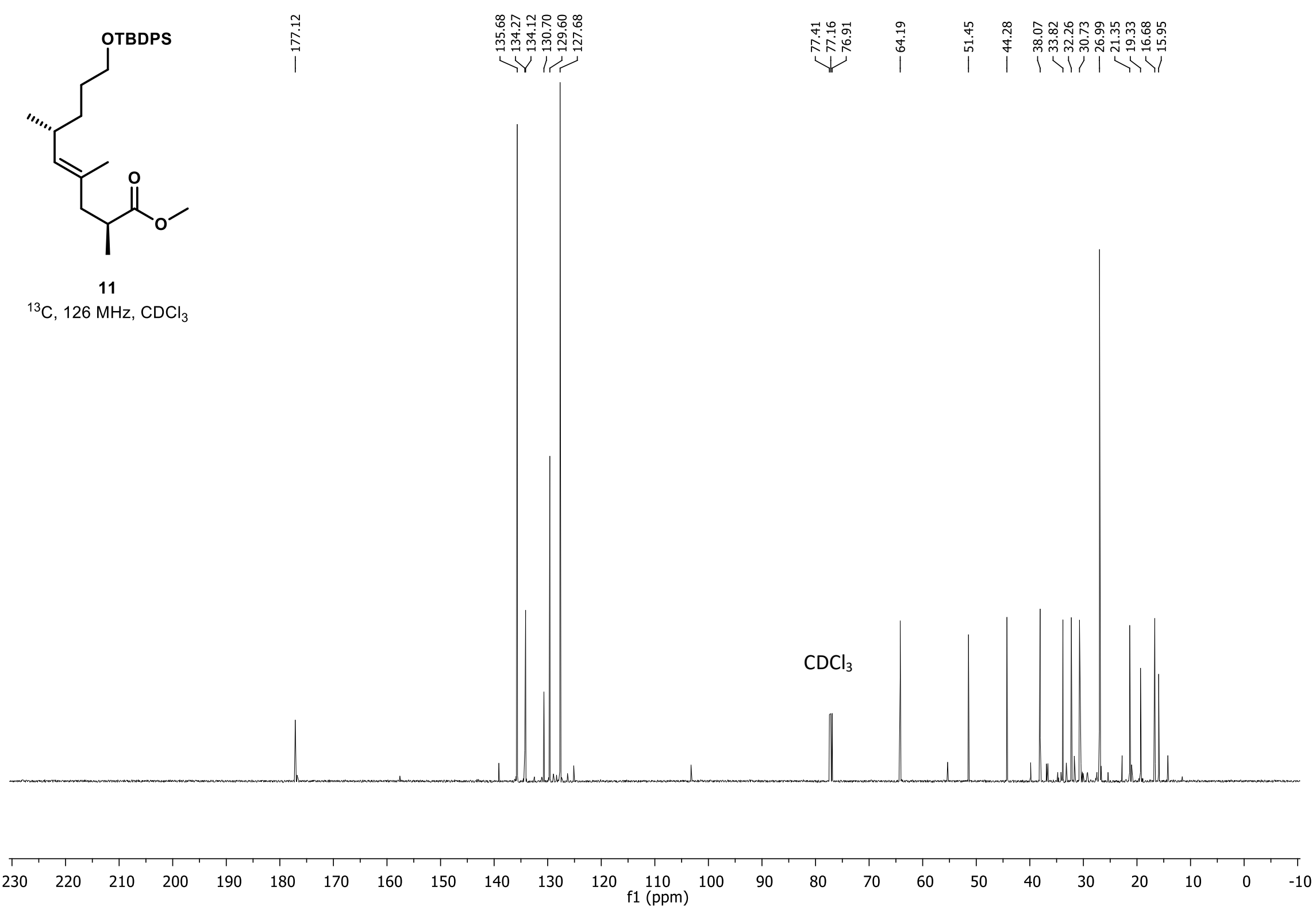


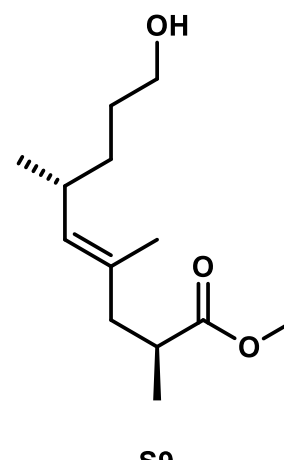

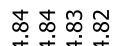

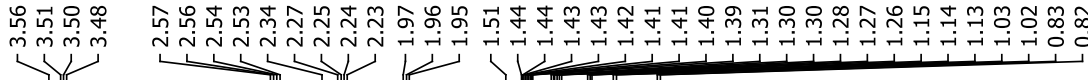

${ }^{1} \mathrm{H}, 500 \mathrm{MHz}, \mathrm{CDCl}_{3}$

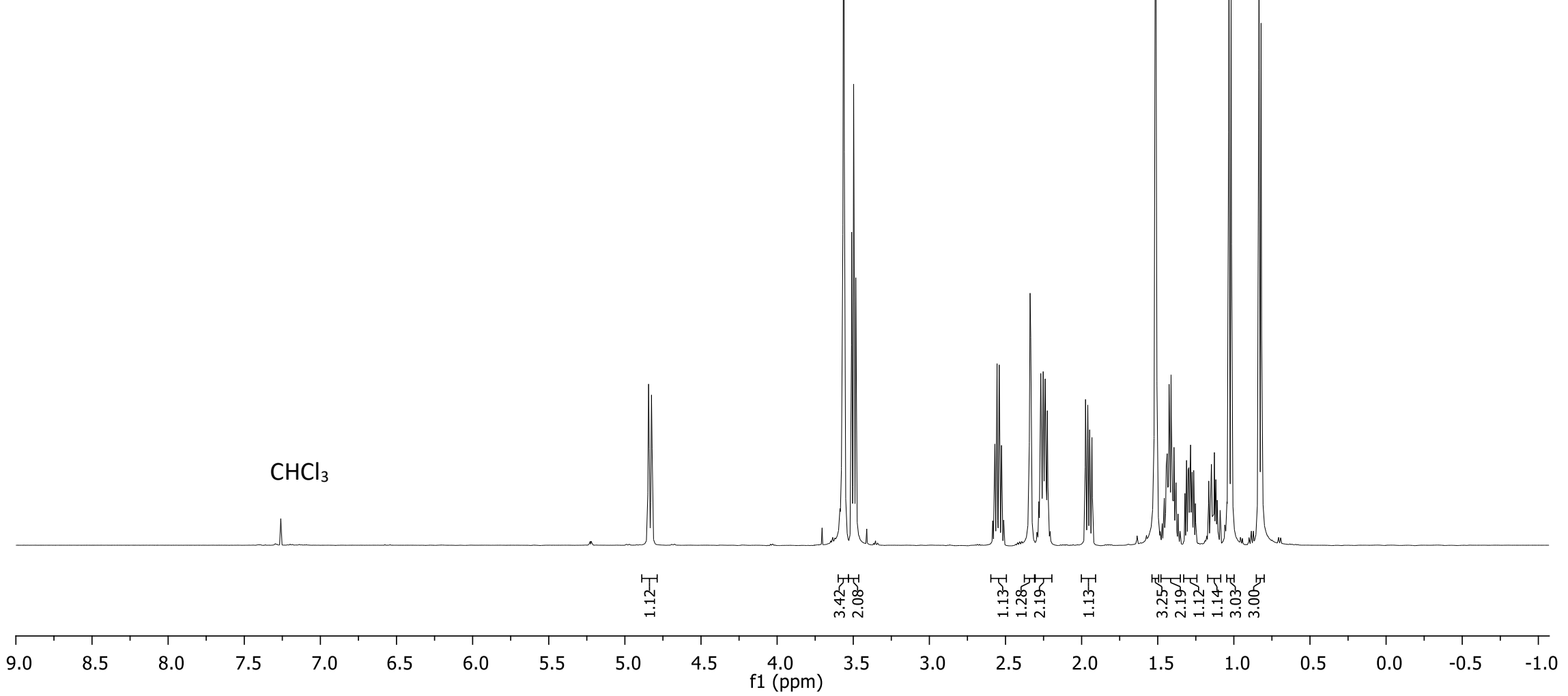




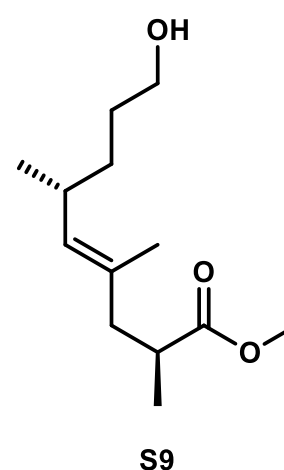

年

年

${ }^{13} \mathrm{C}, 126 \mathrm{MHz}, \mathrm{CDCl}_{3}$

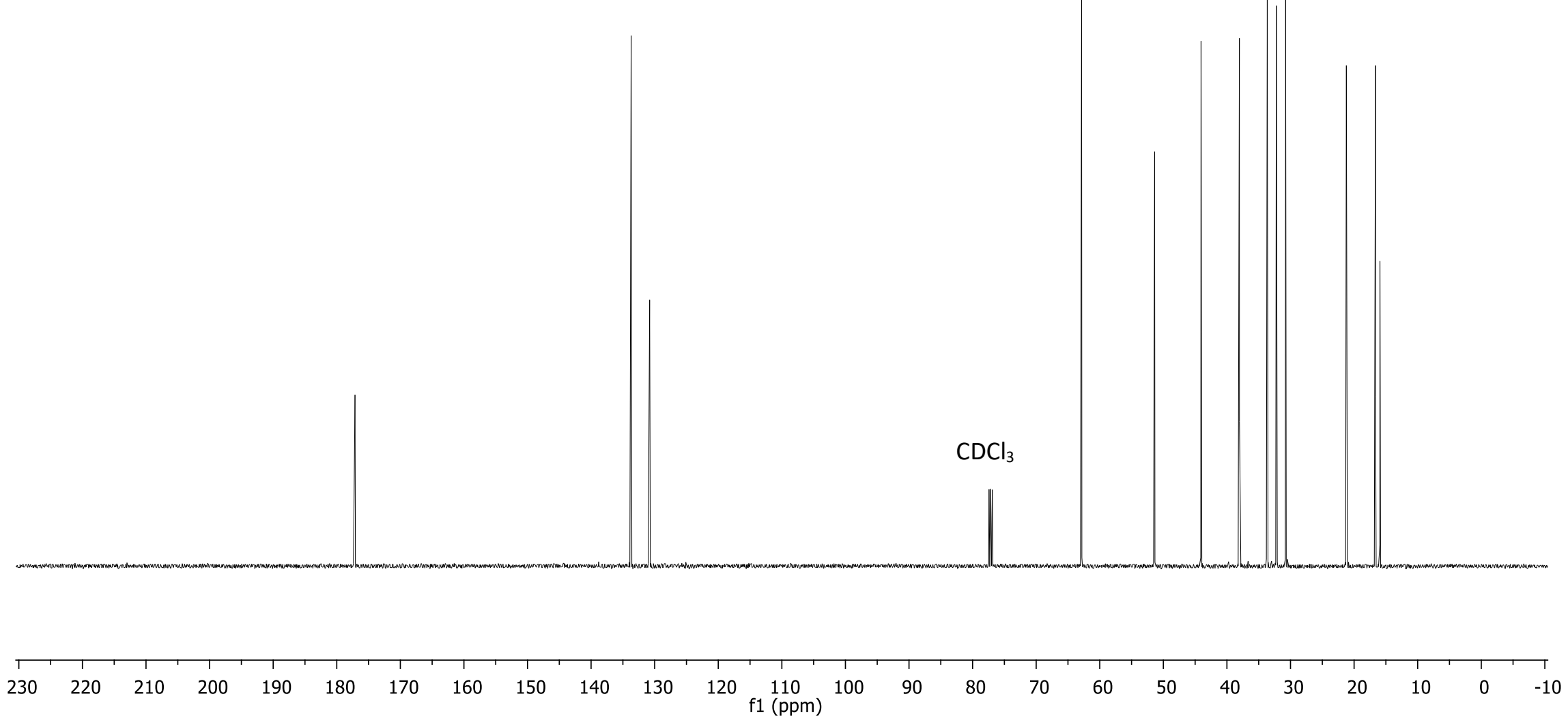




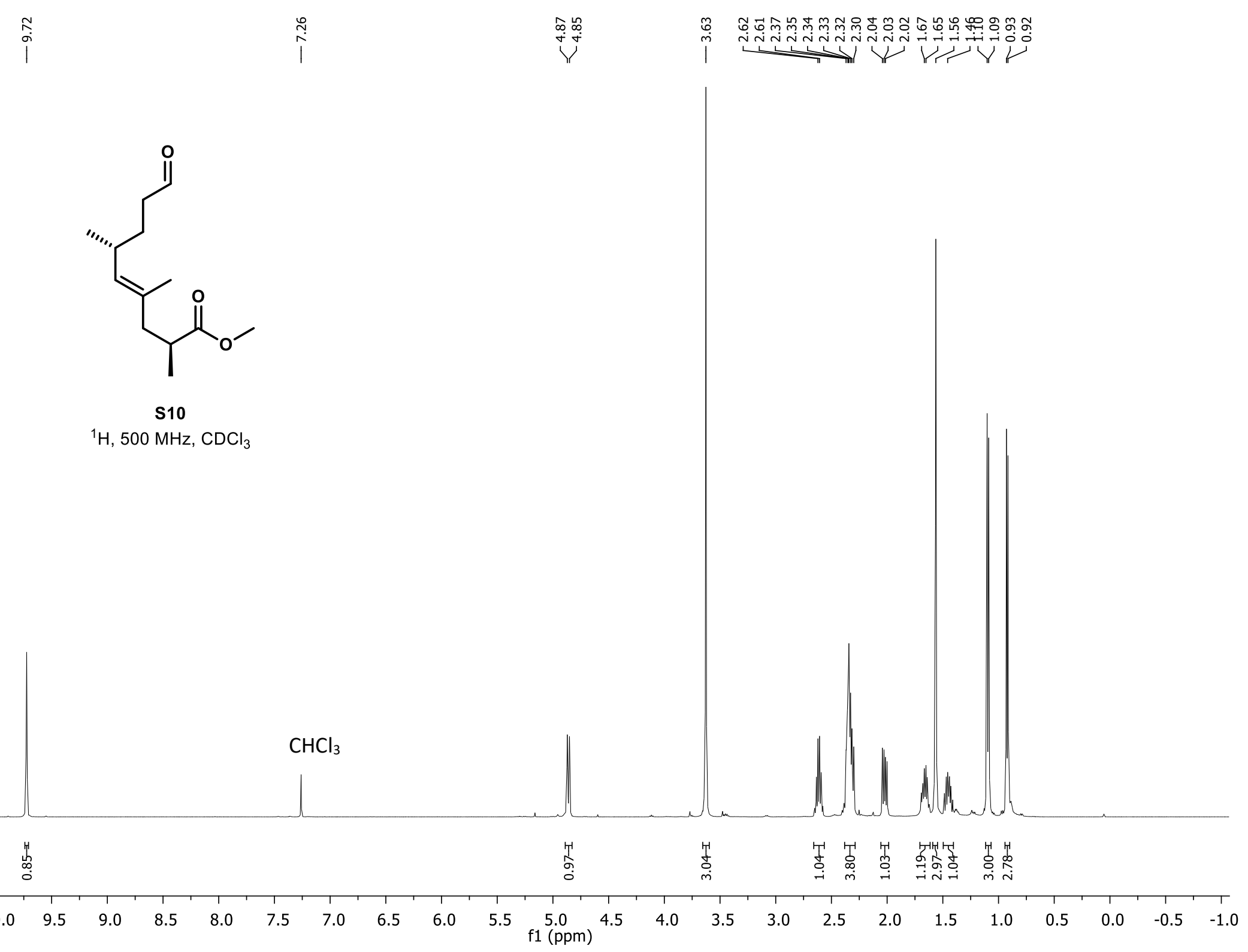



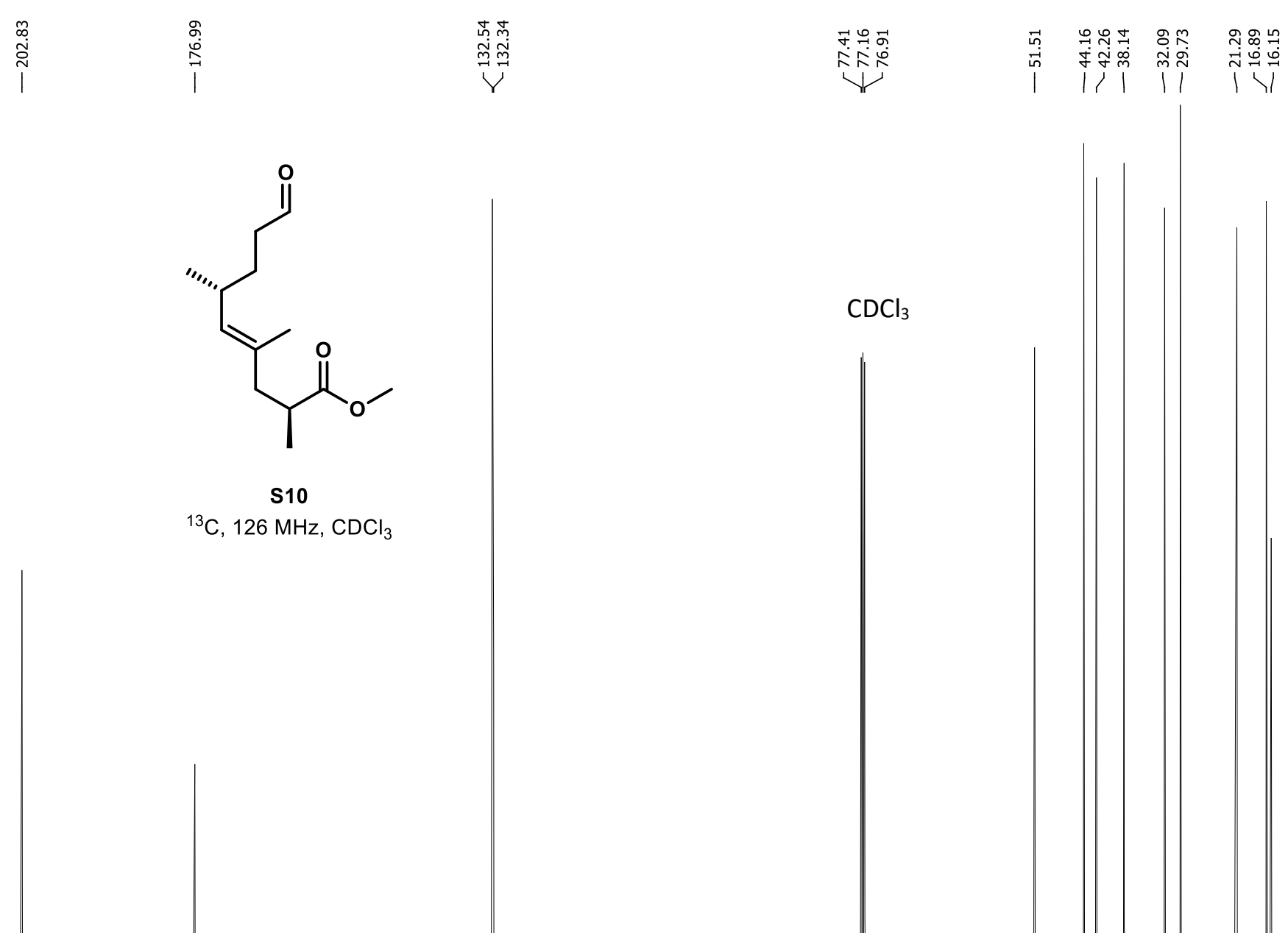

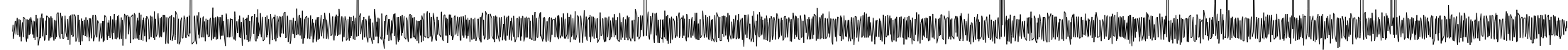

${ }^{13} \mathrm{C}, 126 \mathrm{MHz}, \mathrm{CDCl}_{3}$

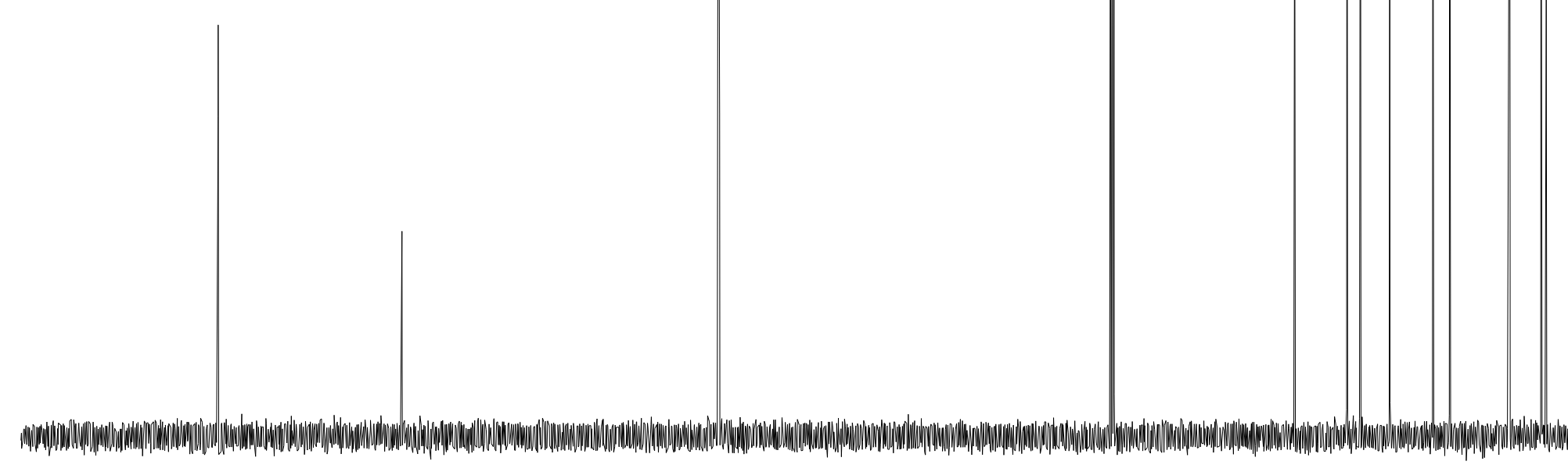




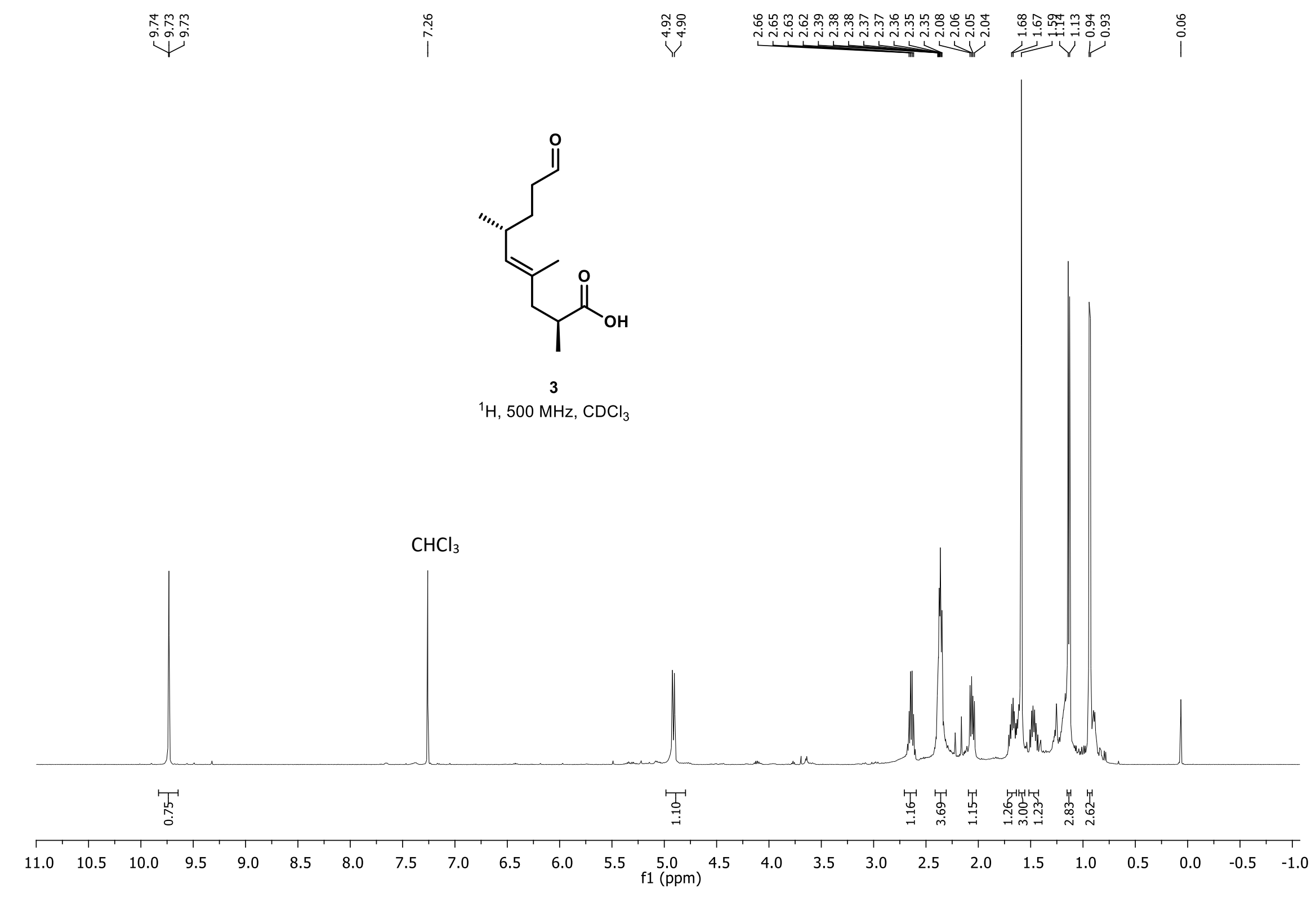




I

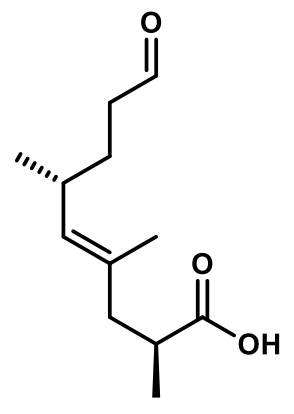

3

${ }^{13} \mathrm{C}, 126 \mathrm{MHz}, \mathrm{CDCl}_{3}$

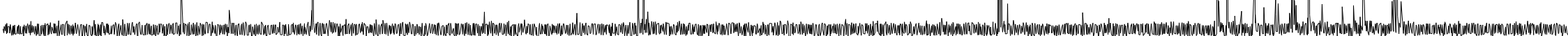

$\mathrm{CDCl}_{3}$
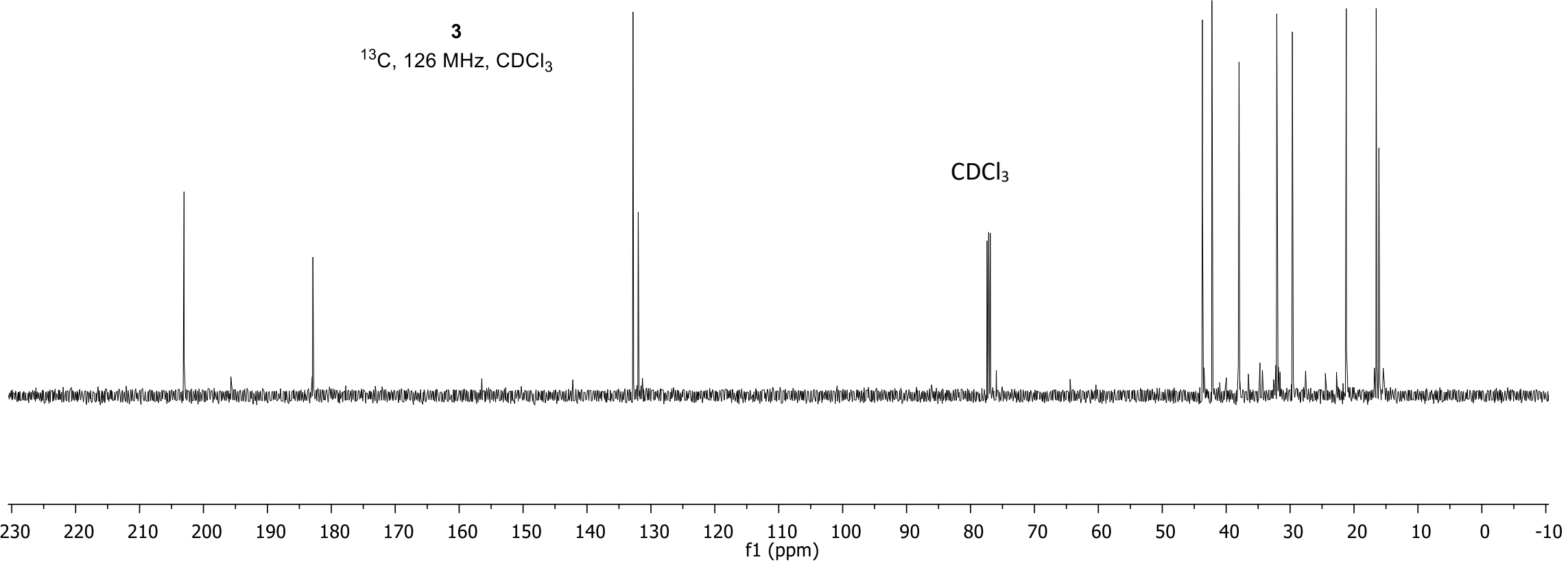


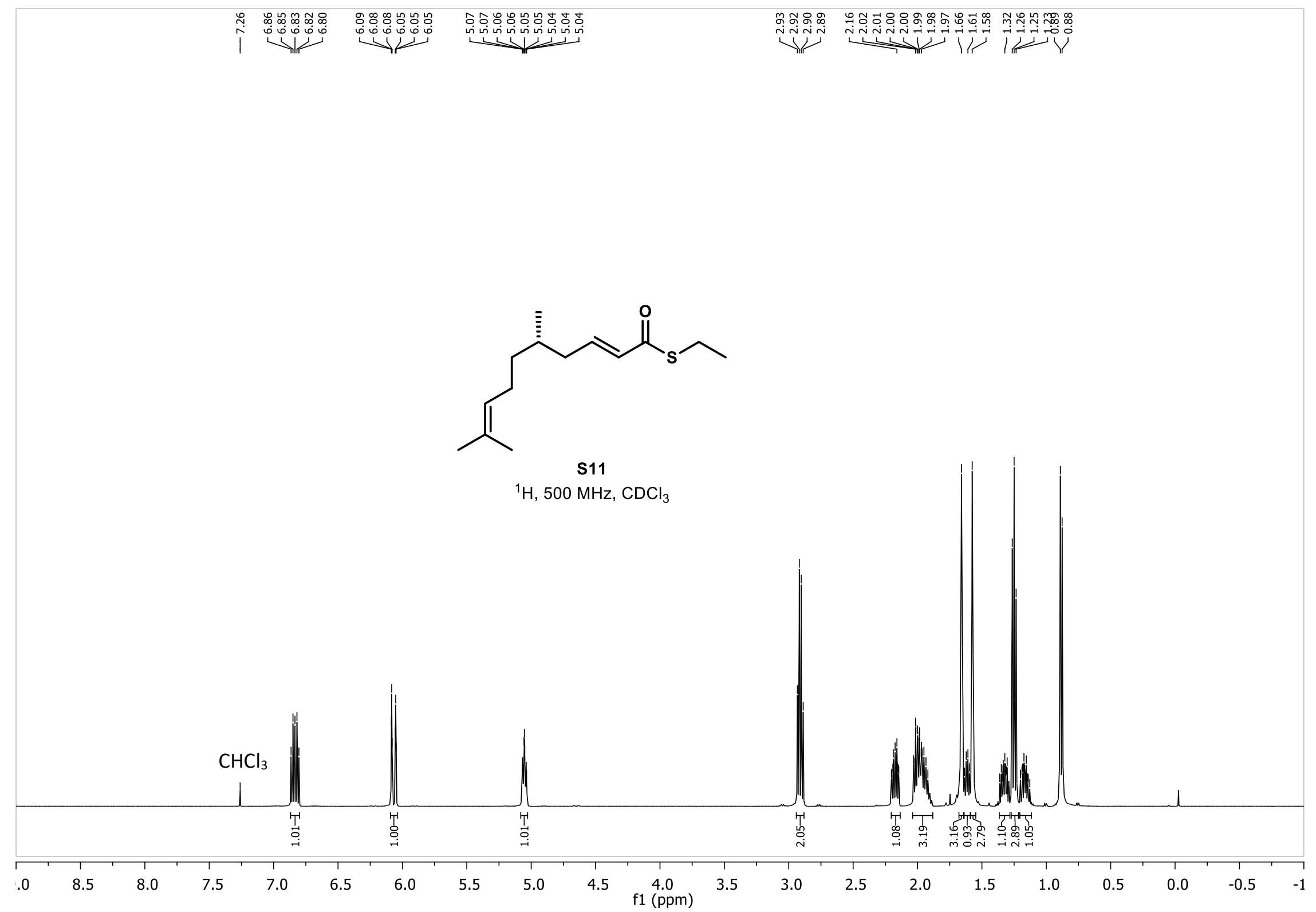




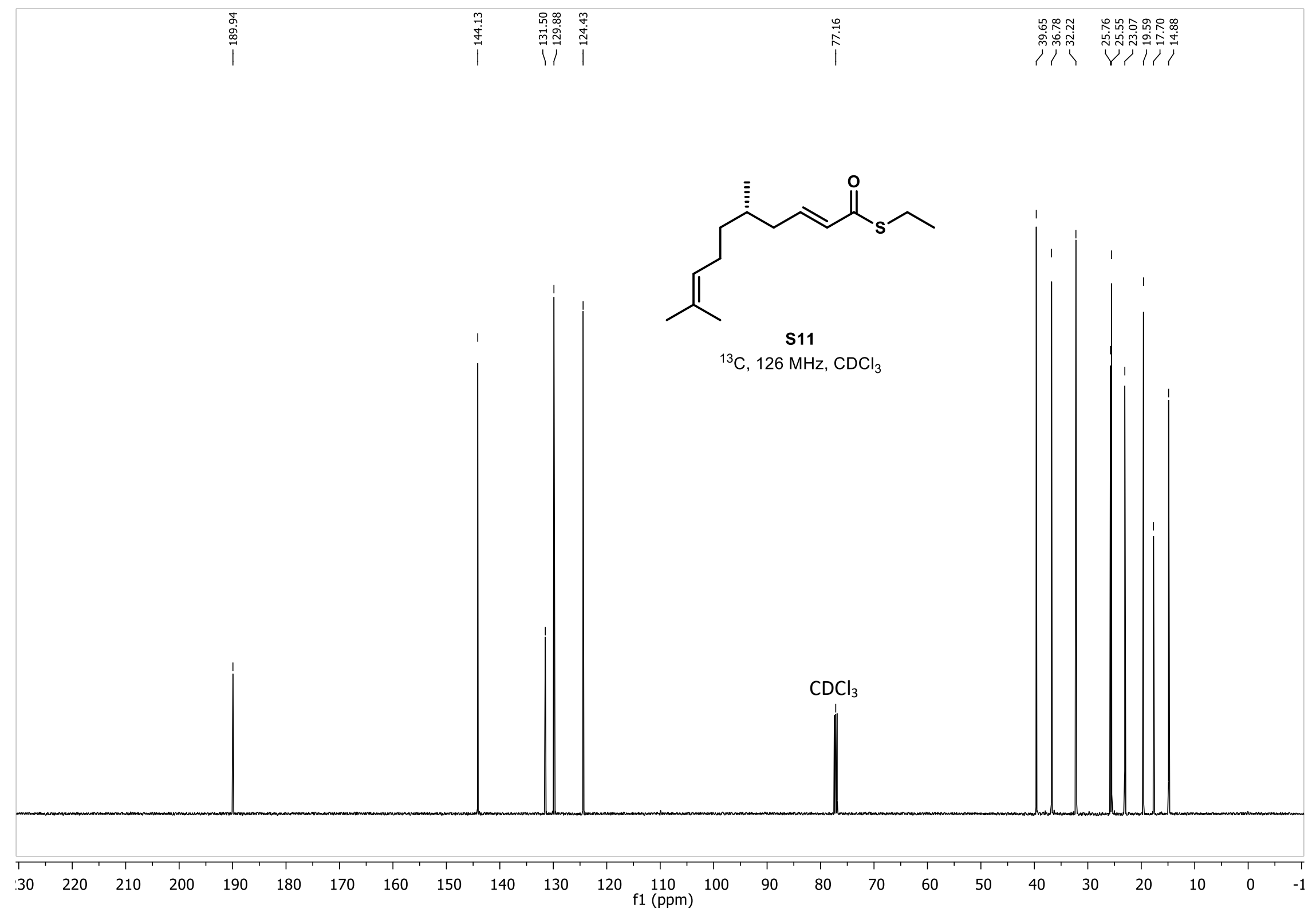




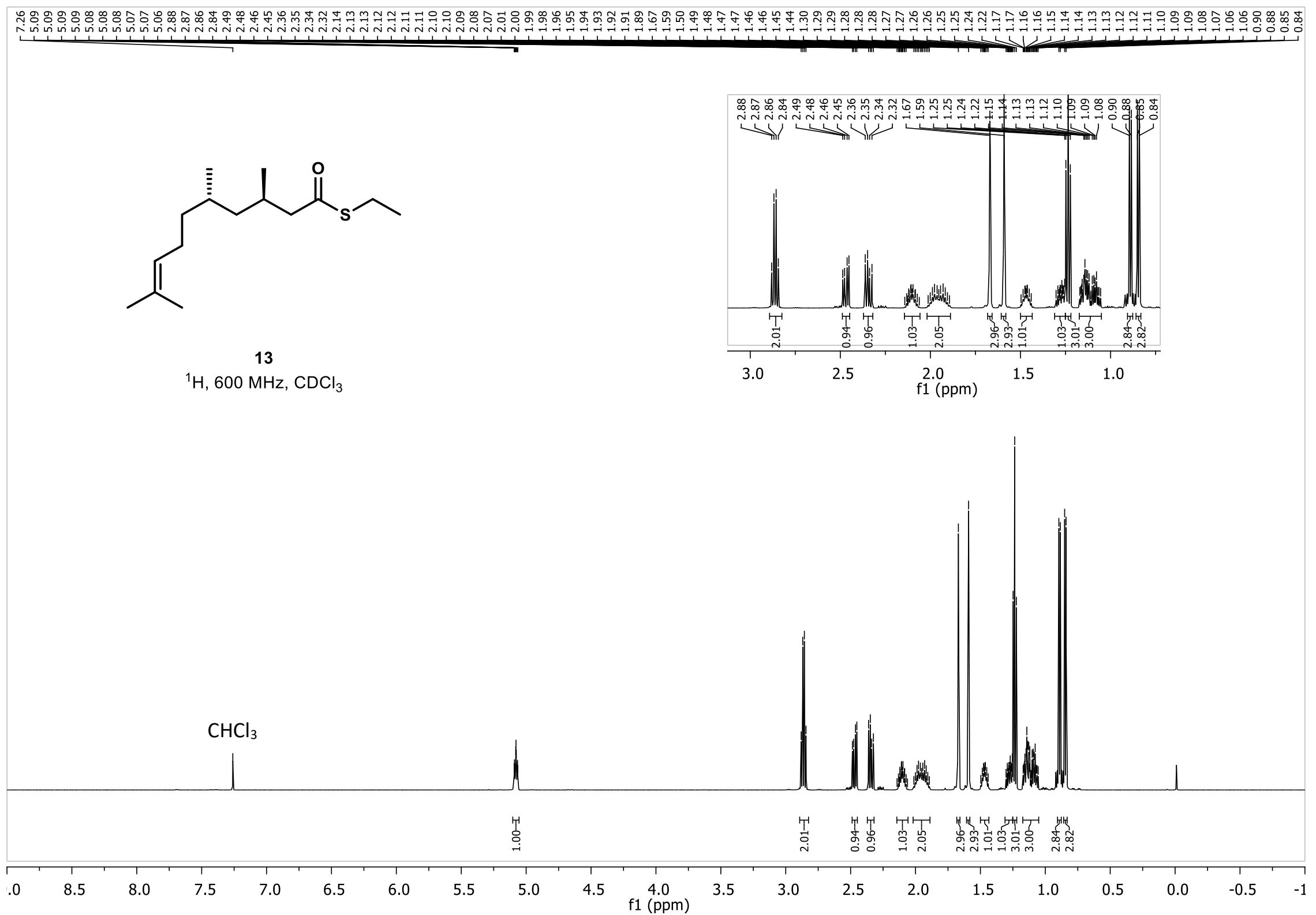



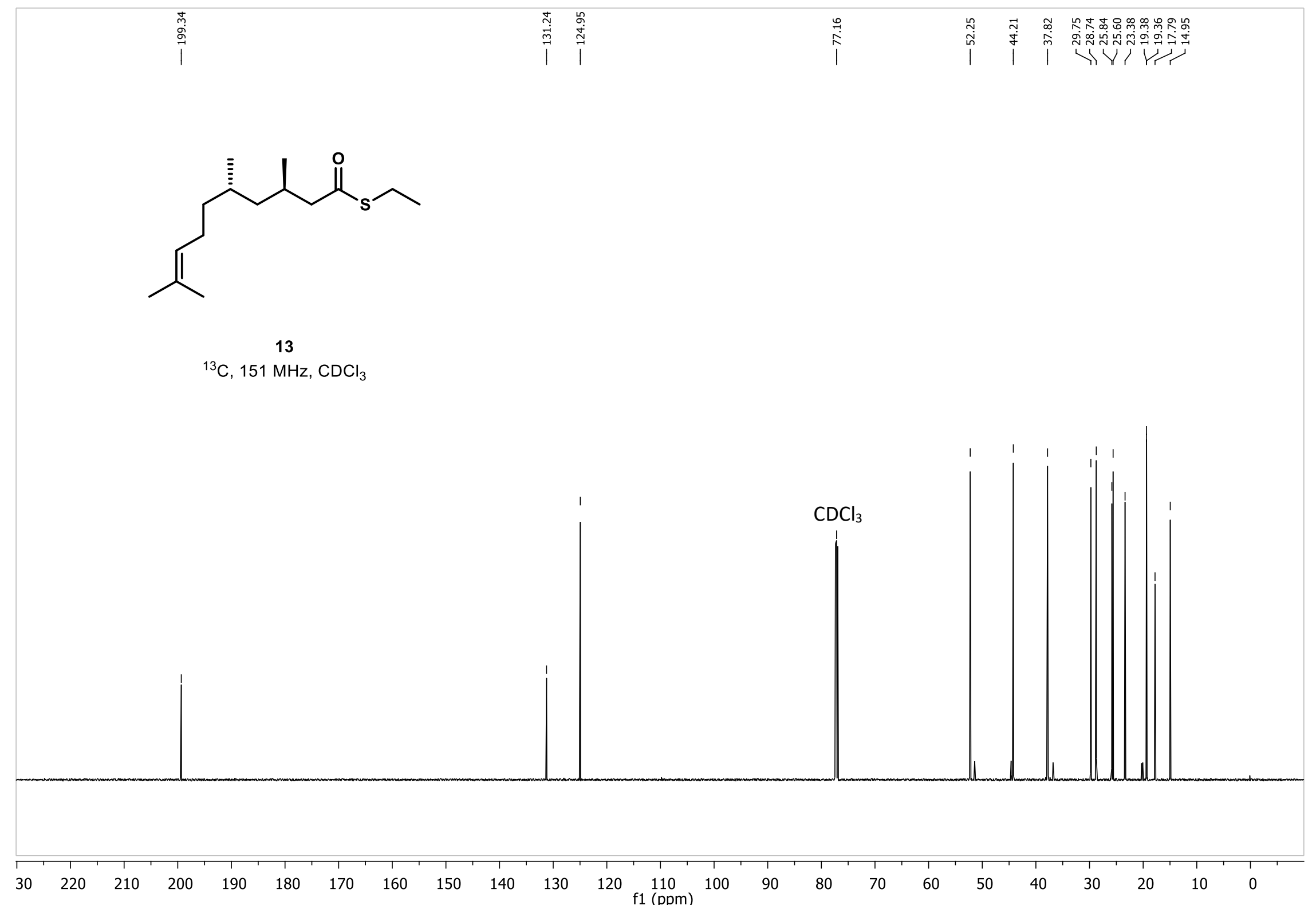


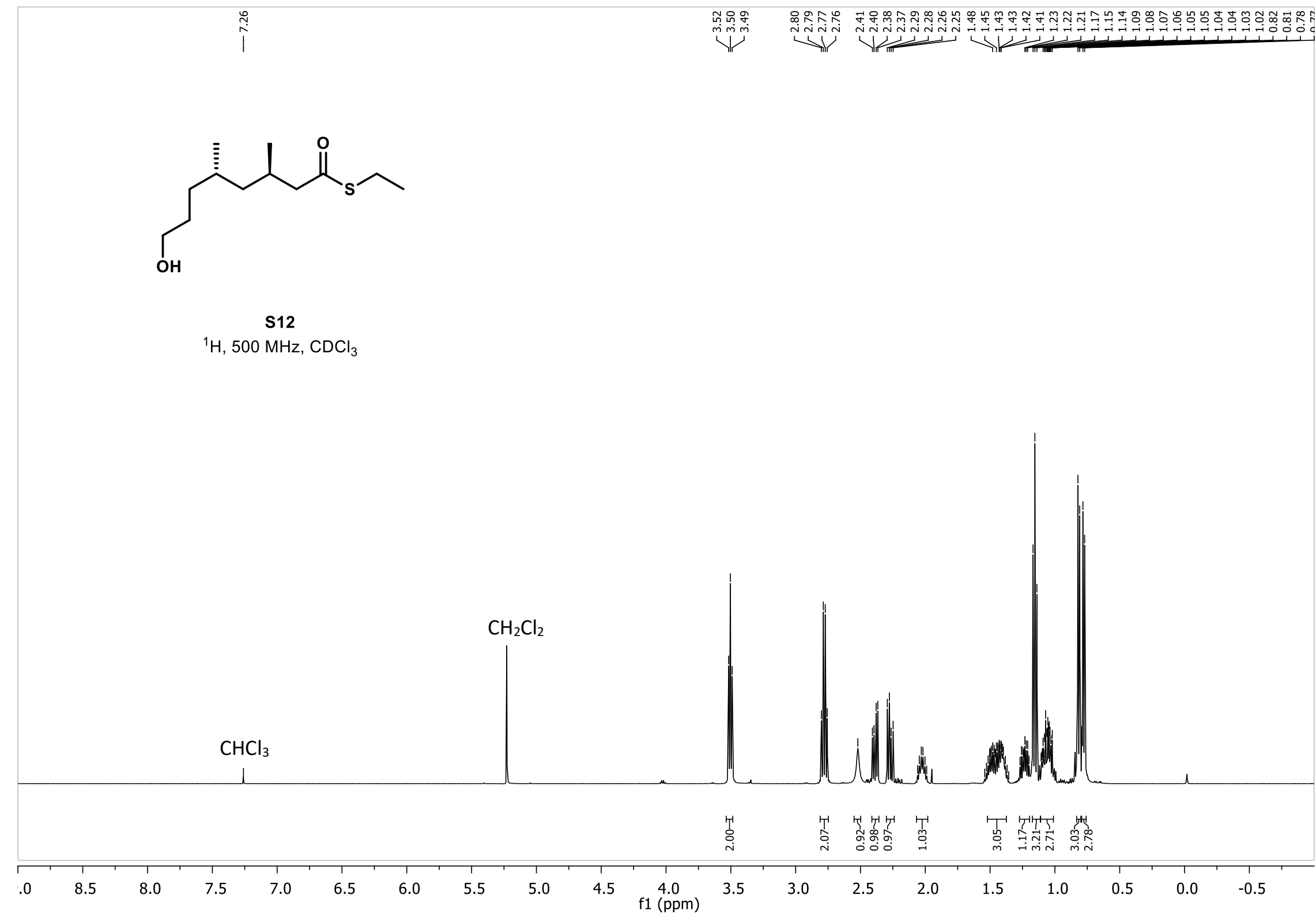




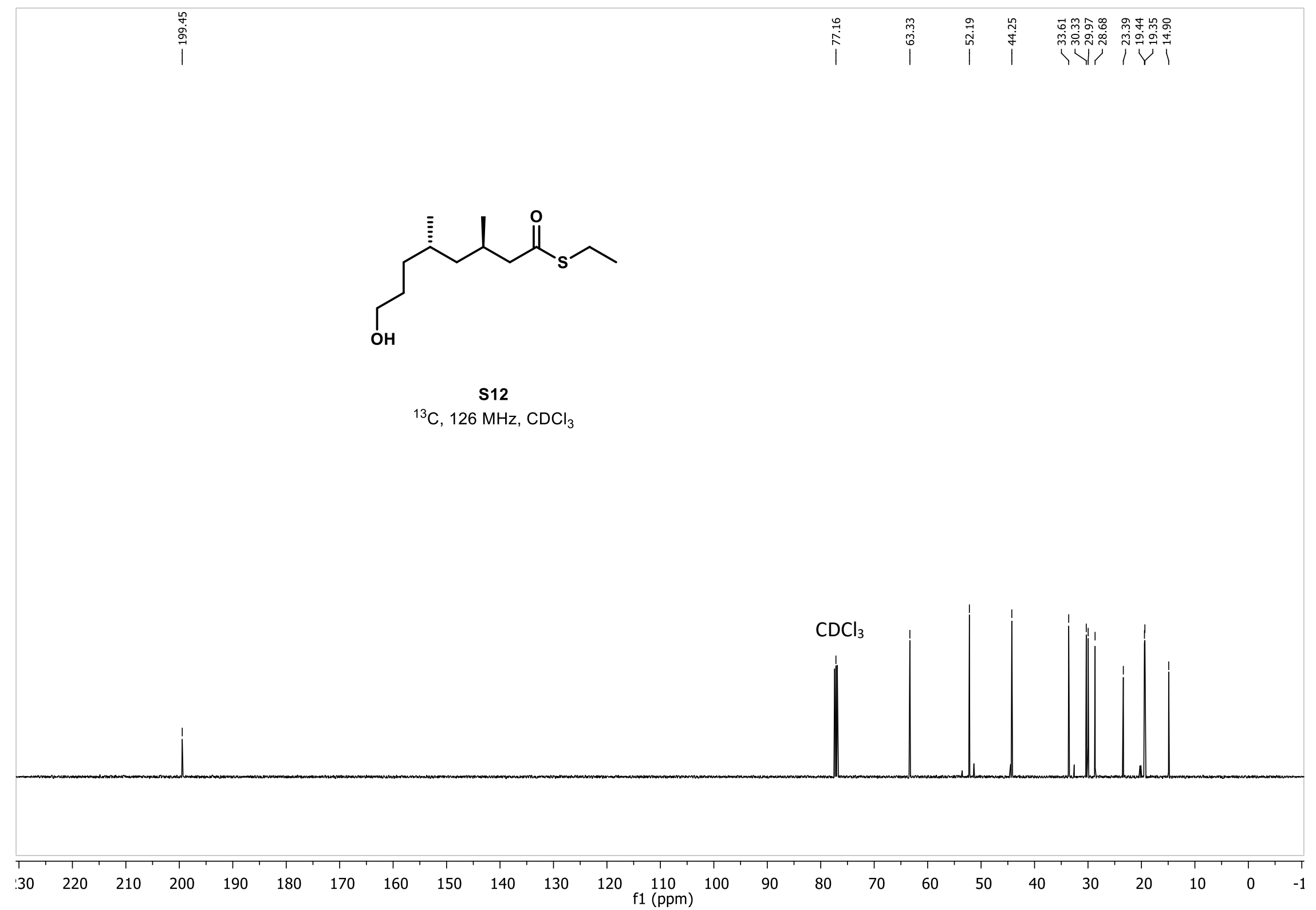




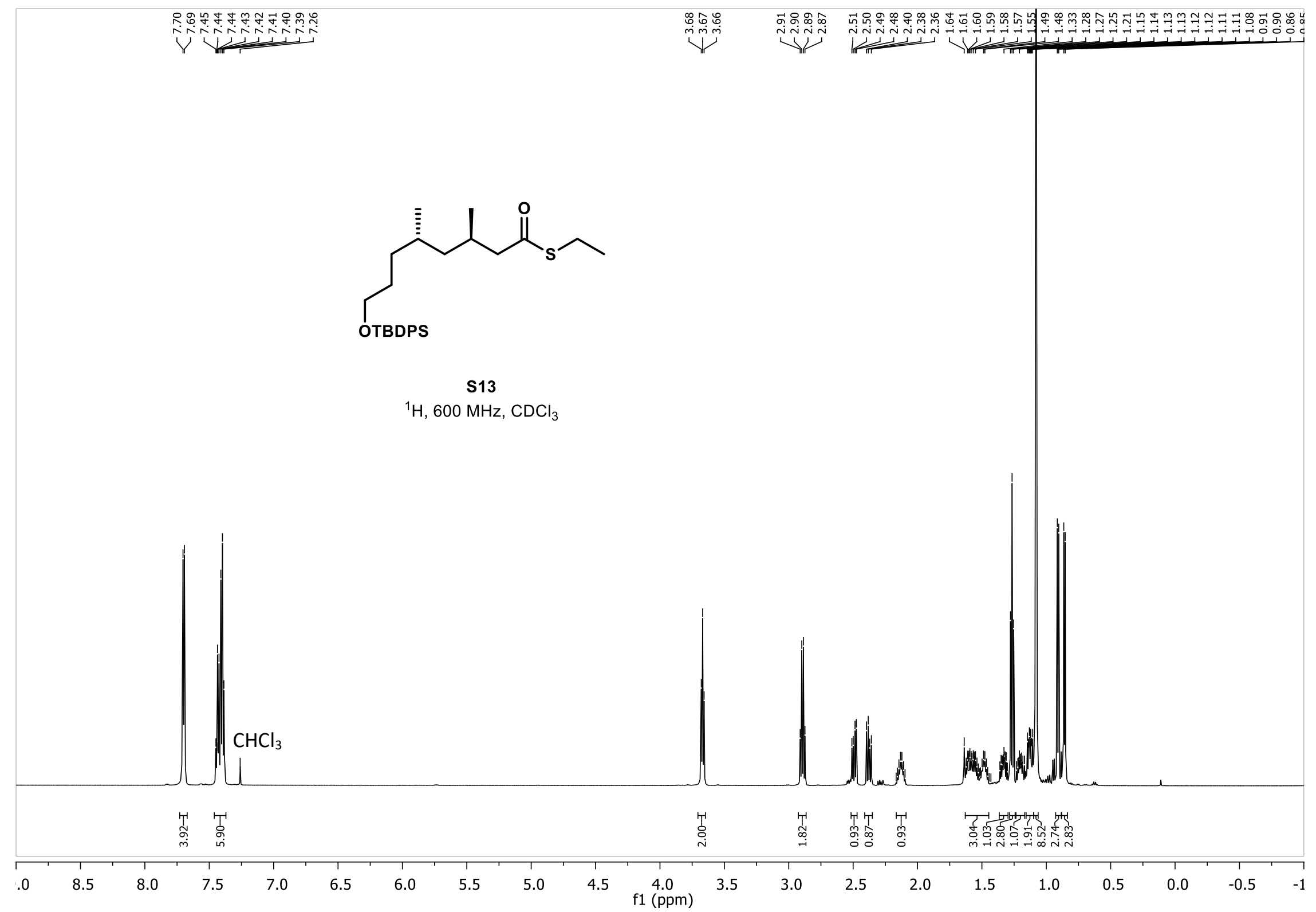




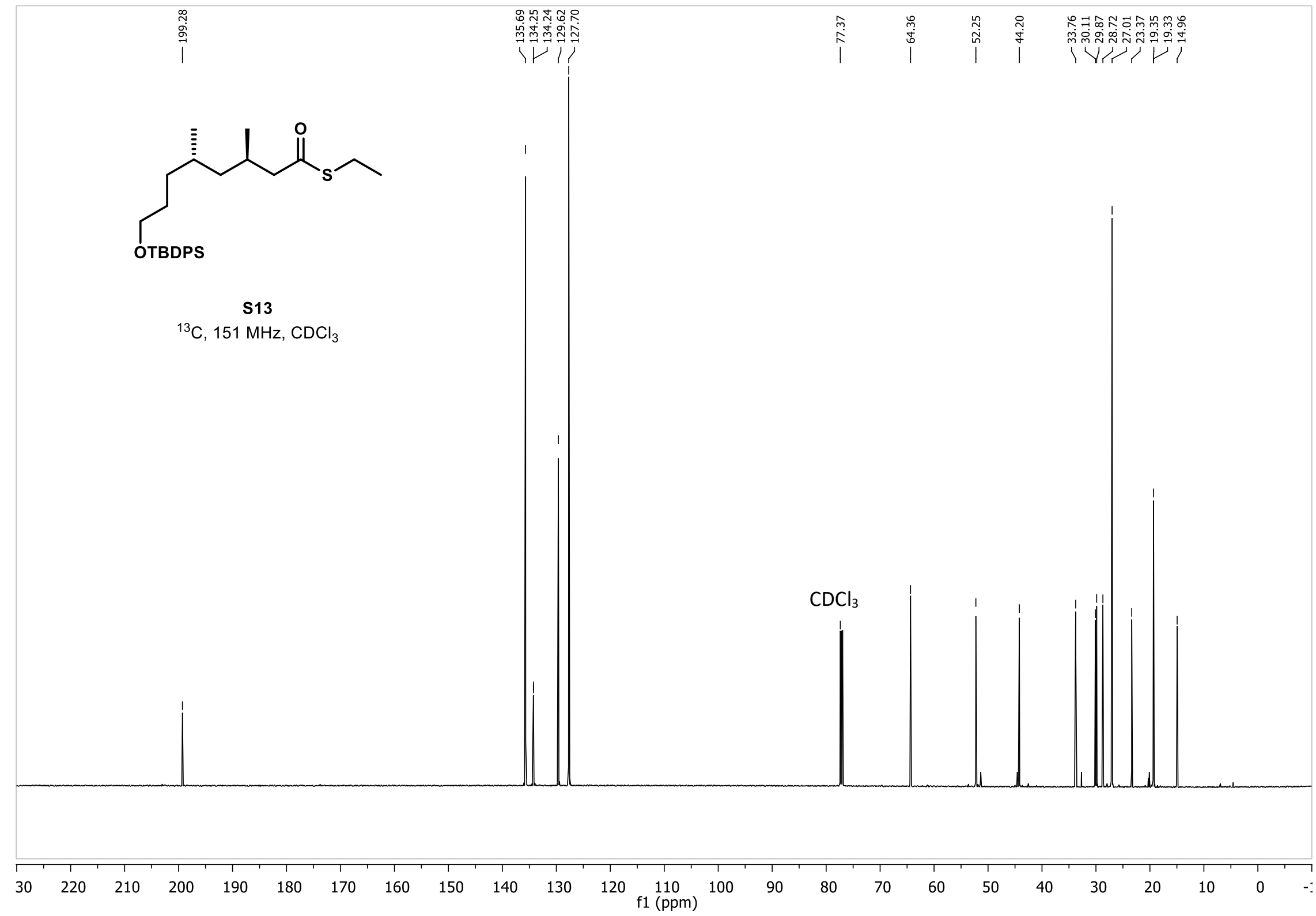




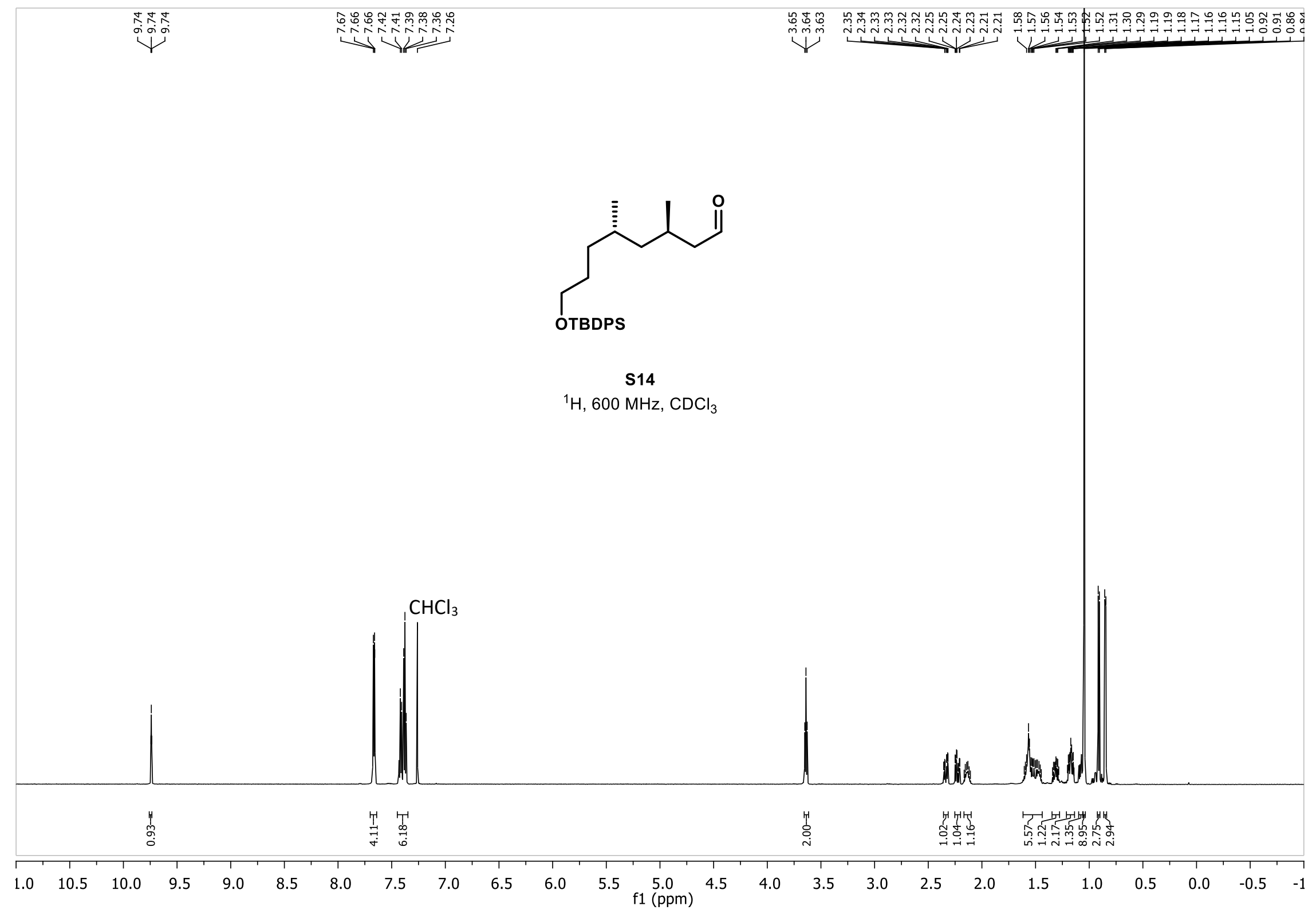




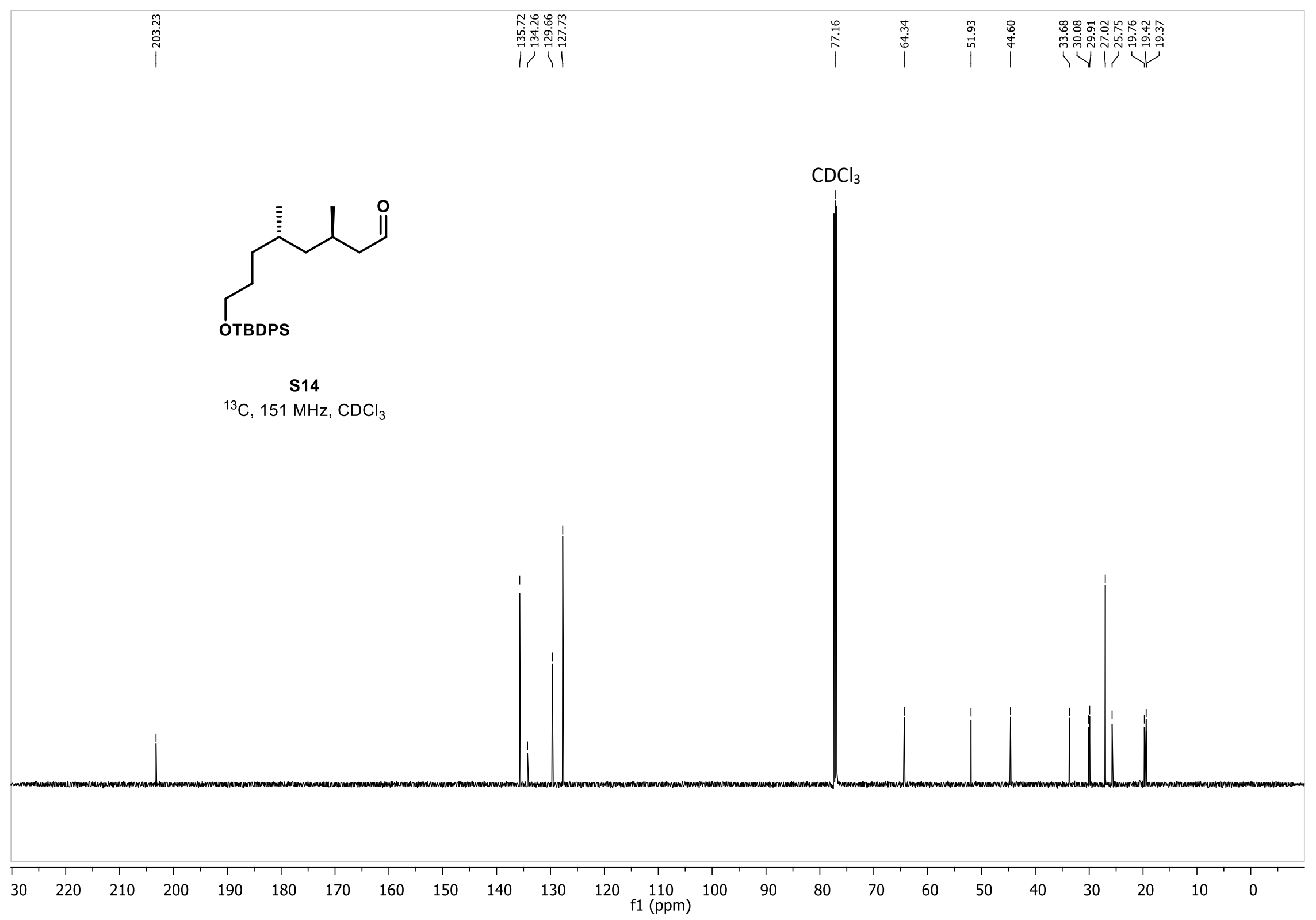

S58 


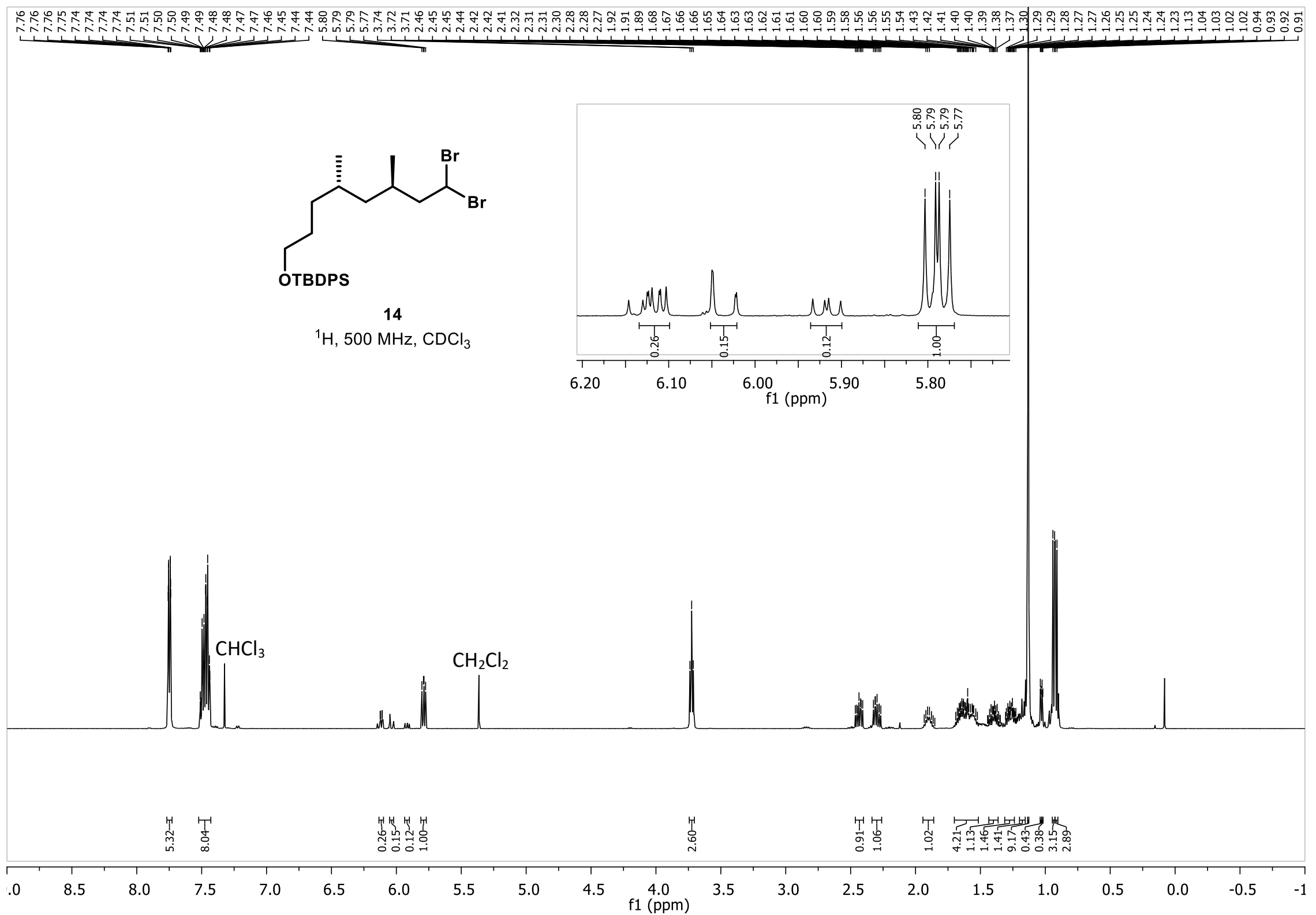




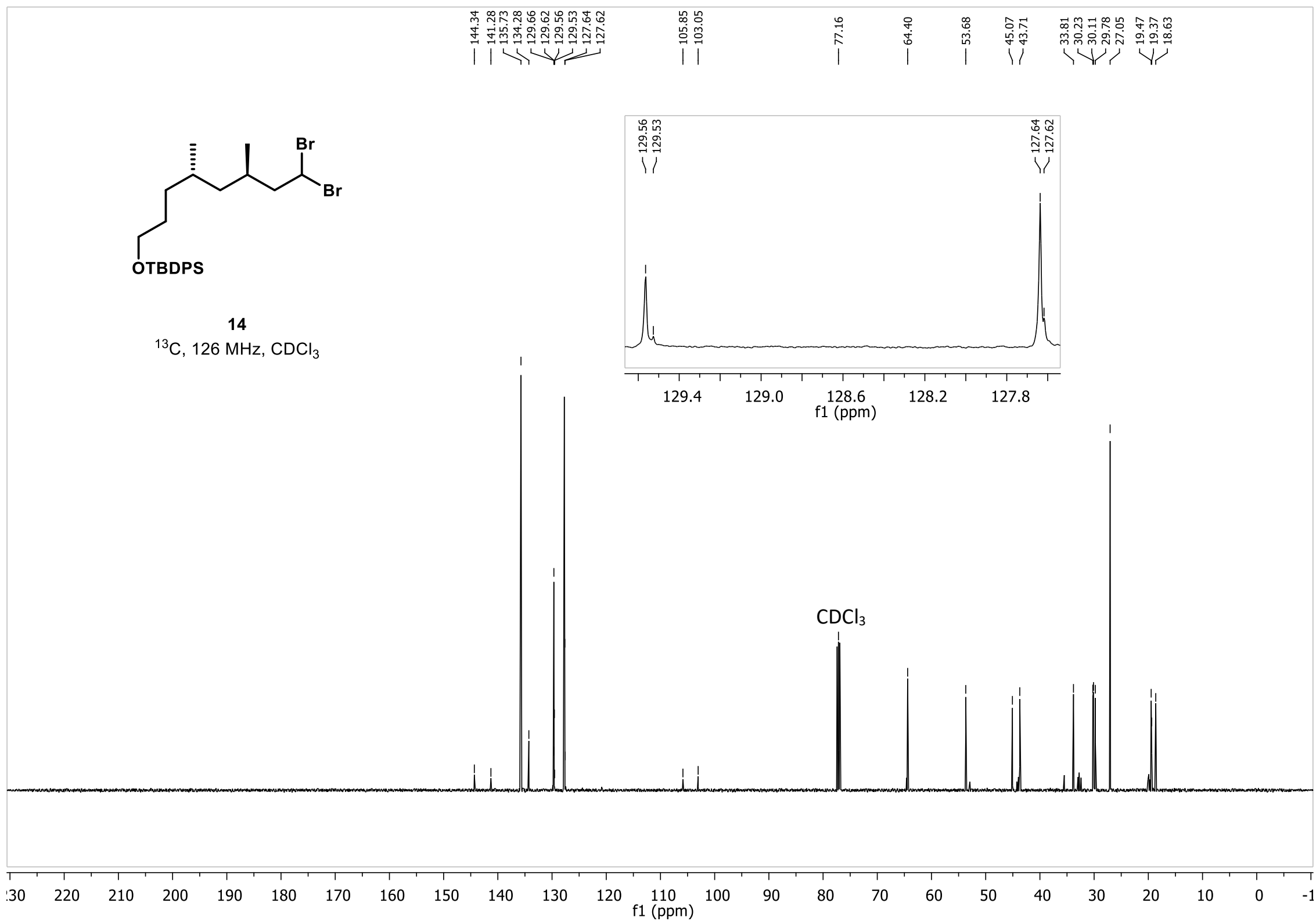




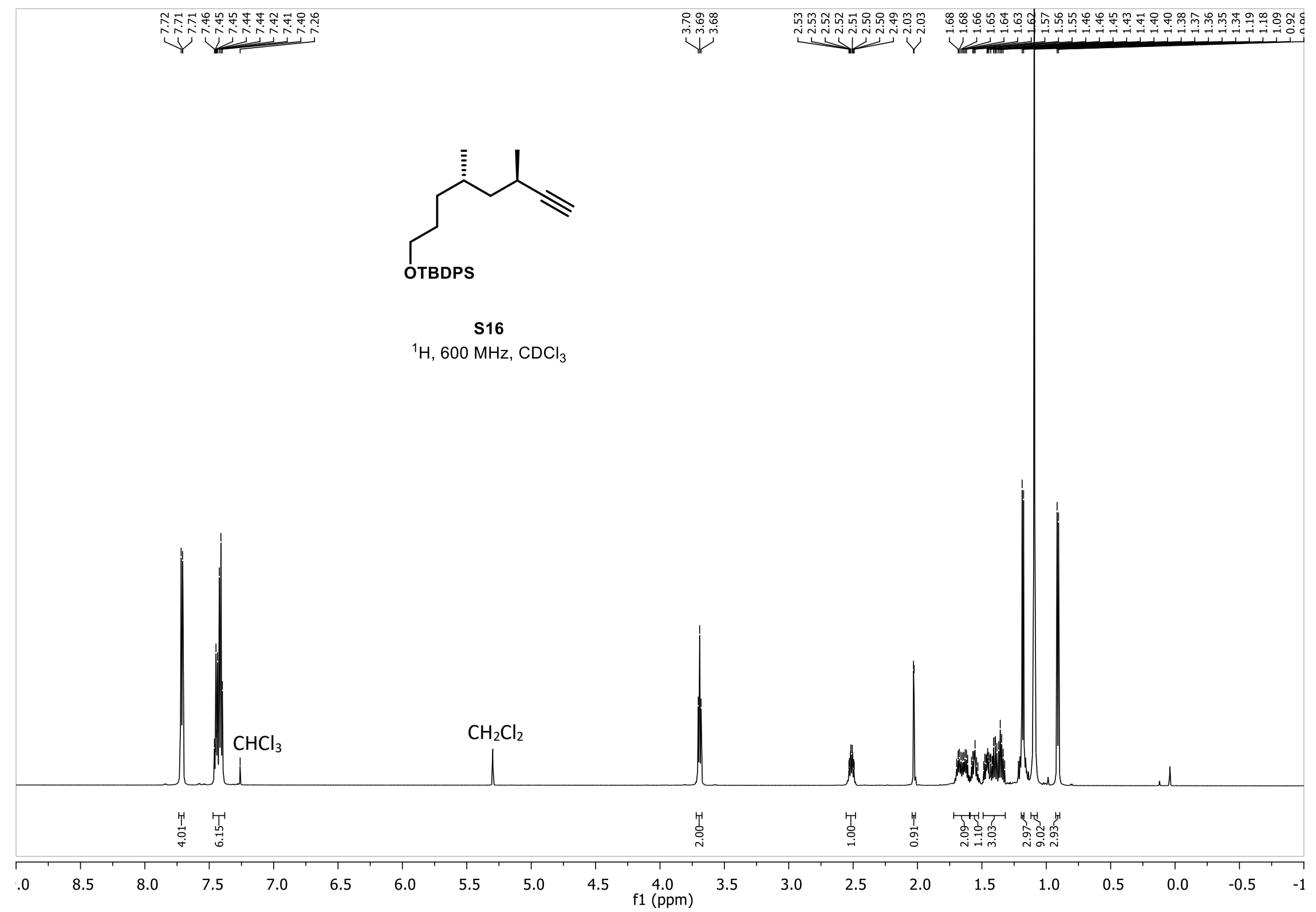




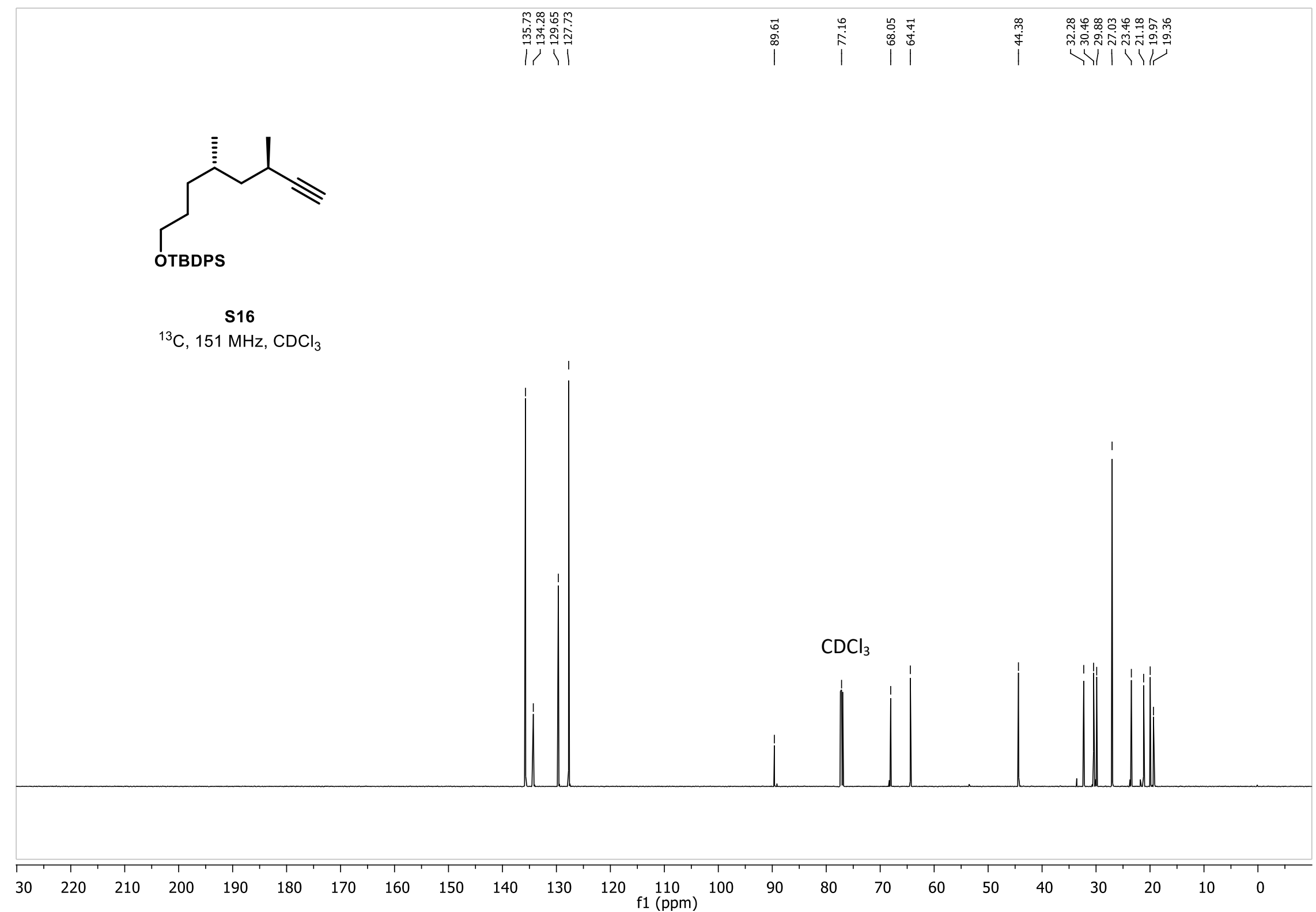




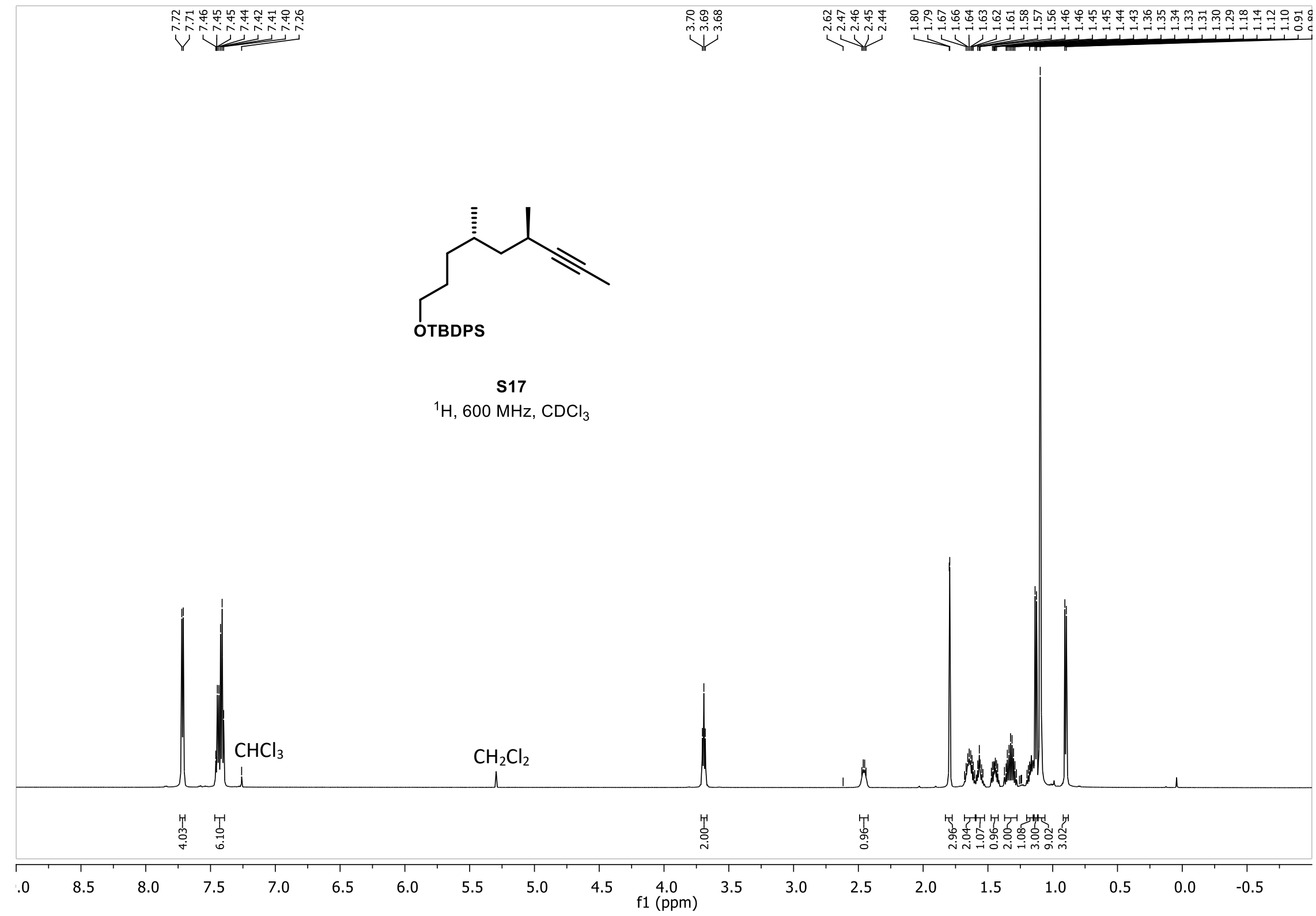




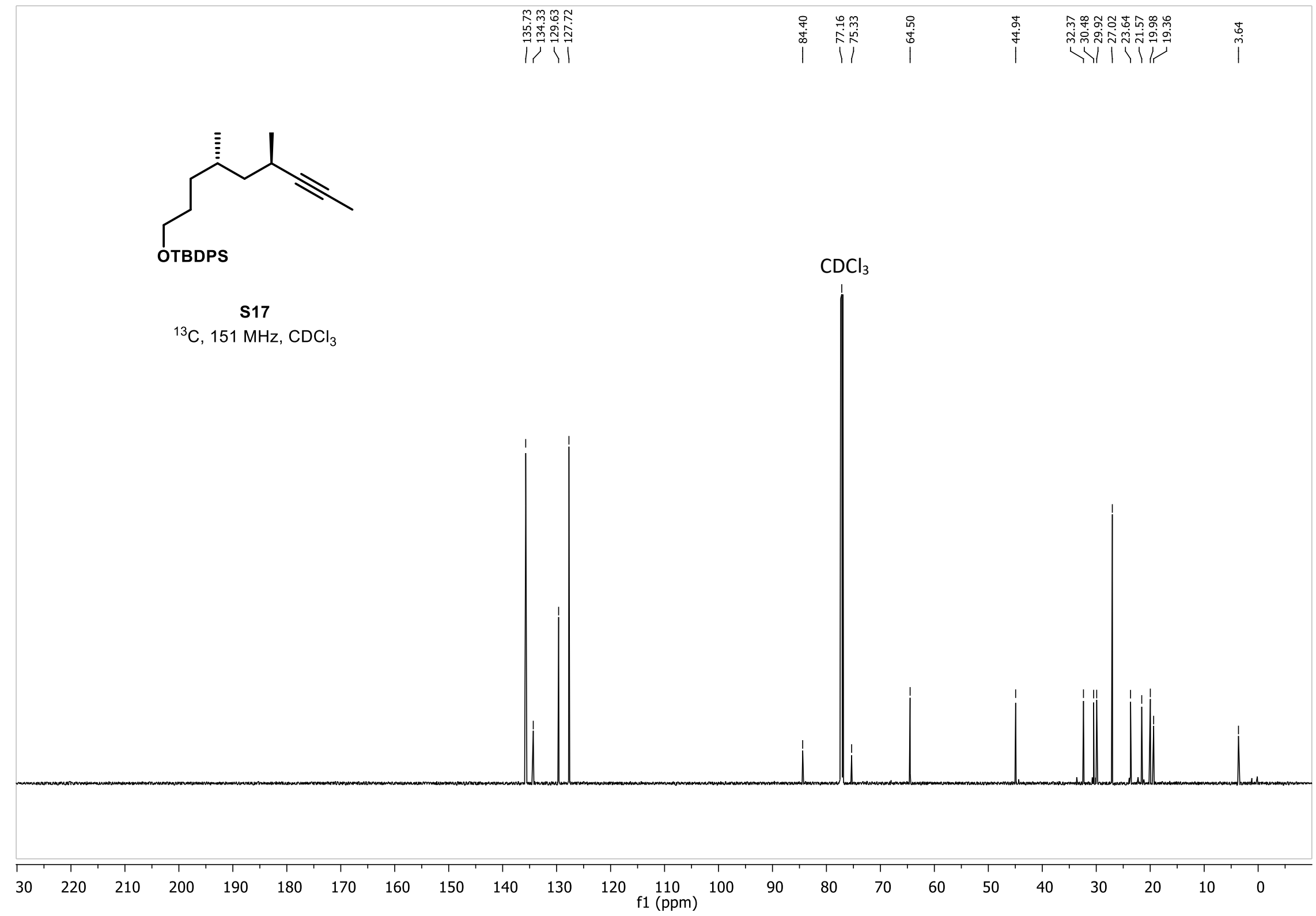




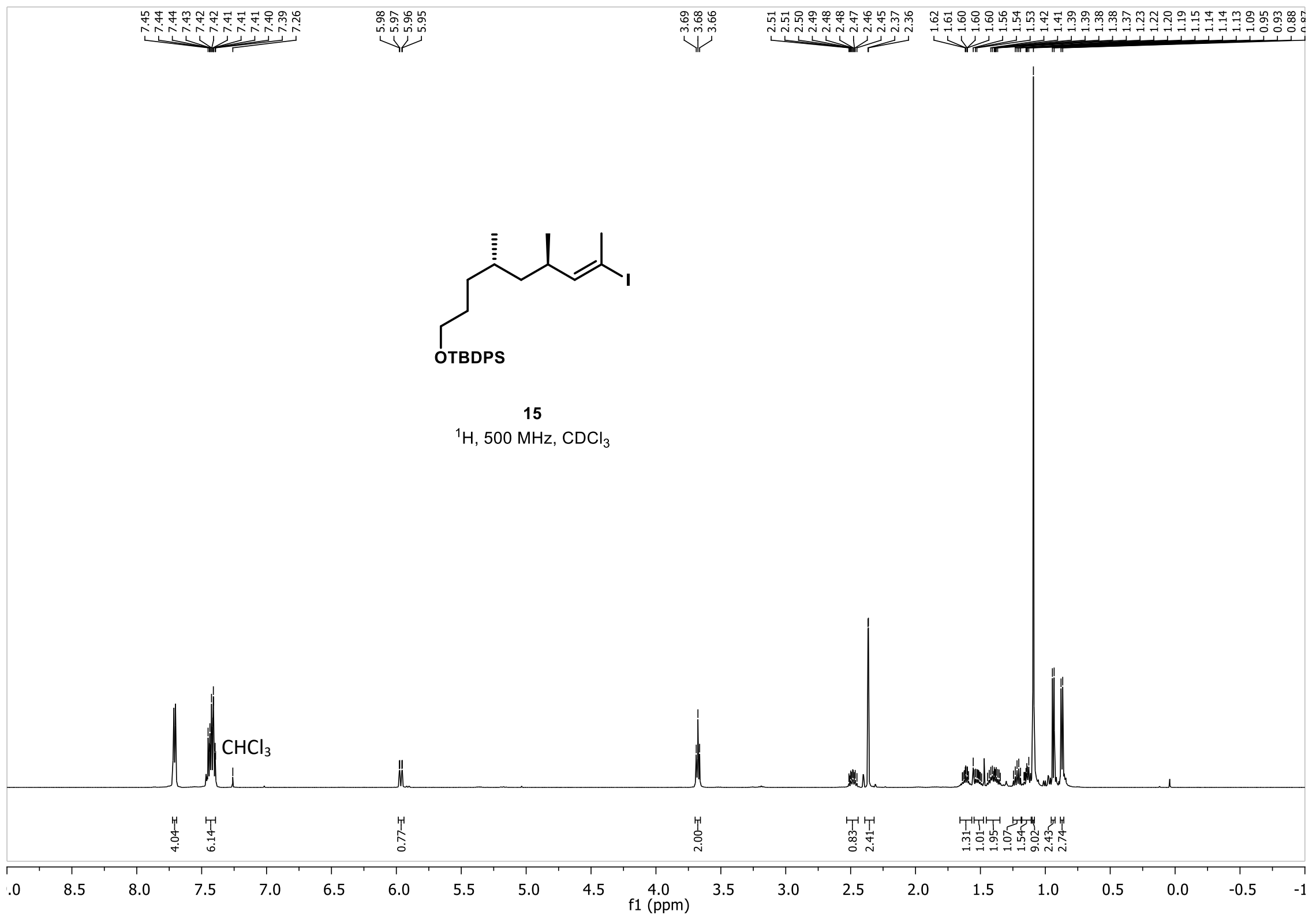




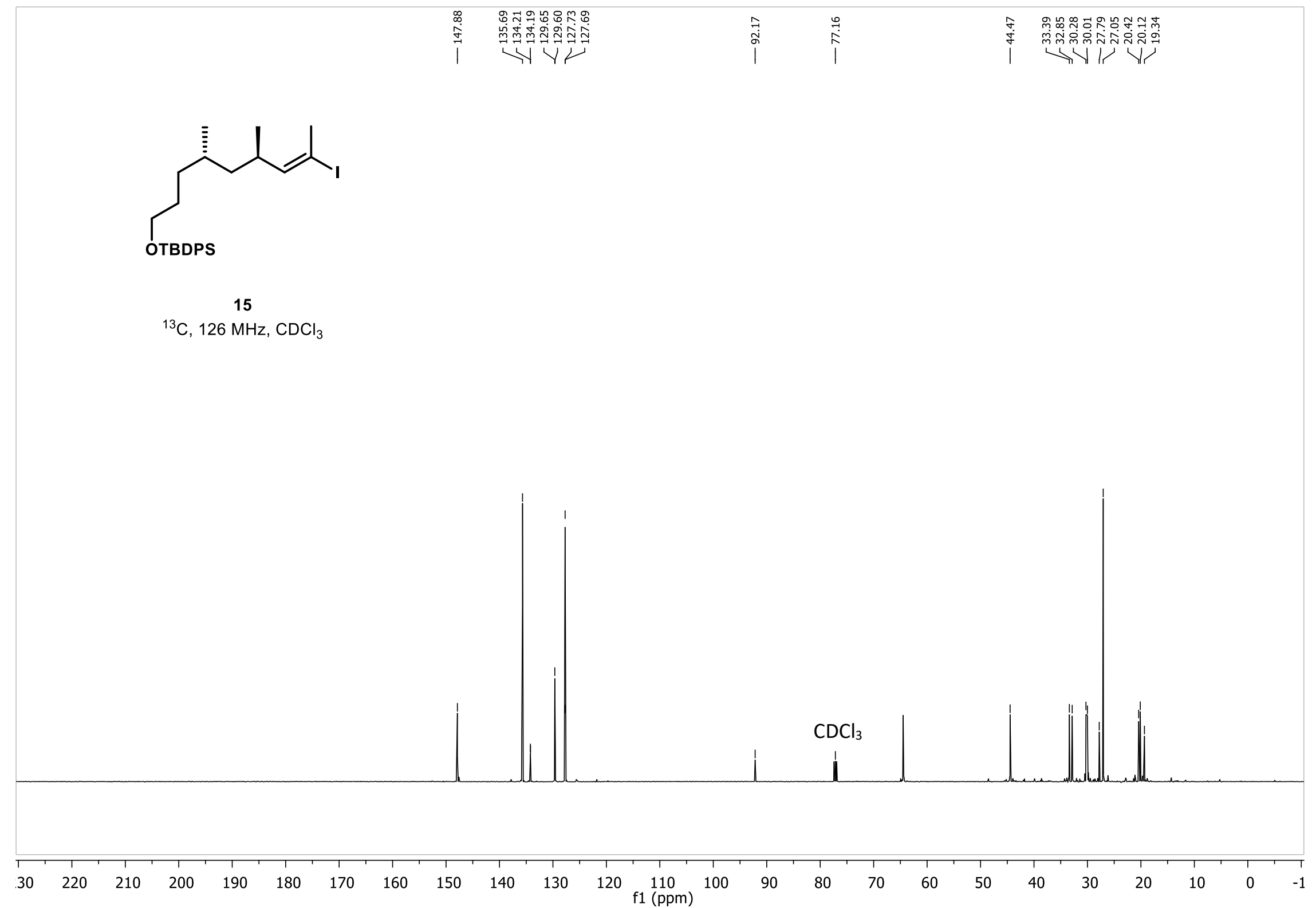




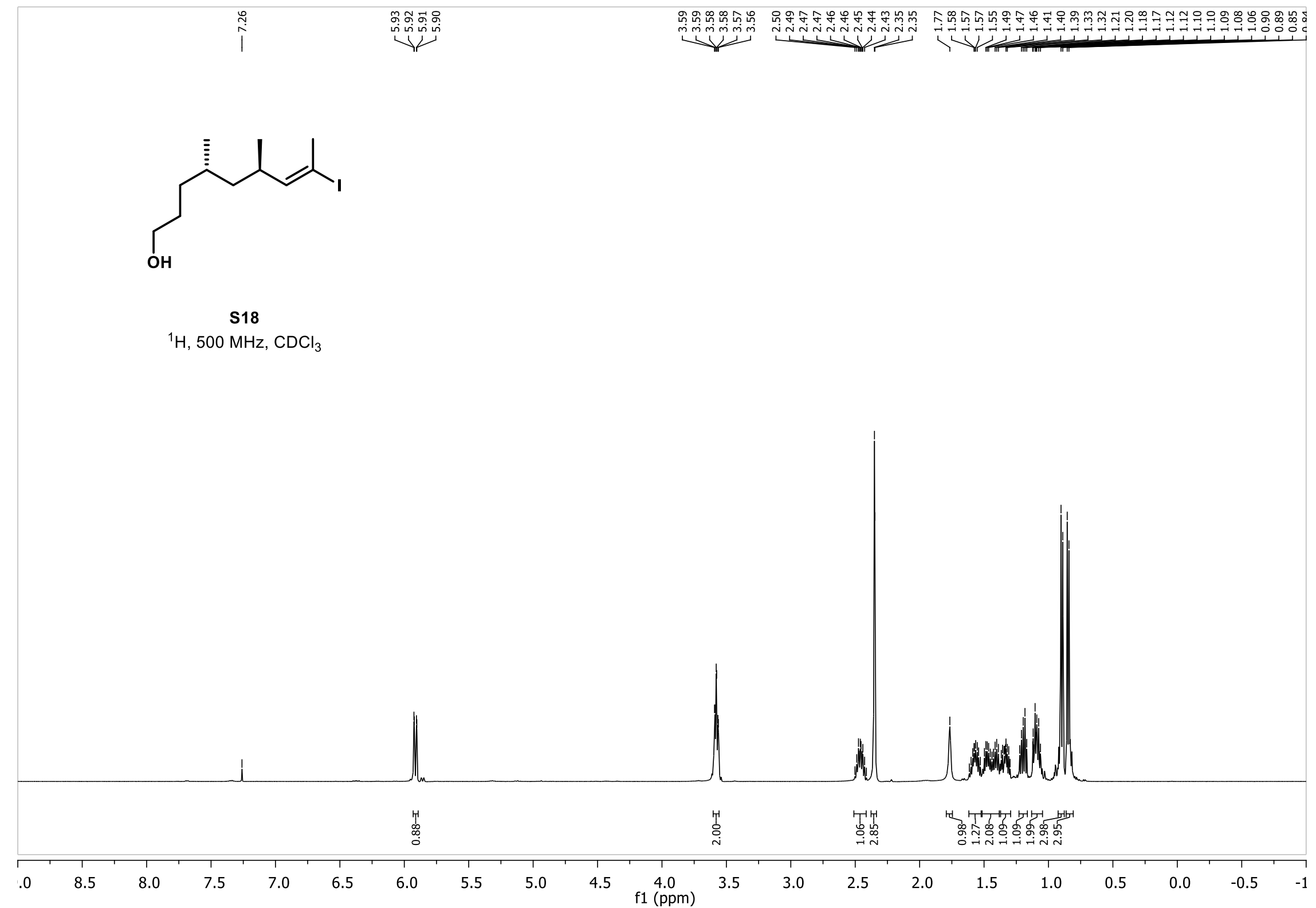




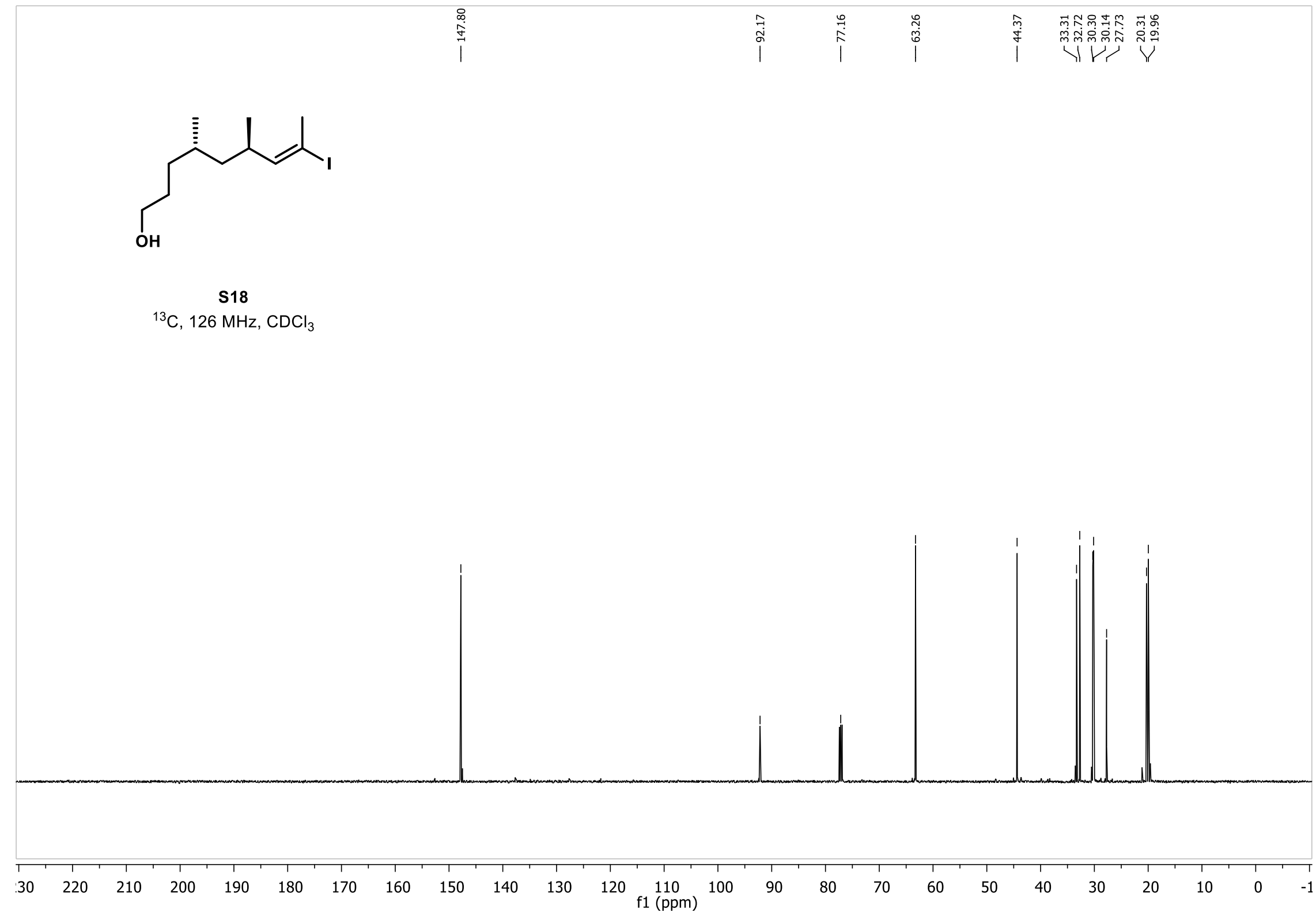




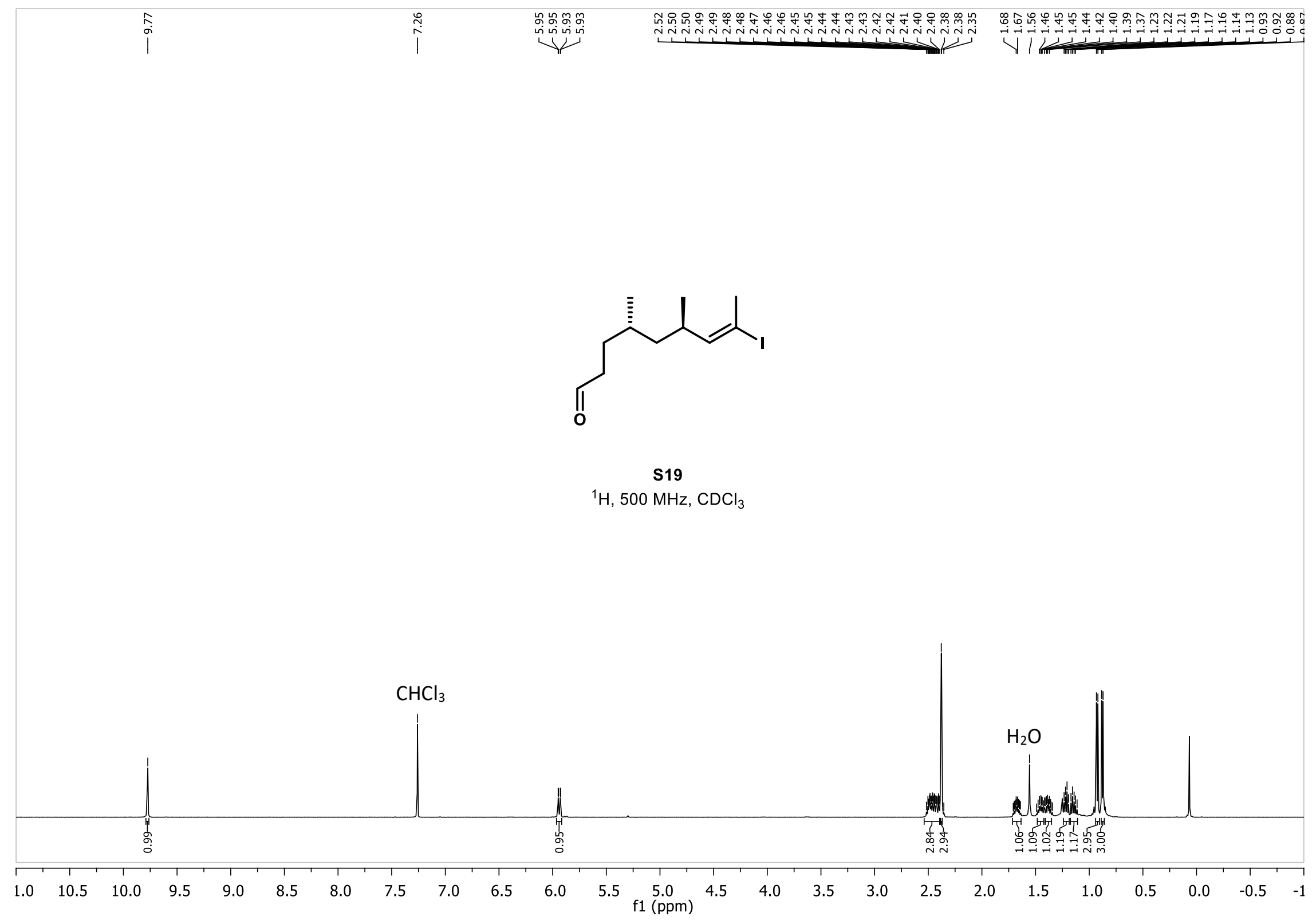



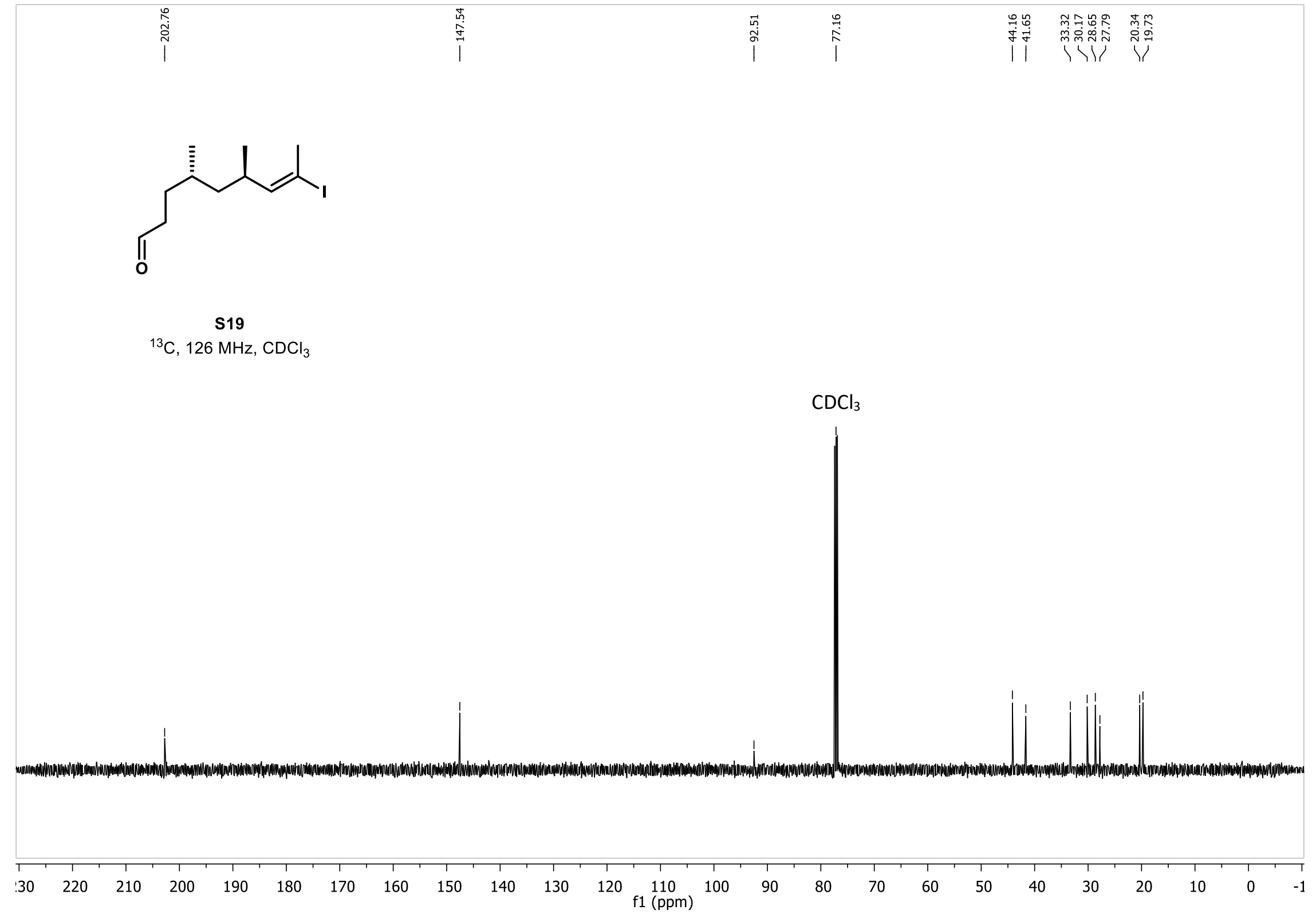


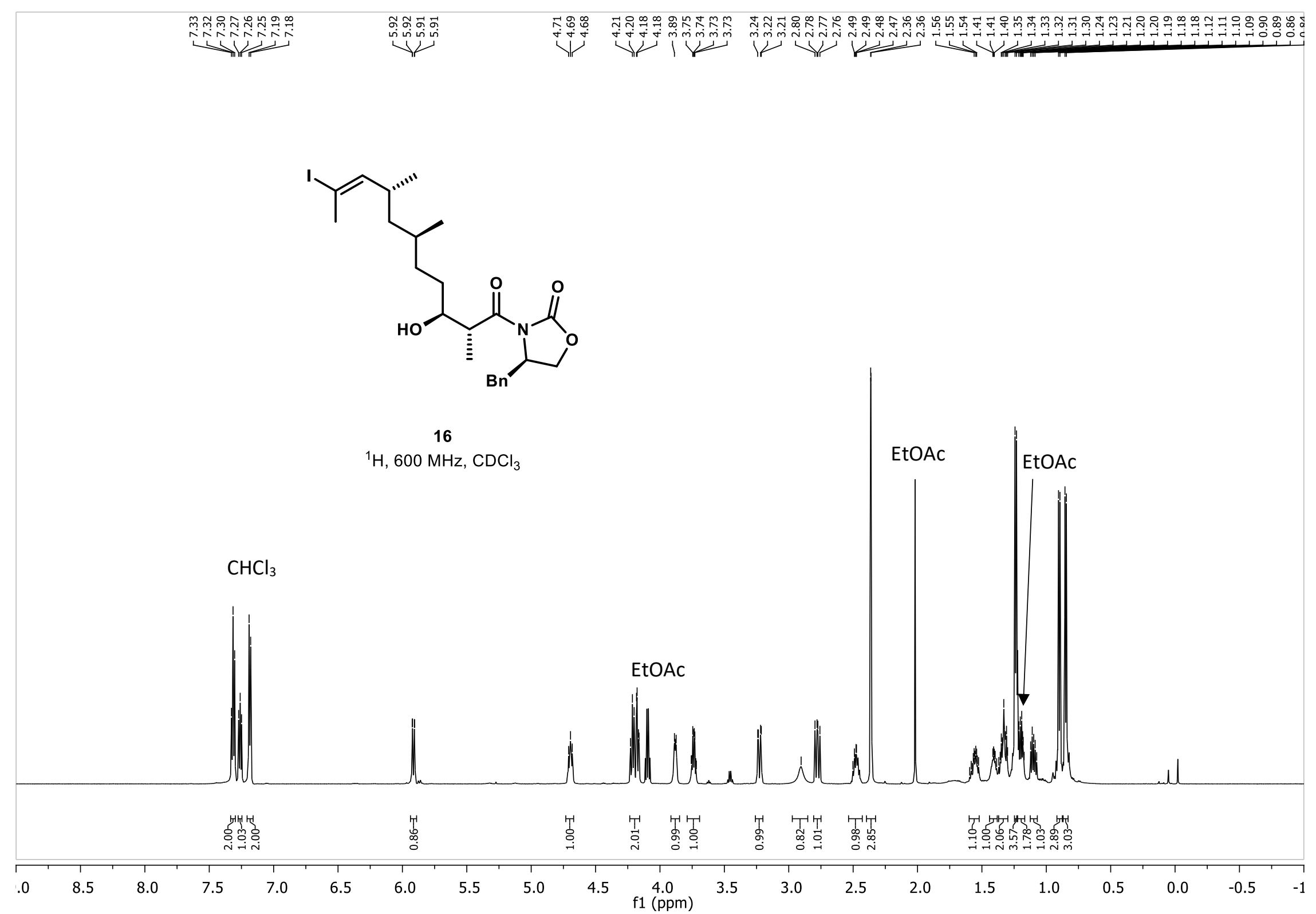




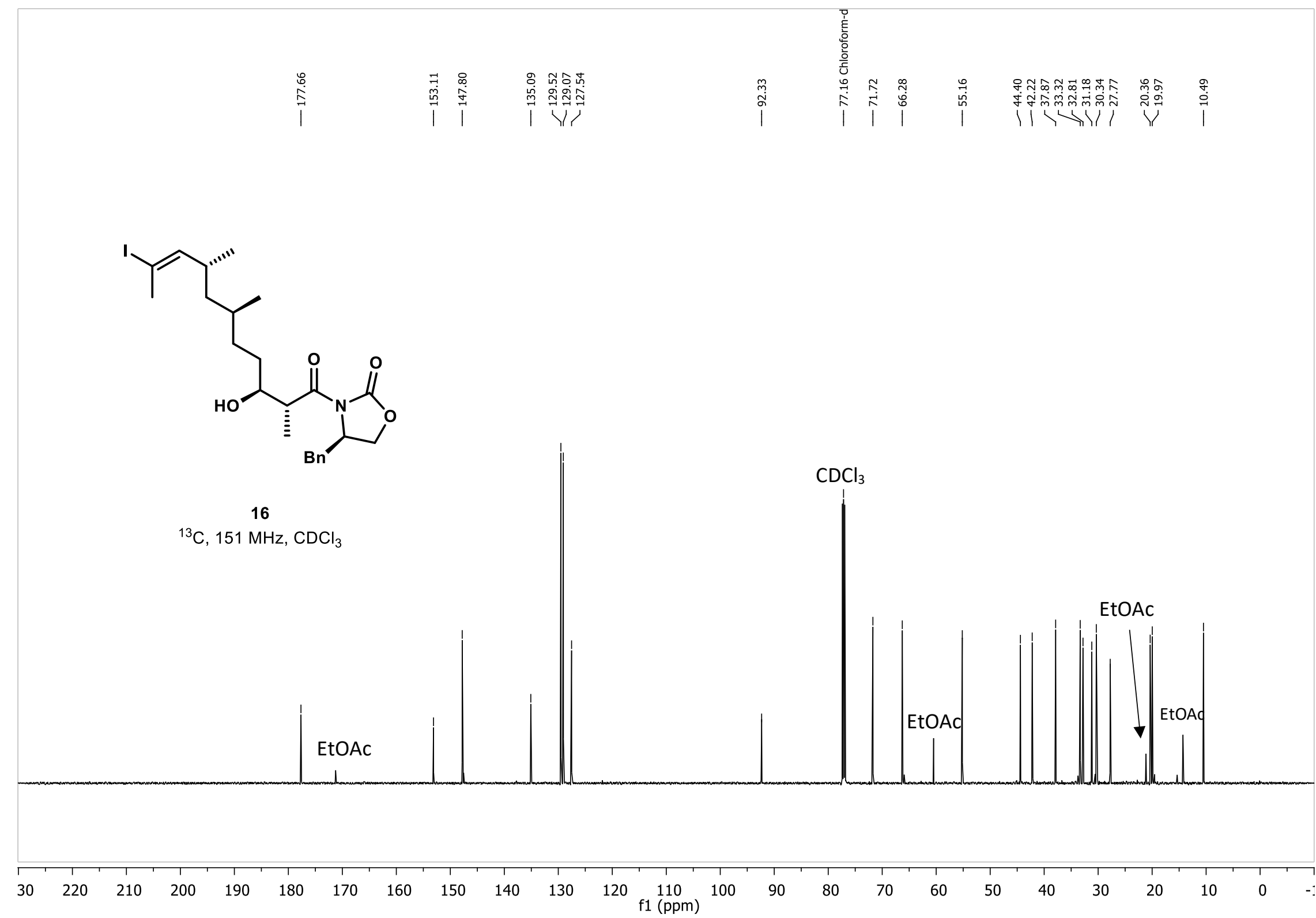




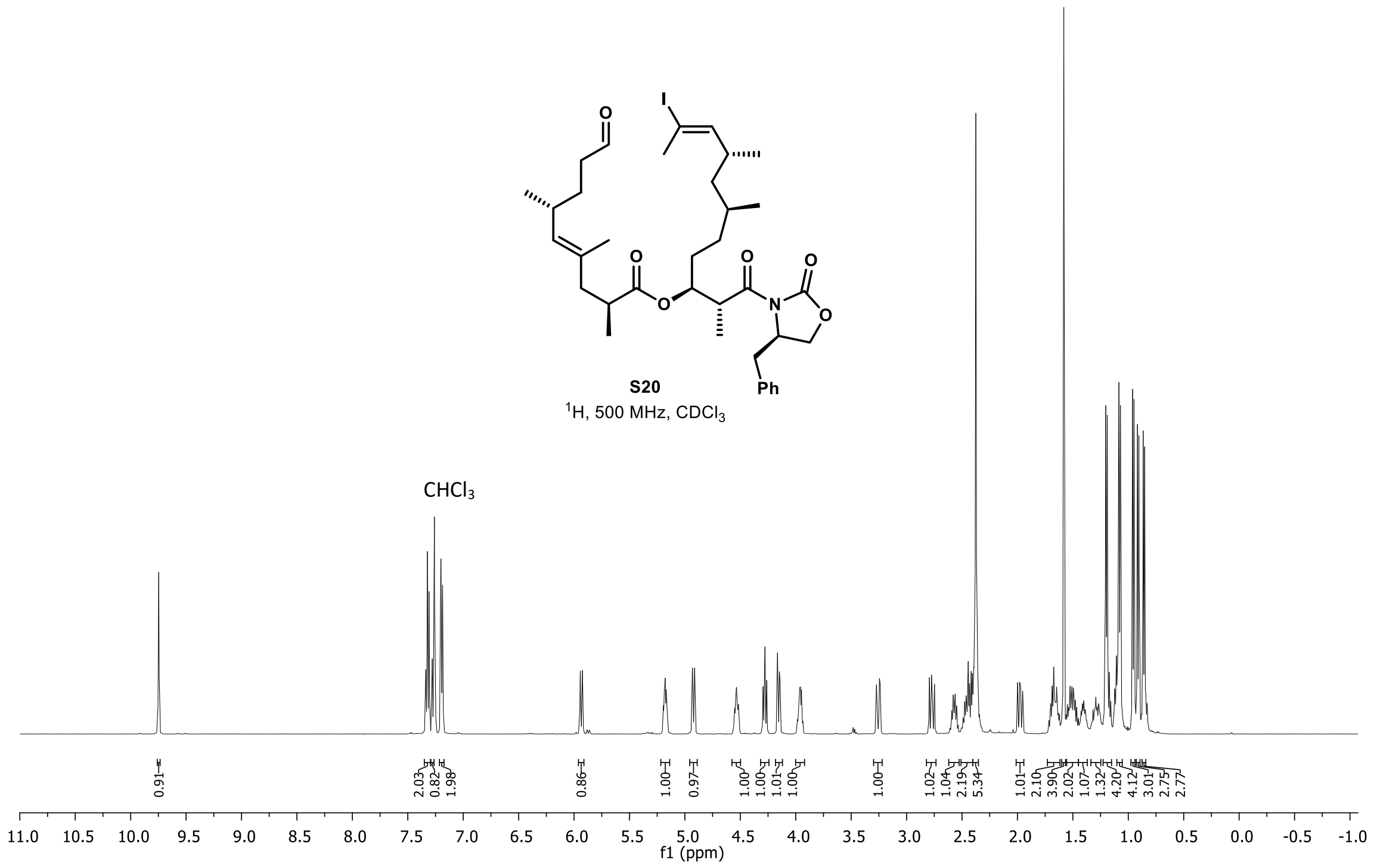




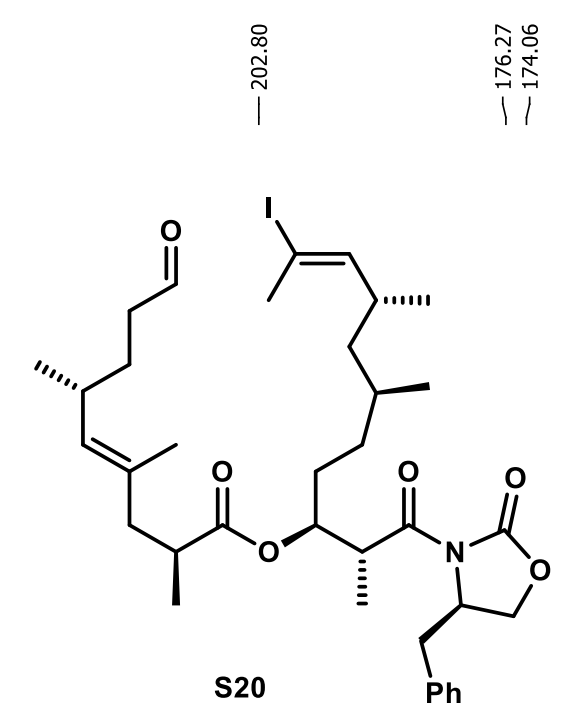

|

ले

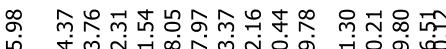

। ।

${ }^{13} \mathrm{C}, 126 \mathrm{MHz}, \mathrm{CDCl}_{3}$

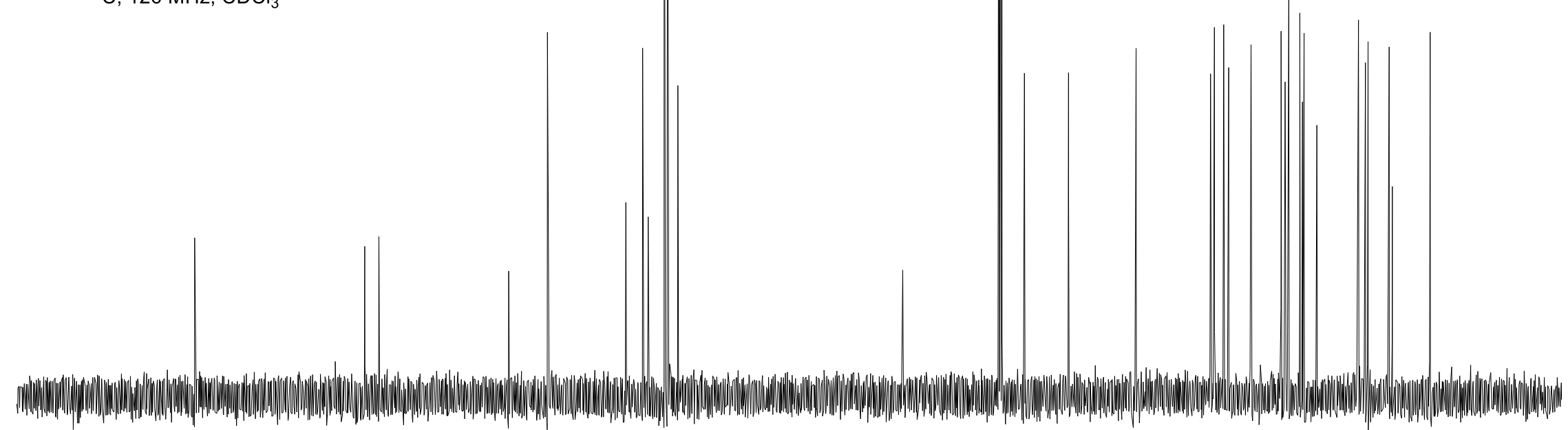

230

$\begin{array}{lllll}220 & 210 & 200 & 190 & 180\end{array}$

$170 \quad 160$

$150 \quad 140$

$120 \quad 110 \quad 100$

$90 \quad 80$

60

$50 \quad 40$

30

$20 \quad 10$

$-10$ 


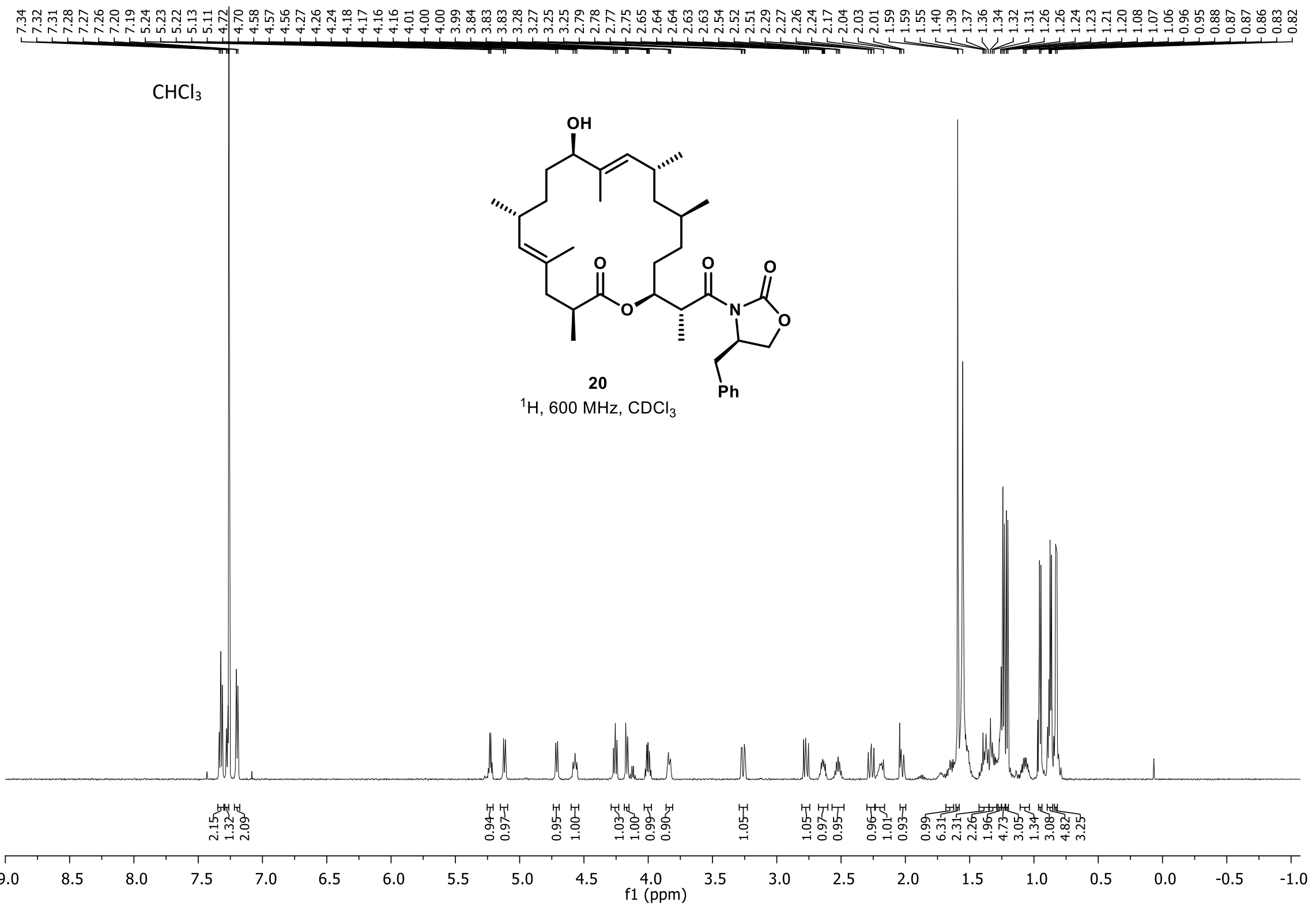




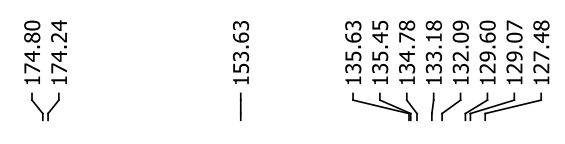

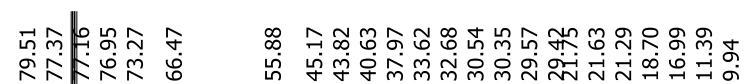

$\underbrace{2}$

$\mathrm{CDCl}_{3}$

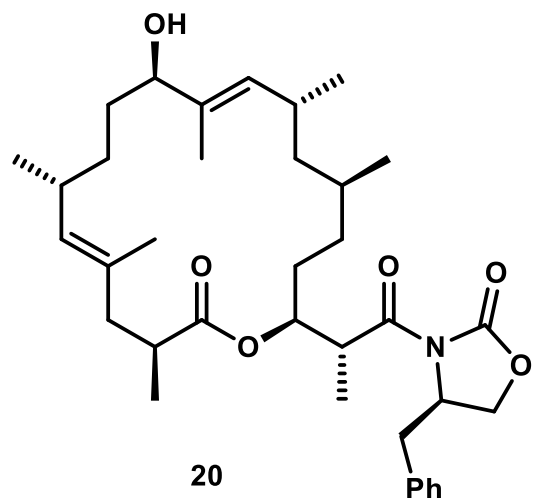

${ }^{13} \mathrm{C}, 151 \mathrm{MHz}, \mathrm{CDCl}_{3}$

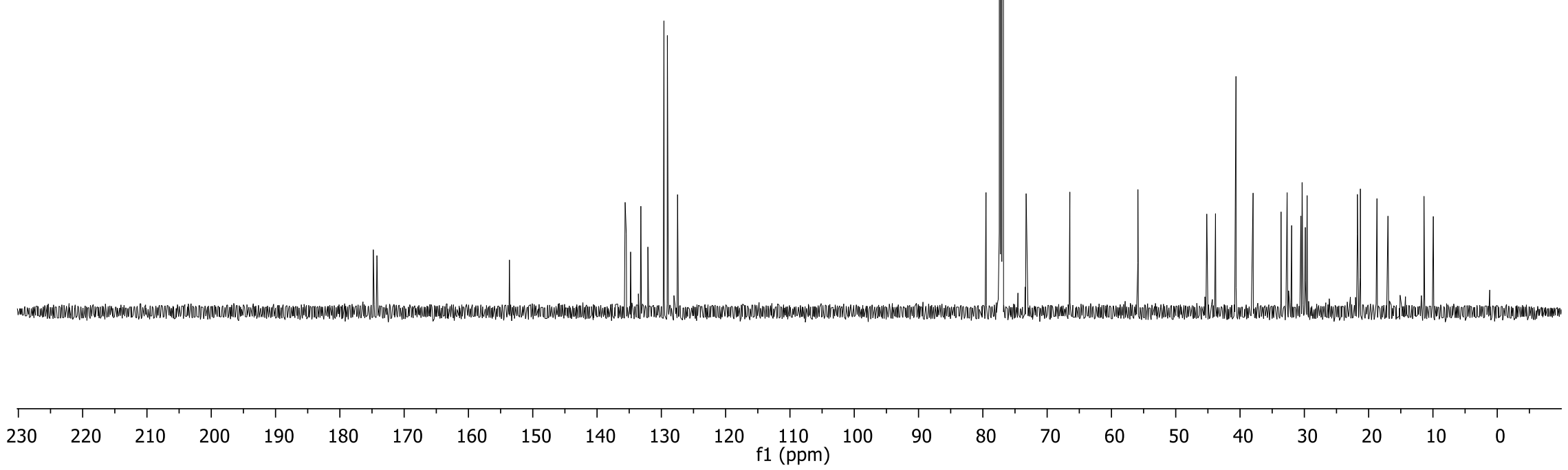




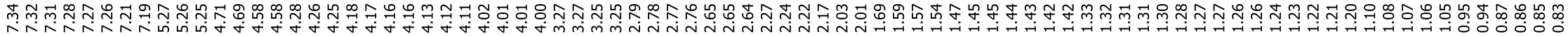

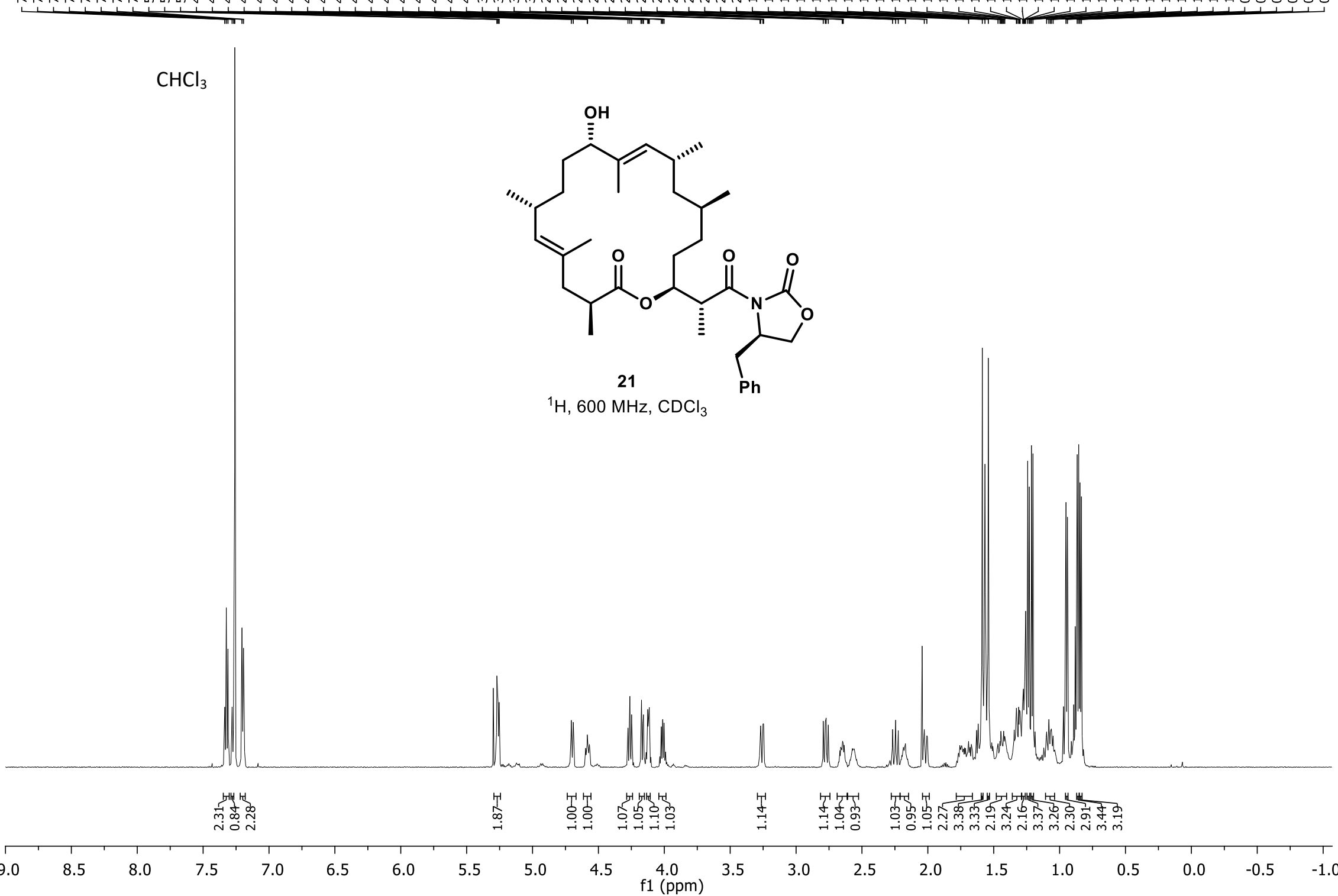




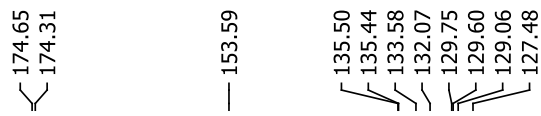

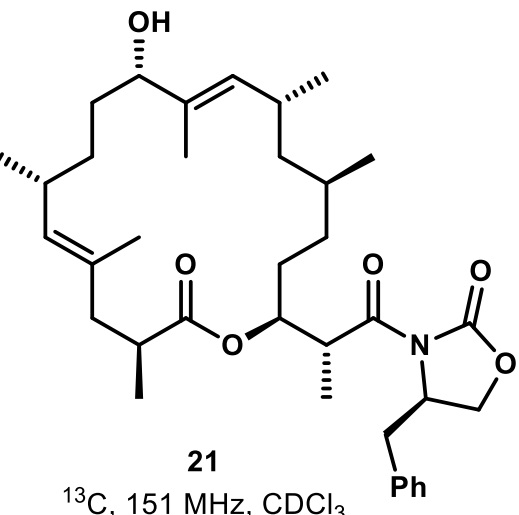

${ }^{13} \mathrm{C}, 151 \mathrm{MHz}, \mathrm{CDCl}_{3} \quad \mathrm{Ph}$

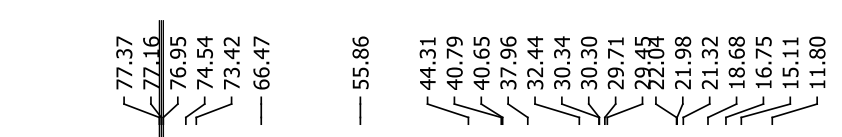

$\mathrm{CDCl}_{3}$

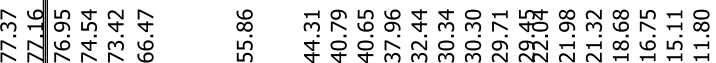

$\rightarrow>1$ 


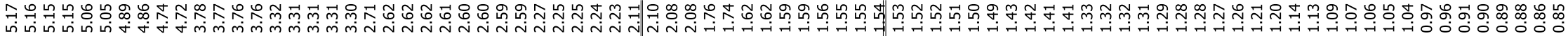

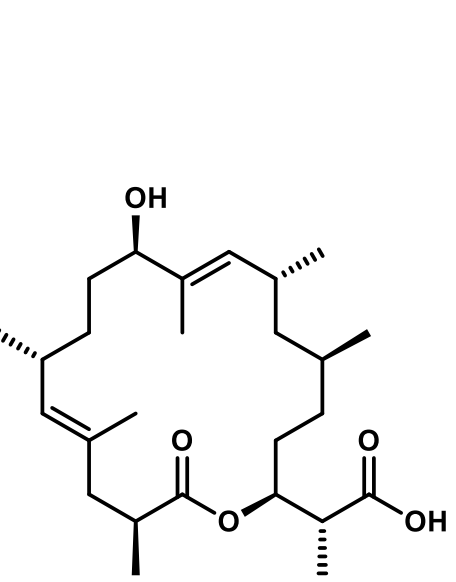

$$
1
$$

${ }^{1} \mathrm{H}, 600 \mathrm{MHz}, \mathrm{CD}_{3} \mathrm{OD}$

$\mathrm{CD}_{3} \mathrm{OH}$

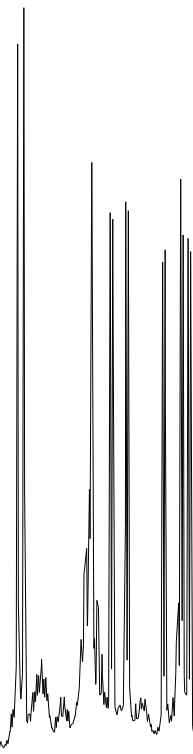

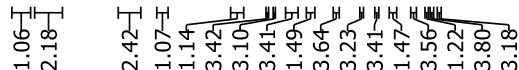

$4.0 \quad 3.5$

$\begin{array}{lll}2.5 & 2.0 & 1.5\end{array}$




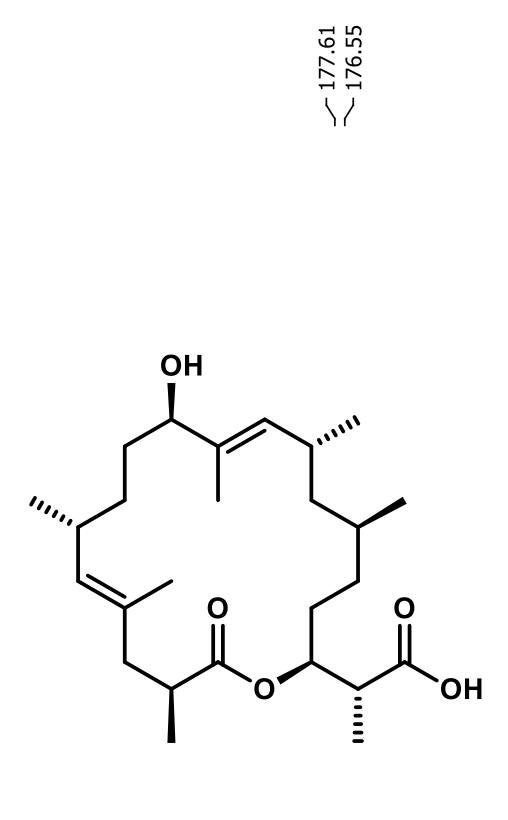

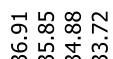

रोiा

음

क

$\mathrm{CD}_{3} \mathrm{OD}$

${ }^{13} \mathrm{C}, 151 \mathrm{MHz}, \mathrm{CD}_{3} \mathrm{OD}$
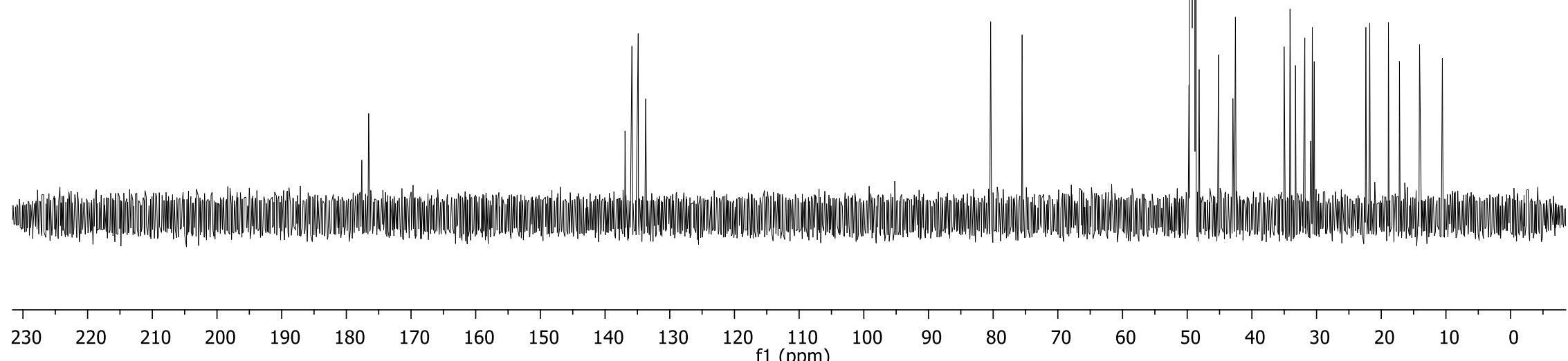


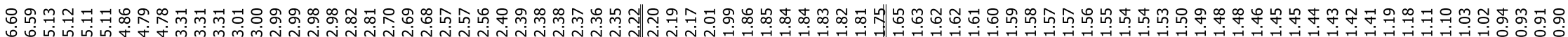

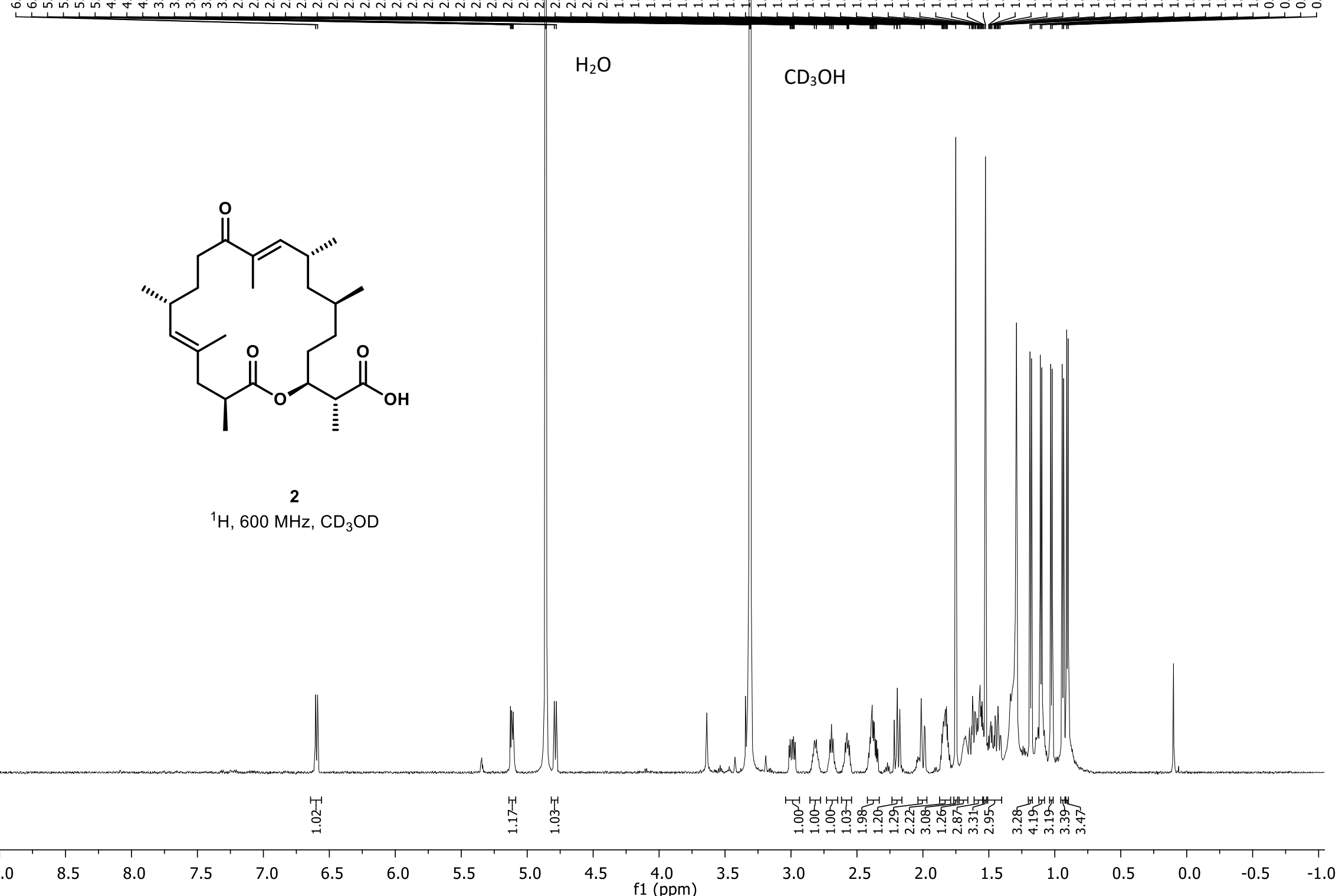




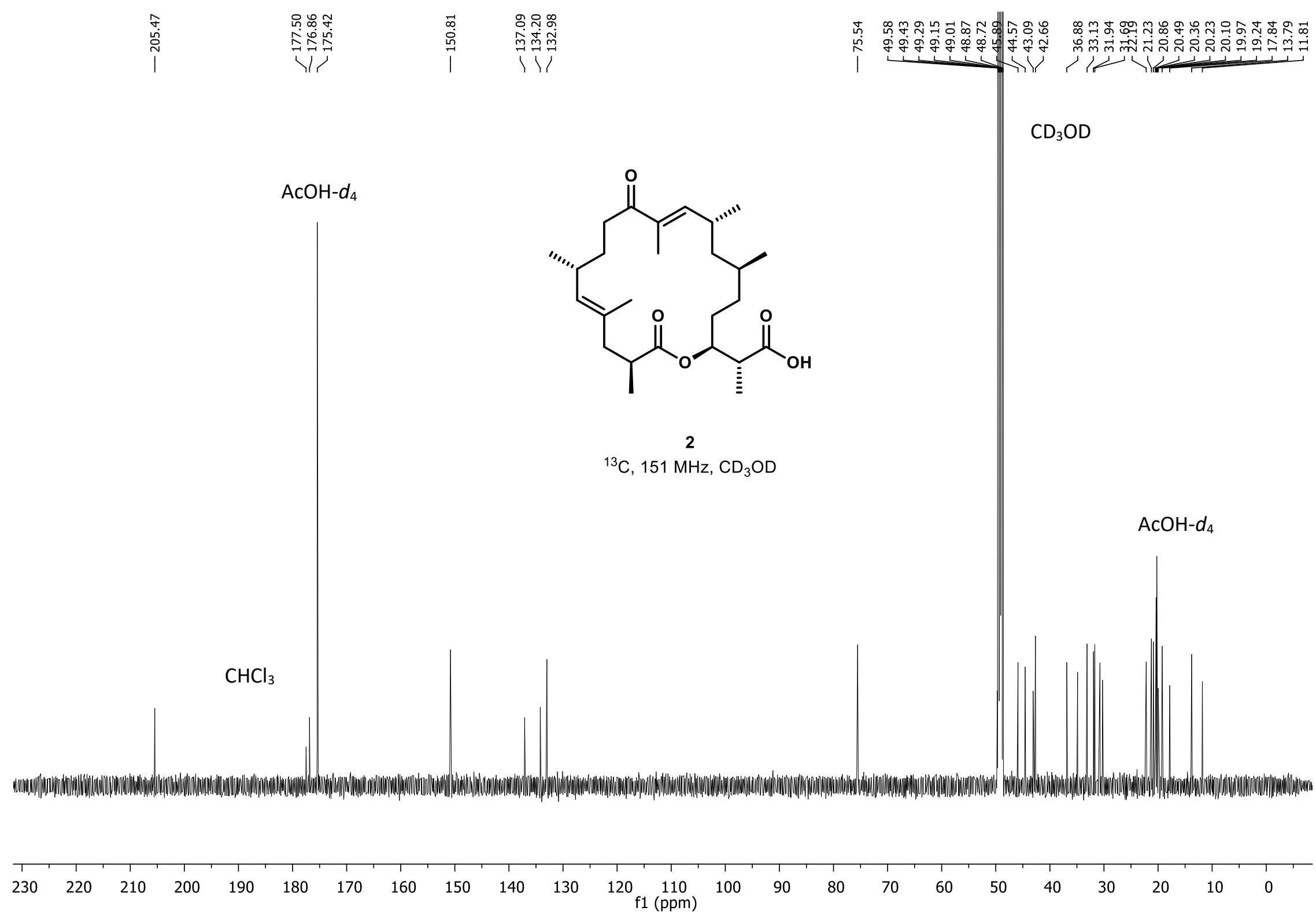

